\title{
Stochastic Optical Fluctuation Imaging - Labels and Applications
}

\author{
Dissertation \\ to acquire the doctoral degree in mathematics and natural science \\ "Doctor rerum naturalium" \\ at the Georg-August-Universität Göttingen \\ in the doctoral degree program GGNB \\ of the Georg-August University School of Science (GAUSS) \\ submitted by \\ Anja Huss \\ from Oberndorf a.N.
}

Göttingen, 2015 

Thesis Committee:

Prof. Dr. Jörg Enderlein (Referee)

Third Institute of Physics - Biophysics

Georg-August-University Göttingen

Prof. Dr. Fred S. Wouters (Co-Referee)

University Medical Center Göttingen

Georg-August-University Göttingen

Prof. Dr. Stefan W. Hell

Department of NanoBiophotonics

MPI for Biophysical Chemistry

Other Members of the Examination BoArd:

Prof. Dr. Sarah Köster

Institute for X-Ray Physics

Georg-August-University Göttingen

Dr. Alexander Egner, PD

Laser Laboratory Göttingen

Prof. Dr. Florentin Wörgötter

Third Institute of Physics - Biophysics

Georg-August-University Göttingen 


\section{Affidavit}

Hereby, I declare that the presented thesis has been written independently and with no other sources and aids than quoted.

Göttingen, 13.2.2015 


\section{Contents}

\begin{tabular}{ll}
\hline Abstract & 1
\end{tabular}

\begin{tabular}{lll}
\hline 1 & Introduction & 3
\end{tabular}

1.1 Fluorescence . . . . . . . . . . . . . . . . . . . . . . 3

1.2 Fluorescence microscopy $\ldots \ldots \ldots \ldots \ldots \ldots$

1.3 Resolution limit and point spread function $\ldots \ldots \ldots . \ldots$

1.4 Superresolution microscopy . . . . . . . . . . . . . . . . . . . 8

1.5 Neuronal signal transduction . . . . . . . . . . . . . . . . . . . 10

$\begin{array}{lll}2 & \text { Stochastic optical fluctuation imaging } & \mathbf{1 3}\end{array}$

$2.1 \quad$ Principle . . . . . . . . . . . . . . . . . . . . . . . . . 14

2.2 Computation and correction for bleaching . . . . . . . . . . 15

2.3 Effective pixel size . . . . . . . . . . . . . . . . 17

$2.4 \quad$ Fourier reweighting . . . . . . . . . . . . . . . . . . . . . . 18

2.5 Linearization of the intensity response $\ldots \ldots \ldots$. . . . . . . . 19

2.6 Fluorescence intermittency requirements . . . . . . . . . . . . 20

2.7 Setup requirements . . . . . . . . . . . . . . . . . . . 21

3 SOFI with Quantum Dots 23

3.1 Quantum Dots . . . . . . . . . . . . . . . . . . . . 23

3.2 Optimization of the imaging parameters $\ldots \ldots \ldots \ldots . \ldots . \ldots 24$

3.2.1 $\quad$ Exposure time optimization . . . . . . . . . . . . . . 24

3.2 .2 Frame number optimization . . . . . . . . . . . . . 24

3.2 .3 Drift reduction . . . . . . . . . . . . . . . 26

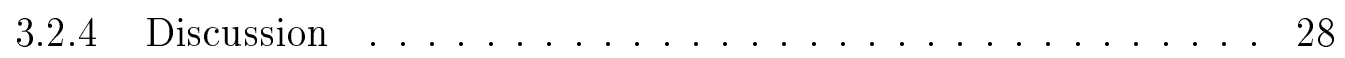

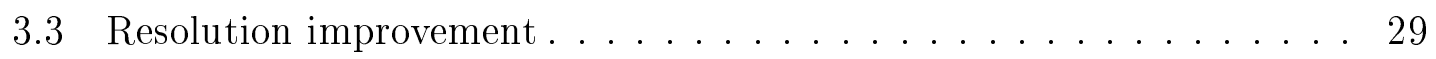

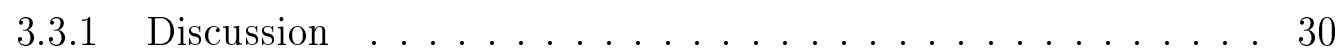

3.4 GABA $_{\mathrm{B}}$ receptor subunit trafficking $\ldots \ldots \ldots \ldots \ldots \ldots$

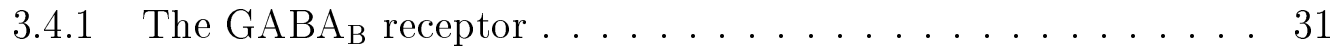

$3.4 .2 \quad 3 \mathrm{D}$, dual-color SOFI of GABA $\mathrm{B} 1$ and $\mathrm{R} 2 \ldots \ldots 33$

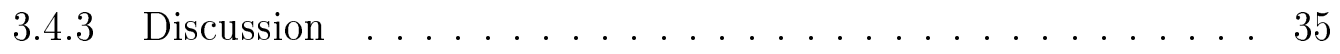

3.4 .4 Comparison of SOFI with confocal microscopy . . . . . . . . 35

3.4 .5 Discussion . . . . . . . . . . . . . . 36

3.5 Dependence of $\mathrm{Ca}^{2+}$ waves on distribution of ion channels . . . . . . 37

3.5 .1 The inositol trisphosphate and ryanodine receptors . . . . . . . 37 
$3.5 .23 \mathrm{D}$, dual-color SOFI of $\mathrm{IP}_{3} \mathrm{R} 1$ and RyR2 . . . . . . . . . . 37

3.5 .3 3D capability of SOFI: axial receptor distribution . . . . . . . 39

3.5 .4 Discussion . . . . . . . . . . . . . . . . 39

4 SOFI with reversibly photoswitchable fluorescent proteins 41

4.1 Reversibly photoswitchable fluorescent proteins . . . . . . . . . . . . . 41

4.2 Principle of photoswitching control . . . . . . . . . . . . . 45

4.2 .1 Inducing intensity fluctuations . . . . . . . . . . . . 45

4.2 .2 Ensemble studies of photoswitching kinetics . . . . . . . . . . . . 46

4.2 .3 Lifetimes determined from time traces . . . . . . . . . . . . . 49

4.2 .4 Influence of irradiation intensity on photoswitching . . . . . . 50

4.3 Determination of photoswitching . . . . . . . . . . . 51

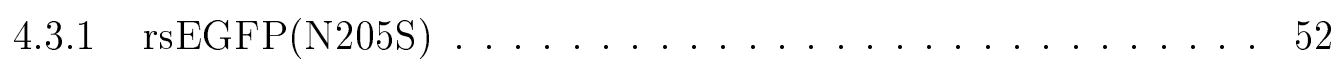

4.3 .2 Dreiklang . . . . . . . . . . . . . . . . . 54

4.3 .3 Discussion . . . . . . . . . . . . . . . . 54

4.4 Calibration of photoswitching . . . . . . . . . . . 57

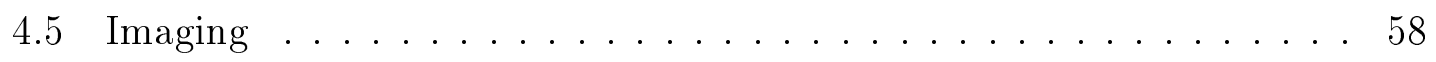

4.5 .1 Continuous excitation . . . . . . . . . . . . . 58

4.5 .2 Pulsed excitation . . . . . . . . . . . . . . . . 62

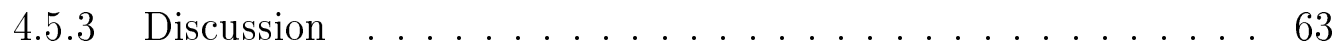

\begin{tabular}{|lll}
\hline 5 & SOFI with organic dyes & 67
\end{tabular}

5.1 Organic dyes . . . . . . . . . . . . . . . . . 67

5.2 dSTORM: (direct) stochastic optical reconstruction microscopy . . . . 67

5.3 Organization of the axon initial segment . . . . . . . . . . . . . 69

5.4 Blinking flexibility . . . . . . . . . . . . . . . 70

$5.4 .1 \quad$ Spectrin with Alexa Fluor 647: photoswitching optimized for STORM . . . . . . . . . . . . . . . 71

5.4 .2 Spectrin with Alexa Fluor 647: photoswitching optimized for SOFI . . . . . . . . . . . . . . . . 73

5.4 .3 Sodium channels with Alexa Fluor $488 \ldots \ldots$. . . . . . 76

5.4 .4 Discussion . . . . . . . . . . . . 77

\begin{tabular}{|lrl}
\hline 6 & Conclusions and outlook & 81
\end{tabular}

$\begin{array}{lll}7 & \text { Setups and protocols } & 85\end{array}$

7.1 Sample preparation and imaging conditions . . . . . . . . . . 85 
7.1.1 GABA $_{\mathrm{B}}$ receptor immunostaining . . . . . . . . . . 85

$7.1 .2 \quad \mathrm{IP}_{3}$ and ryanodine receptor immunostaining $\ldots . . . . .85$

7.1.3 SOFI with Quantum Dots: imaging conditions . . . . . . . . 86

7.1.4 Spectrin and $\mathrm{Na}_{\mathrm{V}}$ immunostaining . . . . . . . . . . . 86

7.1 .5 Switching buffer . . . . . . . . . . . . . . . 87

7.1 .6 SOFI with organic dyes: imaging conditions . . . . . . . . 87

7.1 .7 Reversibly photoswitchable fluorescent proteins . . . . . . . . 87

7.1 .8 SOFI with RSFPs: imaging conditions . . . . . . . . . . 89

7.2 Setups . . . . . . . . . . . . . . . . . . . . 89

7.2 .1 Widefield setup equipped with LEDs . . . . . . . . . . . . 89

7.2.2 Setup for total internal fluorescence (TIRF), widefield, and confocal spinning disk imaging . . . . . . . . . . . 89

7.2 .3 Laser pulse generation . . . . . . . . . . . . . . . 9 90

7.2 .4 Surface power densities . . . . . . . . . . . . . . . 91

7.3 Algorithms . . . . . . . . . . . . . . . . . . . 91

\begin{tabular}{lll}
\hline & Supplementary data & 93
\end{tabular}

$8.1 \quad$ Determination of photoswitching $\ldots \ldots \ldots \ldots . \ldots . \ldots . \ldots 93$

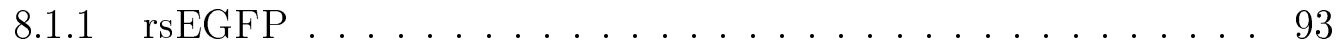

8.1 .2 rsEGFP2 $\ldots \ldots \ldots \ldots \ldots \ldots \ldots \ldots \ldots$

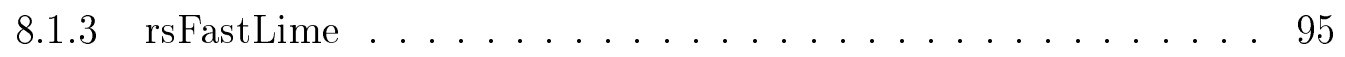

$8.1 .4 \quad \operatorname{Dronpa}(\mathrm{M} 159 \mathrm{~T}) \ldots \ldots \ldots \ldots \ldots$

$8.1 .5 \quad$ Discussion $\ldots \ldots \ldots \ldots \ldots \ldots \ldots \ldots$

\begin{tabular}{lr}
\hline Appendix & 99
\end{tabular}

Bibliography . . . . . . . . . . . . . . . . . . . . . . 99

List of Figures . . . . . . . . . . . . . . . . . . . . . . . . 108

List of Tables . . . . . . . . . . . . . . . . . . . . . . . 109

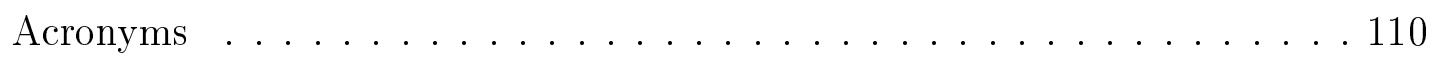

Acknowledgments . . . . . . . . . . . . . . . . . . . . . 113 


\section{Abstract}

Superresolution fluorescence imaging is an important tool for studying subcellular structures and processes. A technique with great potential is stochastic optical fluctuation imaging (SOFI), a fluorescence imaging modality that yields superresolved spatial resolution, 3D-sectioning and high image contrast by making use of independent temporal intensity fluctuations (blinking) of fluorescent labels. The compatibility of SOFI with a large range of blinking kinetics allows for the use of a great variety of labels. The inherent blinking of Quantum Dots can be utilized as well as the induced intensity fluctuations of organic dyes and fluorescent proteins, allowing for superresolution imaging in a multitude of systems.

Exploiting the natural blinking of Quantum Dots for SOFI is straightforward and requires merely a basic widefield microscope and low excitation intensities. The provided optical sectioning and resolution increase are highly useful for biological applications, for example enabling the quantification of the distribution of calcium channels in the neuronal ER membrane in order to link ER structure to properties of calcium waves.

Studying the organization of live cells with SOFI is enabled by reversibly switchable fluorescent proteins (RSFPs). RSFPs are a type of fluorescent proteins whose absorption and emission properties depend strongly on illumination. Light of certain wavelengths stimulates transitions between fluorescent and non-fluorescent states, thus opening up the possibility to induce statistically independent temporal intensity fluctuations. The dependence of the photoswitching kinetics on the irradiation intensities of several RSFPs was studied, and Dreiklang and rsEGFP(N205S) were identified as suitable labels. After confirming the lifetimes of the fluorescent and nonfluorescent states with calibration measurements, the induced blinking was used for SOFI on live mammalian cells.

SOFI is much more robust regarding intensity fluctuation kinetics than localization methods such as dSTORM. As a result, SOFI can be applied in cases where the blinking properties render single molecule localization impossible. This is illustrated by comparing SOFI and STORM results of studies of the organization of the axon initial segment.

SOFI is highly suitable for densely labeled structures as well as for samples with only sparse labeling, and for all kinds of blinking kinetics. Superresolved images can be acquired at low excitation power, limiting photodamage of the sample and the fluorophores even for prolonged data acquisition. Combined with its 3D-sectioning 
capability, this allows for the recording of z-stacks of superresolved images over large axial distances on a modest widefield setup. Due to its straightforward and robust nature, SOFI is therefore advantageous for a variety of applications. 


\section{Introduction}

\section{$1.1 \quad$ Fluorescence}

The phenomenon of light emission due to radiative relaxation from an electronic excited state is generally termed luminescence. It takes on different forms and occurs in many different compounds, so called fluorophores.

The energy levels and possible transitions of a fluorescent organic compound are illustrated by a Jablonski diagram (Fig. 1). The singlet ground state $\mathrm{S}_{0}$ and the excited states (first, second etc. electronic states $\mathrm{S}_{1}, \mathrm{~S}_{2}$ etc.) of an organic fluorophore each contain several vibrational levels 1 . The spin-allowed radiative relaxation from an electronic excited state to the ground state is called fluorescence. Usually, the process starts with excitation of an electron from $S_{0}$ to one of the vibrational levels of $\mathrm{S}_{1}$, which is followed by non-radiative relaxation to the vibrational ground state of $\mathrm{S}_{1}$. This internal conversion (IC) takes $\sim 10^{-12} \mathrm{~s}$. Subsequently, the system relaxes to a higher vibrational level of $S_{0}$ under light emission, and further to the vibrational ground level of $\mathrm{S}_{0}$ by IC. With fluorescence lifetimes of $\sim 10 \mathrm{~ns}\left(10^{-8} \mathrm{~s}\right)$, radiative relaxation is considerably slower than non-radiative vibrational relaxation. Due to the additional non-radiative vibrational relaxations, not all energy absorbed by the fluorophore is emitted again. Therefore, light emitted by a fluorophore is red shifted relative to the exciting light, causing a difference between absorption and emission spectra known as Stokes shift.

Alternatively, intersystem crossing (ISC) from the first singlet state to the first triplet state $T_{1}$ can occur. After this spin-forbidden transition, the system relaxes to $\mathrm{S}_{0}$ in a process called phosphorescence. Due to the likewise spin-forbidden nature of the relaxation, the lifetimes of dark triplet states are orders of magnitude longer than those of singlet states, typically milliseconds to seconds. $T_{1}$ is energetically lower than $\mathrm{S}_{1}$, therefore light of longer wavelength is emitted due to relaxation from $\mathrm{T}_{1}$ compared to $\mathrm{S}_{1}$.

Organic dyes are a well established class of fluorophores. They are aromatic organic molecules with HOMO-LUMO (highest occupied molecular orbital and lowest unoccupied molecular orbital) gaps in the energy range of visible light. Regarding the electronic transitions, the HOMO corresponds to the electronic ground state and the LUMO to the first excited state. As a general rule, a molecule with a more extended $\pi$-electron-system has a smaller HOMO-LUMO gap and thus a more red-shifted spectrum than a molecule with a smaller $\pi$-system. For fluorescence 


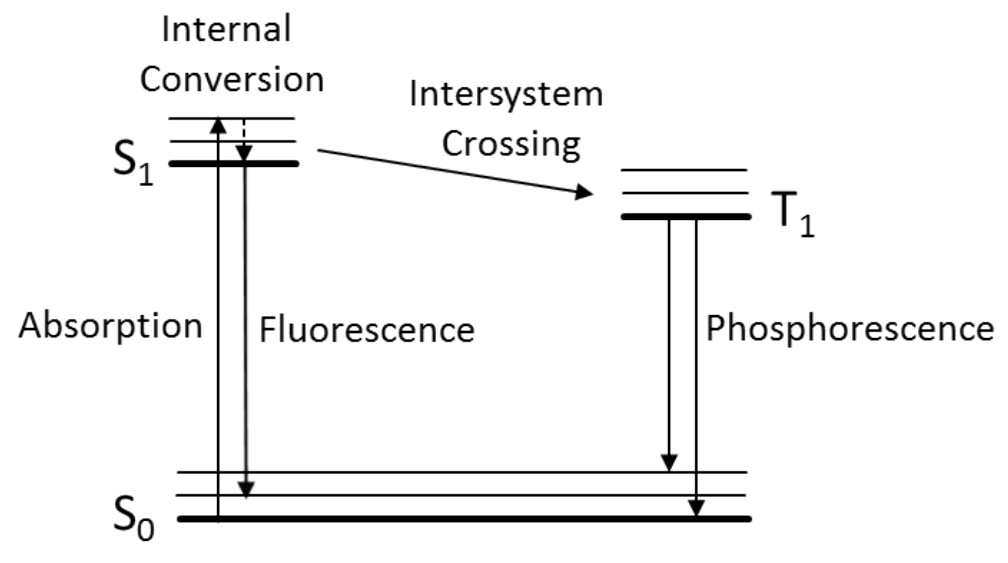

Figure 1. Energy states and transitions in a fluorescent molecule illustrated by a Jablonski diagram. Excitation from the ground state $S_{0}$ to a vibrational level of the first excited state $S_{1}$ occurs by absorption of light of a suitable wavelength. The system relaxes to the vibrational ground state of $S_{1}$ via internal conversion, followed by relaxation to a vibrational level of $S_{0}$ under fluorescence and non-radiative relaxation to the vibrational ground level of $S_{0}$. Phosphorescence is the radiative relaxation from $T_{1}$ to $S_{0}$ under spin conversion and occurs after intersystem crossing.

microscopy, organic dyes are attached to the structures of interest with antibodies in a process called immunostaining. Fluorescent proteins (FPs) are a related class of emitters. They are genetically encoded fluorophores which can be fused to any protein by means of molecular biology, allowing for cells expressing fluorescent labels linked to the structures of interest. The chromophore, the specific part of the fluorophore responsible for luminescence, is an aromatic system formed from amino acids. It is enclosed in a protein hull, the $\beta$-barrel. Quantum dots (QDots), in contrast, are a fundamentally different type of fluorophore. These small semi-conductor crystals exhibit fluorescence due to quantum confinement. Like organic dyes, they are often used in immunostaining protocols.

\subsection{Fluorescence microscopy}

Fluorescence microscopy is an immensely important technique for studying cellular structures and processes using fluorophores as labels. Very generally, excitation light is focused into the sample by a system of lenses. The resulting fluorescence light is then detected either by eye or using a camera. The Stokes shift allows for the separation of the fluorescence light from the excitation light using optical filters before detection. An example of a fluorescence image is shown in Fig. 2. To eluci- 
date the relative positions of different parts of a cell, three different structures of a cultured neuron were fluorescently labeled. The fluorophores were chosen to allow for separate detection of all emission wavelengths and subsequent merging into one triple-color image. In the merged image, the nucleus is depicted in blue, the somatodendritic region in red and the axon initial segment in green. With fluorescence microscopy and targeted labeling it is thus possible to localize the nucleus, distinguish between the axon and the dendrites and determine the extent of the axon initial segment.

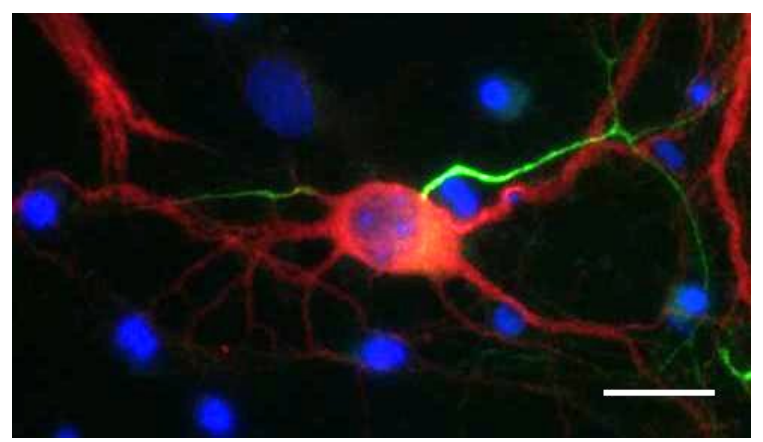

Figure 2. Widefield fluorescence image of nucleus (blue, DAPI), somatodendritic region (red, MAP2) and axon initial segment (green, PanNaV) of a neuron. Scale bar: $20 \mu \mathrm{m}$

In conventional widefield microscopy, the whole sample is irradiated and light emitted from labels within and away from the focal plane is detected. A drawback of this approach is its poor optical sectioning capability. In order to obtain a clear view of the structures in only a narrow slice of the sample, fluorescence light from other areas must be excluded from detection.

Confocal microscopy discards out-of-focus light by focusing the fluorescence light on a pinhole which only light from a small volume in the sample can pass. To obtain a complete image, the sample is scanned laterally and the image is constructed point by point. Axially, the fluorescence stemming from out of focus areas is prevented from reaching the detector 2 . However, the scanning speed is limited. Furthermore, the signal-to-noise-ratio (SNR) often is low due to the detection of only a small part of the sample fluorescence. Accumulating several scans per point or increasing the detection time per point improves the SNR, but increases the imaging time as well. A high SNR can also potentially be generated using higher powers of the excitation light, but this also increases the risk of photobleaching.

For improved scanning speed and SNR, a confocal spinning disk microscope uses several focal spots of excitation light at a time 3 . Commercial spinning disk units contain two disks that spin synchronously between the light source and the 
objective. The first disk contains an array of 20,000 spirally arranged microlenses which collect the excitation light and focus it through the second disk with pinholes, called the Nipkow disk, in the form of individual beams. The divergent beams from each pinhole then are focused on the back aperture of the objective when they pass through the microscope's tube lens. In this manner, the system creates multiple focal spots moving over the sample. Since the light emitted from the sample upon excitation has to pass the pinhole array of the Nipkow disk in order to reach the detector, only fluorescence light from the confocal volumes of the multiple focal spots is detected. To improve the collection efficiency, the dichroic mirror is placed between the two disks and the light through the pinhole array is focused directly on the detector, thus avoiding the passage through the microlens array. Consequently, the spinning disk system offers confocal scanning over the sample with several hundreds of spots simultaneously, thereby increasing the imaging speed and reducing the risk of photobleaching.

\subsection{Resolution limit and point spread function}

The general drawback to fluorescence microscopy is that the maximum achievable resolution is limited by fundamental properties of light. For light of wavelength $\lambda$, a refractive index $n$ of the medium, and the half-angle $\theta$ of the maximum cone of light that can enter or exit the lens, the smallest possible lateral distance $d$ of two resolvable emitters is the diffraction limit

$$
d=\frac{\lambda}{2 n \sin \theta}
$$

where $n \sin \theta=\mathrm{NA}$ is the numerical aperture of the objective lens. This first definition for the resolution limit was developed by Ernst Abbe 5 . Signals closer than $d$ cannot be separated. For example, labels emitting at a wavelength of $500 \mathrm{~nm}$, imaged using an objective lens of $\mathrm{NA}=1.4$, under the condition of perfect optics and imaging conditions, are just separable at a distance of around $180 \mathrm{~nm}$. Emitters closer together cannot be differentiated with conventional fluorescence microscopy.

To measure the resolution of an optical system, the full width at the half maximum of the intensity (FWHM) of the point spread function (PSF) is a more convenient criterion. The PSF is the response of an imaging system to a point source. It typically has the shape of an Airy disk but can be approximated as a Gaussian function. When imaging a point source, the detected fluorescence is a convolution of the original point source with the PSF of the system. Therefore, resolving signals is 


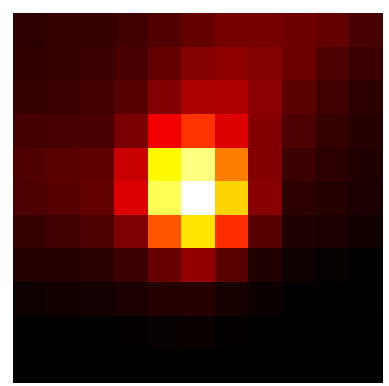

(a) Widefield image of PSF

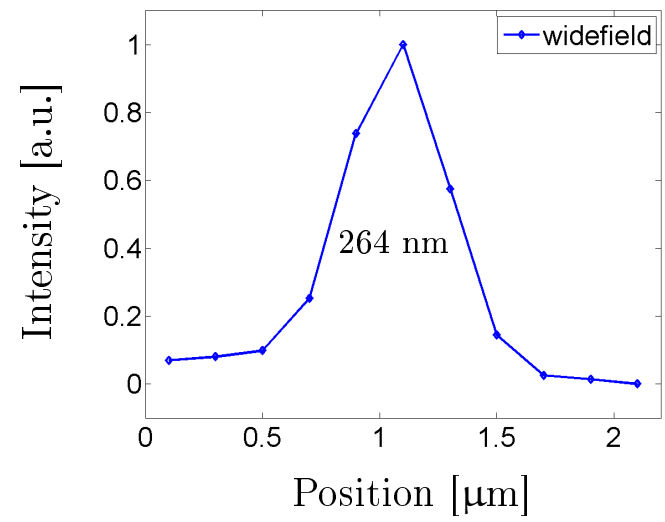

(b) Profile in $\mathrm{x}$-direction

Figure 3. Widefield image (a) and profile in $\mathrm{x}$-direction (b) of a single $Q D o t$ with emission wavelength $\lambda_{e m}=525 \mathrm{~nm}$. Effective pixel size $=100 \mathrm{~nm}$, measured $F W H M=264 \mathrm{~nm}$.

possible to a smaller distance when the system has a narrow PSF. The narrower the PSF, the better resolved are the structural details of an imaged sample, for example a cell. The theoretical lateral FWHM (in the sample plane) is given by

$$
\mathrm{FWHM}_{x, y}=\frac{0.51 \lambda}{\mathrm{NA}} \stackrel{2}{ },
$$

while the axial FWHM (along the optical axis) amounts to

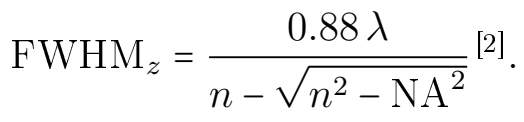

According to Eq. 2, the theoretical lateral FWHM, for NA = 1.4, for the QDots used to label neuronal neurotransmitter receptors (see section 3) are $191 \mathrm{~nm}$ for the QDots with an emission wavelength of $\lambda_{e m}=525 \mathrm{~nm}\left(\mathrm{QD}_{525}\right)$, and $220 \mathrm{~nm}$ for $\lambda_{e m}=605 \mathrm{~nm}\left(\mathrm{QD}_{605}\right)$. The PSF is typically measured using a sample of bright emitters smaller than the resolution limit, like dye-filled spheres or QDots. Fig. 3 shows the image of an individual QDot with $\lambda_{e m}=525 \mathrm{~nm}$, measured on a widefield setup equipped with LEDs. The $\mathrm{FWHM}_{x, y}$ was determined by Gaussian fitting of ten emitters each to be on average $264 \mathrm{~nm}( \pm 14 \mathrm{~nm})$ for $\mathrm{QD}_{525}$ and $296 \mathrm{~nm}( \pm 20$ $\mathrm{nm})$ for $\mathrm{QD}_{605}$. The discrepancy to the theoretical values is due to imperfections in the optical system.

A related difficulty for fluorescence imaging is the lower achievable limit of the focal spot size. Due to diffraction, light cannot be focused into a spot of arbitrarily 
small size. Instead, the focal spot takes the shape of an Airy disk characterized by the wavelength of the focused light and the size of the aperture. As a result, when excitation light is focused into a sample with fluorescent markers, many emitters are excited at once and are indistinguishable in detection.

The reason for these effects lies in the limitation of optical systems to transmit high spatial frequencies of the optical transfer function (OTF), the Fourier transform of the PSF. An optical system acts as a low pass filter, blocking the transmission of the highest spatial frequencies and ensuring that the OTF always has a finite support. The larger the support of the OTF, the more spatial frequencies are transmitted, leading to a narrower PSF and a higher resolution. If all spatial frequencies that are contained in the emission of a fluorophore were transmitted through the optical system, the exact location of the fluorophore could be detected, instead of a pattern covering an area much larger than the emitter.

\subsection{Superresolution microscopy}

Structures smaller than the diffraction limit as well as cellular processes unobservable at conventional resolution are of great interest for biological research. In the last two decades, techniques to overcome this barrier have been developed. These superresolution microscopy techniques allow for new insights into systems that previously were impossible to study.

The first technique to break the diffraction barrier was stimulated-emissiondepletion (STED) fluorescence scanning microscopy 6. Similar to conventional confocal microscopy, a sample is scanned by a focused laser and the image is constructed point by point. The resolution is limited by the fact that all fluorophores in the focal spot are transferred to their excited state, and the resulting spontaneous emission can not be assigned to the location of the fluorophore that gave rise to it. Therefore, structures smaller than the focal spot size cannot be resolved. To overcome this problem, an additional laser beam stimulates emission of the fluorophores in the outer rim of the focal spot. This STED beam hence depletes the excited state in the area it irradiates, suppressing further spontaneous emission. Fluorescence consequently only stems from the central part of the focal spot, where no depletion took place. The achievable resolution mainly depends on the intensity of the STED beam. The higher the intensity is, the smaller is the area containing excited fluorophores and the higher is the resolution. Thus, resolutions in the nanometer range have been shown ${ }^{7}$. The concept was extended to other reversible saturable optical 
fluorescence transitions (RESOLFT) ${ }^{8}$ of labels, such as fluorescent and dark states of fluorescent proteins. These transitions require considerably less energy than the saturation of the excited state, reducing photobleaching and other light-induced damaging.

For structured illumination microscopy (SIM) [9, a known illumination pattern is moved over the sample, the fluorescence image at each pattern position is recorded and the superresolved image is subsequently calculated from the individual frames. By using a known pattern of transmittable frequency for excitation, normally nontransmittable higher spatial frequencies carrying additional information can be observed. The product of the excitation pattern and the pattern of the high spatial frequencies are moiré fringes of low frequency that can be transmitted and detected. Thus, an image with up to doubled resolution and additional optical sectioning can be reconstructed from a set of images recorded with different excitation patterns. Conveniently, conventional dyes can be used without modification. A related technique is image scanning microscopy (ISM) 10, which uses a laser focus as a special type of structured illumination. The fluorescence elicited by the laser at each point is imaged on a position-sensitive camera, and an image with up to doubled resolution can be calculated. Confocal setups as well as the faster confocal spinning disk setups can be adapted for ISM 11 .

Another important group of superresolution methods is based on the localization of single emitters. These localization methods rely on the temporal separation of the emission of individual fluorophores. As mentioned earlier, the response of a microscope to a fluorophore is the point spread function (PSF). Given sufficient information about the imaging system, the emitter's location can be calculated from the detected PSF pattern. This is possible with high precision for individual patterns, but not when the patterns of several emitters overlap. Naturally, the response of an imaging system to a densely labeled biological sample containing many fluorophores is an image with many overlapping PSF patterns. Consequently, structures smaller than the diffraction limit are not resolved. To achieve superresolution by localizing individual fluorophores for such structures, it is necessary to separate their fluorescence in time. This is achieved by allowing only a small subset to emit light at any time. The core idea is to record a movie of emitters exhibiting fluorescence intermittency (blinking). In each movie frame, a small number of emitters fluoresce, while the majority remains in a long-lived dark state. The recorded fluorescence patterns thus stem from a small subset distributed over the whole sample area. If the subset is small enough, the patterns do not overlap and the emitters can be localized. 
In the next frame, different emitters are localized. This process is repeated until a complete superresolved image can be reconstructed. Different methods to obtain the necessary long-lived dark states of the labels were adapted for single-molecule localization techniques. Stochastic optical reconstruction microscopy (STORM) 12] relies on a combination of two fluorophores as one probe to generate the required short fluorescent and long-lived dark state. Photoactivated localization microscopy (PALM) 13 and fluorescence photoactivation localization microscopy (FPALM) 14] use the photoactivation and subsequent bleaching of photoactivatable fluorescent proteins (PA-FPs). A further development that is widely used nowadays is directSTORM (dSTORM) 15. Here, only one organic dye is used to label one protein of interest, and the blinking is controlled using a switching buffer.

Stochastic optical fluctuation imaging (SOFI) 16[17 makes use of intensity fluctuations of emitters as well, but in contrast to the localization methods, the increased resolution is obtained directly from an analysis of the independent temporal fluctuations.

\subsection{Neuronal signal transduction}

The nervous system including the brain is composed of a dense network of connected neurons. Neurons consist of three functionally distinct parts, the soma and two types of processes, axons and dendrites. Generally speaking, the typically long and branched axon is responsible for the output signals, while the dendrites are the processes receiving the input signals $\frac{18}{18}$. These signals are short electrical pulses called action potentials (APs). An AP can be described as an electric potential wave that propagates due to opening and closing of ion channels, temporally allowing for certain ions to pass through the membrane and changing the membrane potential 19. At the synapses, the contact points between neurons, information is typically conveyed by a variety of chemical compounds. The release of these neurotransmitters is triggered by the AP on the presynaptic site (the output neuron). Subsequently, neurotransmitters are detected by specialized receptors in the dendritic membrane of the postsynaptic neuron (the input neuron) and trigger further signaling by opening of ion channels. Neurotransmitter receptors play a key role in synaptic plasticity, the modification of synaptic efficacy, and therefore in memory acquisition and information storage 20 .

Neuronal signal transduction thus depends on many parameters, such as the location and behavior of different types of ion channels, the affinity of ligand-gated 
channels for their ligands, the availability of ligands, the transportation of membrane proteins from the soma and their availability. Due to their importance and the disastrous effects their loss or even impaired functionality have on health, neurons are the focus of a multitude of research approaches. 


\section{Stochastic optical fluctuation imaging}

Stochastic optical fluctuation microscopy (SOFI) gains additional information by analysis of the stochastic fluctuation intermittency of the fluorescent labels. From the blinking, an image with increased resolution is calculated, as illustrated in Fig. 4.

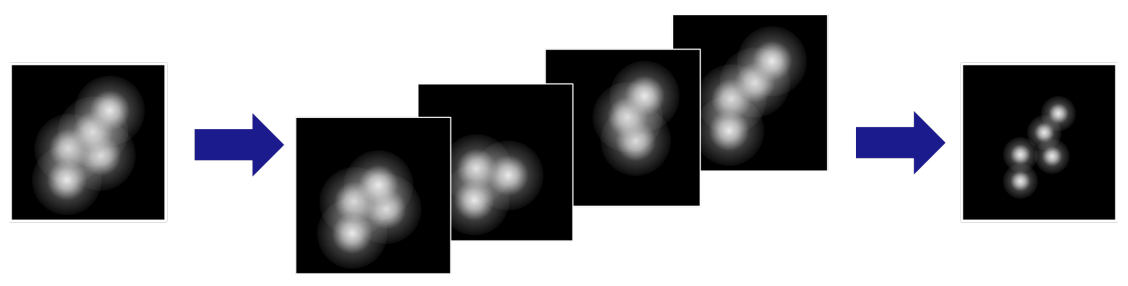

Figure 4. Schematic of the SOFI process. On a widefield setup, a movie of a sample labeled with blinking emitters is recorded. In each movie frame, different fluorophores are emitting, causing different intensity fluctuations on each pixel of the camera chip. From these temporal intensity fluctuations, a SOFI image with increased resolution and suppressed background is calculated.

In order to obtain a superresolved image, the emissions of individual emitters must be disentangled. For SOFI, this disentangling is based on uncorrelated but homogeneous intensity fluctuations $s(t)$ of the emitters. To generate a SOFI image, a movie of a suitably labeled sample is recorded and time traces of the intensities recorded by all pixels are calculated. Then, for the most basic approach, the variance $\operatorname{Var}(\mathrm{r})=\left\langle\left(s(t)-\langle s\rangle_{t}\right)^{2}\right\rangle_{t}$ of the intensity fluctuation is analyzed (with angular brackets denoting averaging over time $t$ ). For our case of independent blinking without correlation between the intensity fluctuations of different emitters, the variance is non-zero only for fluorescence signals from the same emitter. Consequently, the variance of the sum of the blinking of several emitters is the sum of the variances of the individual fluctuations of each emitter; the emitters contribute individually to the variance. If fluorescence from several emitters is recorded on a pixel, the overall variance of the recorded intensity signal therefore is the sum of the individual variances of the emitters.

For a fluctuating fluorescence signal $s(t)$ multiplied with a spatial profile $U(r)$, the variance is the variance of the fluctuations multiplied with the square of the spatial profile:

$$
\begin{aligned}
\operatorname{Var}(r) & =\left\langle\left(U(r) \cdot s(t)-\langle U(r) \cdot s\rangle_{t}\right)^{2}\right\rangle_{t} \\
& =U^{2}(r) \cdot\left\langle\left(s(t)-\langle s\rangle_{t}\right)^{2}\right\rangle
\end{aligned}
$$


This corresponds to a narrowing of the profile. Since the emitters contribute individually to the variance, the spatial profiles of all emitters in a sample shrink independently, even if they overlap strongly in the recorded movie. This corresponds to a shrinking of the microscope's PSF, and therefore to a resolution increase. By calculating the variance of intensity fluctuations, fluorescence emissions of different emitters can thus be disentangled and a resolution increase is achieved.

This concept can be generalized to higher SOFI orders $n$. While analyzing the variance of the fluctuations gives rise to a second order SOFI image, higher order cumulants are used for third, fourth etc. order SOFI, corresponding to a further shrinking of the PSF. Furthermore, correlating signals at different times by introducing a time lag $\tau$ eliminates short correlation noise from any SOFI order. For example, this is achieved for second order SOFI by calculating the covariance between times $t$ and $t+\tau$ according to $\operatorname{CoVar}(r)=\left\langle\left(s(t)-\langle s\rangle_{t}\right) \cdot\left(s(t+\tau)-\langle s\rangle_{t}\right)\right\rangle_{t}$.

\subsection{Principle}

The intensity distribution of a widefield image of a sample labeled with $N$ emitters is described by

$$
F(\mathbf{r}, t)=\sum_{j=1}^{N} U\left(\mathbf{r}-\mathbf{r}_{j}^{\prime}\right) \epsilon_{j} s_{j}(t)
$$

$U\left(\mathbf{r}-\mathbf{r}^{\prime}\right)$ is the point spread function of the microscope, describing the intensity recorded at position $\mathbf{r}$ of the image for an emitter of unit brightness at position $\mathbf{r}^{\prime} . \epsilon_{j}$ is the maximum brightness of the $j^{\text {th }}$ molecule with $1 \leq j \leq N$, and $s_{j}(t)$ describes the time-dependent brightness of the emitter. Since the blinking of different emitters is statistically independent, the temporal second order cumulant of the signals emitted by two fluorophores is zero. This is expressed by

$$
\begin{aligned}
C_{2}\left[s_{j}(t), s_{k}(t+\tau)\right] & \equiv\left\langle\delta s_{j}(t) \cdot \delta s_{k}(t+\tau)\right\rangle \\
& =\delta_{j k} \epsilon_{j}^{2} f(\tau) .
\end{aligned}
$$

$\delta s_{j}(t)=s_{j}(t)-\left\langle s_{j}\right\rangle$ is the temporal brightness variance of the $j^{\text {th }}$ molecule, Kronecker's symbol $\delta_{j k}$ is one for equal indices and zero for differing indices, $f(\tau)$ is a function describing the temporal correlation of the brightness of one molecule, and angular brackets denote averaging over time $t$. Therefore, the temporal second order cumulant is zero for two input signals $s_{j}(t)$ and $s_{k}(t)$ fluctuating in a statistically 
independent manner. This can also be generalized to more than two input sources by using third, forth etc. order cumulants.

For SOFI analysis, a movie consisting of a number of images $F(\mathbf{r}, t)$ (see Eq. 4) is recorded. To calculate the corresponding SOFI image, the $n^{\text {th }}$ order cumulants are calculated and integrated over the time lags $\tau_{m}$. This leads to the following equation for second order SOFI:

$$
S_{2}(\mathbf{r})=\int d \tau_{1} C_{2}\left[F(\mathbf{r}, t), F\left(\mathbf{r}, t+\tau_{1}\right)\right],
$$

with $F(\mathbf{r}, t)$ being the image at time $t$ and $F\left(\mathbf{r}, t+\tau_{1}\right)$ the image at time $t+\tau_{1}$. Introducing the intensity distributions of the images according to Eq. 4 leads to a double sum over products of $\left\langle\delta s_{j}(t) \delta s_{k}\left(t+\tau_{1}\right)\right\rangle$. However, cross terms with $j \neq k$ cancel out, therefore the SOFI image is described by the single sum

$$
S_{2}(\mathbf{r})=\left(\int d \tau_{1} f\left(\tau_{1}\right)\right) \sum_{j=1}^{N} U^{2}\left(\mathbf{r}-\mathbf{r}_{j}^{\prime}\right) \epsilon_{j}^{2},
$$

assuming identical blinking statistics for all fluorophores. The SOFI image thus is comparable to an image recorded with a microscope with a PSF of $U^{2}\left(\mathbf{r}-\mathbf{r}^{\prime}\right)$, the square of the original PSF $U\left(\mathbf{r}-\mathbf{r}^{\prime}\right)$ with an additional prefactor. In general, the $n^{\text {th }}$ order SOFI image is proportional to the $n^{\text {th }}$ power of the PSF:

$$
S_{n}(\mathbf{r}) \propto \sum_{j=1}^{N} U^{n}\left(\mathbf{r}-\mathbf{r}_{j}^{\prime}\right) \epsilon_{j}^{n} .
$$

The resolution enhancement in all dimensions results from this shrinking of the PSF. Without additional processing steps and assuming a Gauss-shaped PSF, $n^{\text {th }}$ order SOFI consequently increases the resolution by a factor of $\sqrt{n}$. The actual achievable resolution depends on measurement time and blinking behavior. The more transitions from the on- to the off-state and back a movie contains for each individual fluorophore, the higher is the order of the SOFI image that can be reliably calculated from it.

Unless specified otherwise, all SOFI images in this work are calculated to second order.

\subsection{Computation and correction for bleaching}

The SOFI algorithm used for the calculations in this work is based on the principles described above. A few alterations were implemented, for example to correct for bleaching. The simplest algorithm for calculating an $n^{\text {th }}$ order SOFI image from a 
movie $F(\mathbf{r}, t), t=1 \ldots N$ of a fluorescently labeled sample with $N$ frames is given by

$$
S_{n}\left(\mathbf{r} ; \tau_{1}, \ldots, \tau_{n-1}\right)=C_{n}\left[F(\mathbf{r}, t), F\left(\mathbf{r}, t+\tau_{1}\right), \ldots, F\left(\mathbf{r}, t+\tau_{1}+\cdots+\tau_{n-1}\right)\right] .
$$

The time lags $\tau_{1}, \ldots, \tau_{n-1}$ are fixed user selected values, replacing the time-consuming calculation of the integral with the evaluation of the $n^{\text {th }}$ order cumulant at only one point. To remove shot noise, at least one time lag must be chosen $>0$. On the other hand, the correlation, and with it the signal strength, decreases with increasing $\tau_{1}$. $\tau_{1}=\tau_{2}=\cdots=\tau_{n_{1}}=1$ has proven to be a good choice for most cases, providing shot noise removal, good signal, and low computational effort.

Photobleaching of fluorophores is a problem for most fluorescence imaging techniques and SOFI is no exception. Bleaching causes emitters to be transferred irretrievably to a dark state, thereby lowering the average intensity of the individual images in a movie with time. However, the stationarity of the blinking emitters is a prerequisite for Eq. 8 to be valid. A movie of constantly emitting fluorophores does not yield a SOFI image, causing non-fluctuating fluorescence to vanish. In contrast, an image, albeit without the desired resolution enhancement, can be obtained by applying the SOFI algorithm to a movie of bleaching emitters. Considering raw data with both blinking and bleaching, the bleaching result reduces the resolution of the true SOFI image calculated from the blinking. To eliminate this problem, the movie is sectioned into portions from which the cumulants are calculated. These windows are small compared to the time scale of bleaching, but large compared to the time scale of blinking. As a result, the average intensity can be considered constant. The SOFI image of a movie sectioned into $W=\left\lfloor N / N_{w}\right\rfloor$ windows of $N_{w}$ frames is the average of all $W$ individually calculated SOFI images:

$$
\begin{aligned}
& S_{n}\left(\mathbf{r} ; \tau_{1}, \ldots, \tau_{n-1}, N_{w}\right) \\
& \quad=\frac{1}{W} \sum_{w=1}^{W} C_{n}^{w}\left[F(\mathbf{r}, t), F\left(\mathbf{r}, t+\tau_{1}\right), \ldots, F\left(\mathbf{r}, t+\tau_{1}+\cdots+\tau_{n-1}\right)\right] .
\end{aligned}
$$

If the blinking is slow with regard to the frame rate or distributed over different time scales, a hierarchical algorithm can be used. Here, a cumulant image for a fixed set of time lags is calculated. Then, by addition of adjacent frame pairs, a movie with half the frame number as the original image is generated. From this new movie, the cumulant is calculated once more, and adjacent frame pairs are added up again. This procedure is repeated until all information has been extracted, and all resulting SOFI images are summed up into the final picture. 


\subsection{Effective pixel size}

The effective pixel size, the area of the sample recorded by one camera pixel, is an important factor for SOFI quality. Distributing fluorescence light of one emitter over a large number of pixels typically decreases the signal-to-noise ratio and thus the image quality. Depending on the brightness of the fluorophores, around $80 \mathrm{~nm}$ typically is the minimum effective pixel size recommendable for SOFI imaging. Since this limits the achievable resolution of SOFI, a way to increase the pixel number and to decrease the effective pixel size is highly desirable. One approach to solve this is crosscorrelation SOFI (XC-SOFI) 17. Here, each pixel is not only autocorrelated, but crosscorrelated with neighboring pixels. Thus, additional pixels (XC-pixels) carrying true information are generated in between the original pixel and its neighbors (AC-pixels) to make full use of the increased resolution.

There are, however, several drawbacks. Apart from the generally very large computational effort, there is more than one way to correlate the original pixels to generate the same number of XC-pixels in the same positions. Although XC-pixels in the same positions but calculated by correlating different original pixels show slight differences in their intensities, the theory provides no explanation as to which way is optimal. Furthermore, the intensity of the crosscorrelation pixels is weighted with a factor that depends on the distance of the two correlated pixels and the PSF. This distance factor has to be counterbalanced, otherwise the intensity of the $\mathrm{XC}$-pixels is on a different scale than the intensity of the AC-pixels, leading to grid artifacts. Using an estimation of the distance factor, it can be compensated, but this is not straightforward and typically, artifacts remain even after correction.

An exact solution to the problem is to increase the pixel size of the recorded images $F(\mathbf{r}, t)$ in Fourier space before SOFI calculation, using the fact that the optical transfer function (OTF), the Fourier transform of the PSF, has a finite support. The OTF therefore is zero for sufficiently large values of the Fourier vector. The same holds true for the Fourier transformed images $\tilde{F}(\mathbf{k}, t)$, if the original effective pixel size is smaller than the diffraction limit. As a consequence, it is possible to Fourier transform a recorded image, to increase the size of the Fourier transform by adding zeros to the borders, and to transform it back. The result is an image with the same image content as the original image, but increased pixel number and reduced pixel size. Carrying out this Fourier supersampling on a whole movie prior to SOFI analysis leads to a SOFI image with increased pixel number, able to display the additional resolution gained by using SOFI. 
Autocorrelation SOFI with bleaching correction and Fourier supersampling is referred to as basic SOFI throughout this work.

\section{$2.4 \quad$ Fourier reweighting}

As detailed earlier, the SOFI PSF is proportional to the $n^{\text {th }}$ power of the original PSF, leading to a resolution enhancement of factor $\sqrt{n}$ when approximating the PSF by a Gaussian function (see Eq. 8). Correspondingly, the support of the SOFI OTF $\tilde{U}(\mathbf{k})$, while still finite, is widened by factor $n$. The PSF of second order SOFI therefore corresponds to the original PSF multiplied with itself. In Fourier space, this means the SOFI OTF is a convolution of the OTF with itself, with consequently doubled support. However, the OTF shape differs from the shape of the original OTF, for example with very low amplitudes of the frequencies at the rims. To make full use of the doubled support, the amplitudes have to be adjusted to reshape the OTF. The PSF resulting from this intensity reweighting in Fourier space is narrower than the SOFI PSF and of the shape of the original PSF. This is illustrated in Fig. 5 .

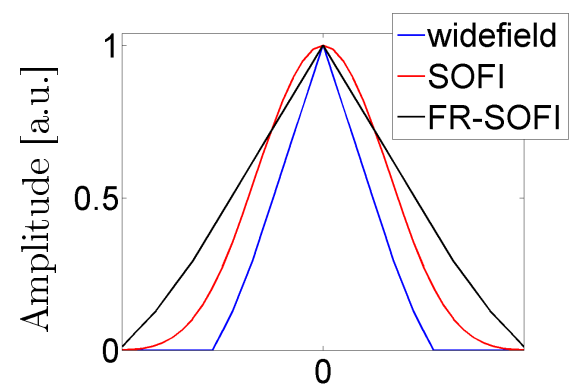

Spatial frequency

(a) Fourier space: optical transfer function $(\mathrm{OTF})$

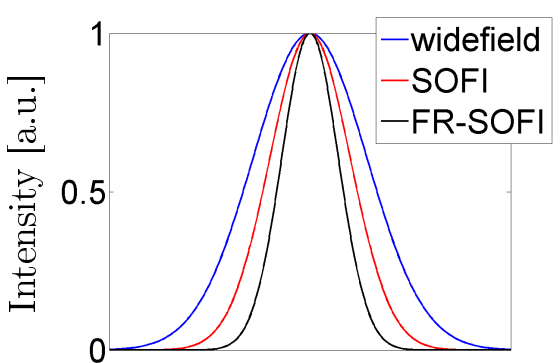

Position

(b) Image space: point spread function (PSF)

Figure 5. Widefield, SOFI and Fourier-reweighted SOFI OTF (a) and PSF (b). Second order SOFI doubles the OTF support, corresponding to a doubling of spatial information. In image space, the doubled resolution can be displayed after Fourier-reweighting of the spatial frequencies. This corresponds to a reduction of the FWHM of the PSF by a factor of $\sqrt{2}$ for second order SOFI and a factor of 2 for Fourier-reweighted second order SOFI.

Compressing the PSF to $U(n \cdot \mathbf{r})$ leads to a stretched OTF

$$
\mathcal{F}(U(n \cdot \mathbf{r}))=\tilde{U}\left(\frac{\mathbf{k}}{n}\right)
$$


while the support remains unchanged. The visible resolution of an image with the PSF $U(n \cdot \mathbf{r})$ is increased by factor $n$ compared to an image with the PSF $U(\mathbf{r})$. In the same way, the SOFI OTF can be reweighted with a function that is based on the original OTF, according to

$$
\begin{aligned}
& S_{n}(\mathbf{r}) \stackrel{\mathcal{F}}{\longrightarrow} \tilde{S}_{n}(\mathbf{k}) \\
& \qquad \cdot \sqrt{\tilde{U}\left(\frac{\mathbf{k}}{n}\right)} \frac{\underbrace{\tilde{U}(\mathbf{k}) * \tilde{U}(\mathbf{k}) * \cdots * \tilde{U}(\mathbf{k})}_{n \text {-times }}+\alpha}{}, \\
& S_{n}^{\prime}(\mathbf{r}) \stackrel{\mathcal{F}^{-1}}{\longleftarrow} \tilde{S}_{n}^{\prime}(\mathbf{k})
\end{aligned}
$$

were $\alpha>0, \alpha \in \mathbb{R}$ is a small positive constant to prevent division by zero. As a result, the resolution enhancement is increased to the factor $n$. By performing this Fourier reweighting on the SOFI images, the SOFI PSF is changed in Fourier space to be of the same functional form of the original PSF 17.

\subsection{Linearization of the intensity response}

According to Eq. 8, emitters contribute with the $n^{\text {th }}$ power of their brightness $\epsilon_{j}$ to the SOFI image, potentially suppressing dark areas relative to brighter ones. The brightness response of the SOFI image is linearized by minimizing the least squares cost function

$$
\operatorname{LSQ}\{g(\mathbf{r})\}=\sum_{\mathbf{r}}\left(U^{n}(\mathbf{r}) * g^{n}(\mathbf{r})-S_{n}(\mathbf{r})\right)^{2}
$$

with the SOFI order $n$, the PSF $U(\mathbf{r})$, the distribution of emitters on the sample $g(\mathbf{r})$, and the SOFI image $S_{n}(\mathbf{r})$. To prevent the recovery of unphysical frequencies, the constraint is placed that the support of $g$ in Fourier space does not exceed that of the SOFI PSF $U^{n}$. The problem is solved using few iterations of a nonlinear conjugate gradient minimizer. Positivity and finite support of the solution are enforced after every iteration. To improve the visual quality of the result, the level of the residual background in the SOFI image is determined by visual inspection. Pixels below the threshold are set to zero before the optimization.

fSOFI is photobleaching corrected AC-SOFI (basic SOFI) with additional Fourier supersampling, Fourier reweighting and intensity linearization. All SOFI images in this work are fSOFI images, if not specified otherwise. 


\subsection{Fluorescence intermittency requirements}

SOFI has been shown to be very robust regarding the fluorescence intermittency of the labels 21 . A key quantity when comparing different types of blinking is the rate ratio $r$. It denotes the ratio of the duration of the average on- and off-states of a label and is defined as

$$
r=\frac{k_{o f f}}{k_{o n}}=\frac{\tau_{o n}}{\tau_{o f f}}
$$

with the rate for off-switching $k_{o f f}=\tau_{o f f}^{-1}$ and the rate for on-switching $k_{o n}=\tau_{o n}{ }^{-1}$, with $\tau_{\text {off }}$ and $\tau_{\text {on }}$ the half lifes of the on- and off-states, respectively. Simulations 21] showed that the rate ratio can be chosen almost arbitrarily for second order SOFI, although a higher number of switching events per time reduces the total imaging time per SOFI image. For higher orders, the quality of the images and thus the achievable resolution enhancement depends on the combination of SOFI order and rate ratio. For example, for odd higher orders the blinking behavior must be asymmetric $(r \neq 1)$. In summary, any rate ratio can be used for second order SOFI, while for higher orders, the order that best fits the blinking behavior can be chosen.

The optimal exposure time depends mainly on $\tau_{\text {off }}$. Ideally, the on-time of an emitter lasts about two to five frames, leading to a frame rate between 2 and 5 $k_{o f f}$. Provided a sufficient signal-to-noise ratio, on-times longer than that typically yield good results as well. In general, fast blinking is favorable, as it ensures a large number of blinking events in a short time and thus reduces overall imaging time. However, even if the blinking is far from the ideal, this can usually be compensated by recording more frames per movie. Due to this robustness, the labels for SOFI can be chosen according to the requirements of the application from a large variety with very different blinking mechanisms.

QDot blinking covers many different time scales with little possibility to influence the behavior. Nevertheless, the flexibility of SOFI and the fact that QDots are very bright, stable labels make it a very useful label for SOFI. In contrast, the photoswitching of organic dyes can be tuned to generate on- and off-times according to SOFI requirements. Both QDots and organic dyes are typically used to immunostain fixed cells. Reversibly switchable fluorescent proteins (RSFPs) are proteins that change from a fluorescent to a dark state and back upon irradiation with light of a suitable wavelength, potentially allowing for the induction of tunable fluctuations and opening up the possibility of a live-cell compatible label for SOFI. 


\subsection{Setup requirements}

SOFI's increased lateral resolution and background suppression is achieved by recording a movie of blinking emitters on a standard widefield microscope and subsequent SOFI calculation. A microscope for SOFI data acquisition should be equipped with a camera that is sensitive and fast enough to record the blinking dynamics of the labels. In general, this means it should be capable of frame rates of $20-100 \mathrm{~Hz}$. Regarding the magnification, a resulting effective pixel size of 80 - $100 \mathrm{~nm}$ is ideal. For pixels smaller than that, the signal-to-noise-ratio (SNR) is negatively affected, since the photons emitted by a single fluorophore are distributed over too many pixels. Bigger effective pixels, on the other hand, lead to a resolution of individual PSFs on the camera chip that is insufficient for reliable PSF estimation for Fourier reweighting and linearization. In both cases, the achievable resolution is suboptimal. Regarding light sources, the choice between lasers and LED engines depends on the type of fluorophore. 


\section{SOFI with Quantum Dots}

\subsection{Quantum Dots}

Quantum Dots (QDots) are semiconductor crystals with diameters between 5 and $50 \mathrm{~nm}$ that can be used as bright and stable labels in immunostaining protocols. Due to their size, they have unique properties that can be described as being in between those of bulk and molecular forms.

Their fluorescence stems from the relaxation of an excited electron to the ground state, where it recombines with an electron hole. Since the band gap between the ground state and the excited state depends on the size of the QDot, so does the wavelength of the subsequently emitted light. The typical distance between an electron and the electron hole it is bound to is called the bulk exciton Bohr radius. The extent of bulk material is much larger than this distance. As a result, the energy levels are continuous, forming a filled valence band and an empty conduction band, separated by a band gap. In this case, electrons can be excited from their ground state in the valence band to their excited state in the conduction band, leaving an electron hole in the valence band. The radii of QDots are typically in the range of, or smaller than, the bulk exciton Bohr radius $22 \mid 23$, which leads to quantum confinement. The energy levels in this confined case are discrete and the band gap is larger than in bulk material. The more closely confined the exciton is, the larger is the band gap. Therefore, the smaller the particle, the more blue shifted is the fluorescence. By generating QDots with different sizes, the whole visible spectrum can be covered. As excitation bands are typically in the range of 350 to $450 \mathrm{~nm}$ with only a slight dependence on the band gap, the emission wavelength instead of the excitation wavelength is given in the designation of a QDot.

QDots show inherent blinking. This blinking is a problem for many applications, but can be used to great effect with SOFI 16/1724 26. There are several theories to explain the exact nature of the processes involved in QDot blinking. Roughly, it is caused by exciton generation not followed by recombination and fluorescence, but by ejection of a charge carrier from the core. Non-radiative recombination of subsequently generated excitons is then induced by the charge remaining in the core, rendering the QDot dark and non-fluorescent. After a certain time, the ejected carrier returns to the core, where it recombines with the remaining carrier. Without the charge in the core, excitons recombine emitting light once more, and thus fluorescence is restored ${ }^{27}$. The probability distribution of both on- and off- peri- 
ods is described by a power law dependence, suggesting distributed kinetics $28[29$. Consequently, QDot blinking covers a large range of on- and off-times.

\subsection{Optimization of the imaging parameters}

To achieve a high image quality for the biological applications, it was necessary to optimize the imaging parameters as well as the setup, a widefield microscope with LEDs as light source. As a starting point I chose the conditions previously published for second order SOFI imaging with QDots ${ }^{16}$, which were typically 5000 frames at $100 \mathrm{~ms}$ frame time. Under these conditions, data acquisition for one SOFI image took approximately $8 \mathrm{~min} 20 \mathrm{~s}$. Since further analysis required data sets consisting of images of a minimum of five z-positions in two color channels, recorded successively, the imaging time per neuron amounted to a total of one and a half hours, which is inconvenient by itself. Moreover, most setups are not sufficiently stable over such a period of time, resulting in sample drift during measurements.

Lateral and axial sample drift severely reduce the resolution achieved by SOFI, and decrease the accuracy of further analysis dramatically. I therefore tested whether the frame number and exposure time could be reduced to minimize overall imaging time, and introduced setup modifications to increase the mechanical stability of the setup, minimizing drift during data acquisition.

\subsubsection{Exposure time optimization}

Due to the much larger range of on- and off-times covered by QDots than by organic dyes and fluorescent proteins, there can be no ideal fit between exposure time and blinking kinetics. The exposure time is thus best chosen to fit other criteria. Although short exposure times reduce the total imaging time, exposure times need to be long enough to ensure an adequate signal-to-noise ratio (SNR) for each individual movie frame. Exemplary studies on $\mathrm{QD}_{525^{-}}$and $\mathrm{QD}_{605^{-}}$labeled rat hippocampal neurons (Fig. 6) showed no disadvantage in SNR or quality of the SOFI image of an exposure time of only $50 \mathrm{~ms}$ compared to the previously published $100 \mathrm{~ms}$.

\subsubsection{Frame number optimization}

The quality of a SOFI image depends strongly on the number of frames used for the calculation. Among the parameters influencing the ideal frame number are the 

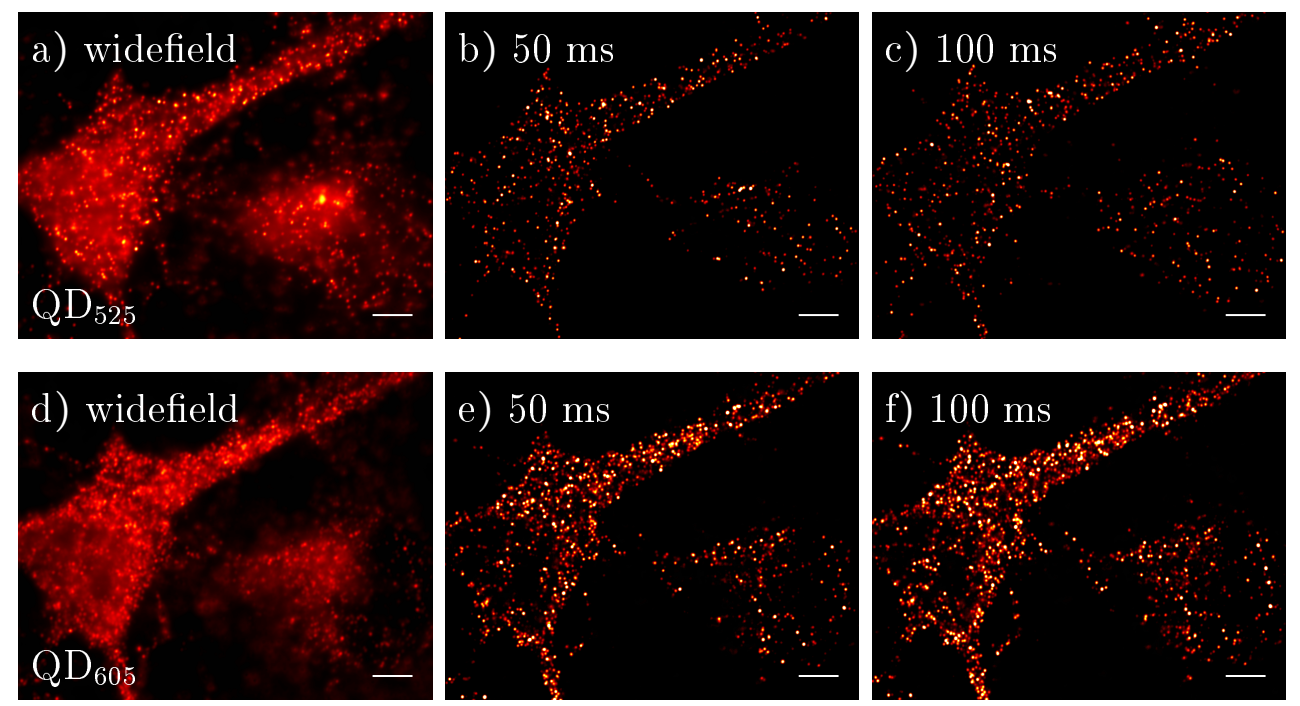

Figure 6. Widefield image (a, d), and basic SOFI image calculated from movies with 50 $\mathrm{ms}(\mathrm{b}, \mathrm{e})$ and $100 \mathrm{~ms}(c, f)$ exposure time, with $Q D_{525}$ (top) and $Q D_{605}$ (bottom) as labels.

The image quality for 50 and $100 \mathrm{~ms}$ frame time is identical. Scale bar: $4 \mu \mathrm{m}$.

kinetics of the fluorescence intermittency, the brightness of individual fluorophores, and the order of SOFI.

A fluorophore needs to change its fluorescence state a certain number of times over the course of the data recording to become visible in the SOFI image. QDots, with their highly variable blinking, show a great variety in the number of switching events per time period. Consequently, there is the same variety in the number of frames that is required for the detection of individual QDots. To find a frame number which allows for the detection of most of the QDots in a sample, I made use of the visibility of individual QDots in SOFI images of sparsely stained samples. Examination of the QDot visibility in SOFI images calculated from 1000 to 4000 frames (Fig. 7) revealed that 3000 frames are well sufficient for second order SOFI.

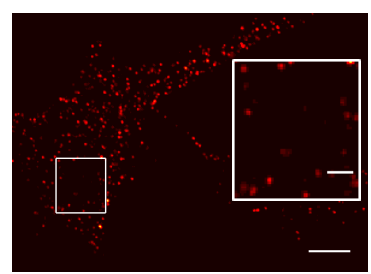

(a) 1000 frames

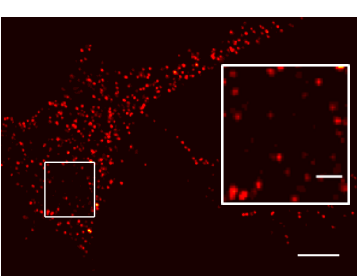

(b) 2000 frames

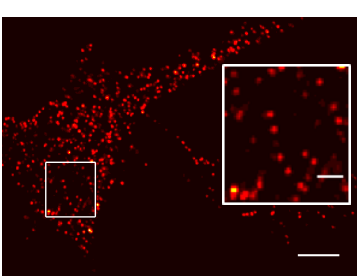

(c) 3000 frames

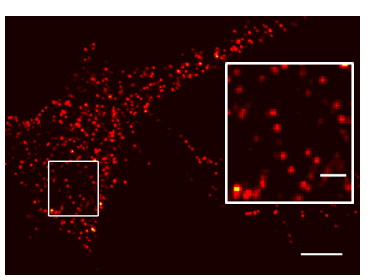

(d) 4000 frames

Figure 7. Basic SOFI images of $Q D_{525}$-stained neurons, calculated from different numbers of frames. 3000 frames are necessary to detect all emitters. Scale bar: $4 \mu \mathrm{m}$, inset $1 \mu \mathrm{m}$. 
Using more frames does not lead to a further increase of visible QDots or the overall image quality.

\subsubsection{Drift reduction}

Sample drift is a challenge common to many superresolution techniques, where highest accuracy is the goal. Therefore, highest sample stability over long periods of time is a necessity.

\subsubsection{Axial drift reduction}

When the distance between the objective lens and the sample gradually changes, the sample drifts out of focus. Typical reasons for this axial drift are the settling of the sample, the sample stage or the objective lens to an equilibrium position, or external disturbances. Ideally, the objective lens settles jointly with the focusing mechanism (the z-drive) of the microscope to an equilibrium position, but there is the possibility of slow, persistent movement of the focusing mechanism caused by depression by the weight of the objective lens, leading to constant defocussing. Since typical imaging times for many applications of widefield imaging are on time scales much shorter than the defocussing, this effect often poses no difficulty.

In contrast, the acquisition of 3D, dual-color SOFI data sets is negatively affected by axial drift, as it involves the more time consuming recording of z-stacks of movies in two color channels. Axial drift during the recording of a movie corrupts the resolution of the corresponding SOFI image. Furthermore, since the color-channels are not recorded simultaneously, z-drift not only causes deviation of the z-positions

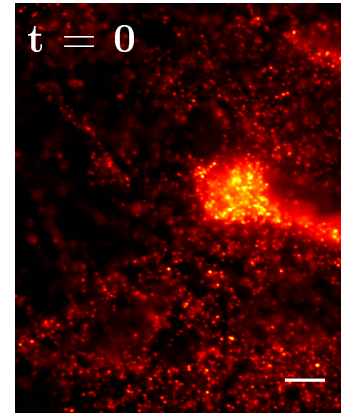

(a) Defocusing by axial drift over 30 min
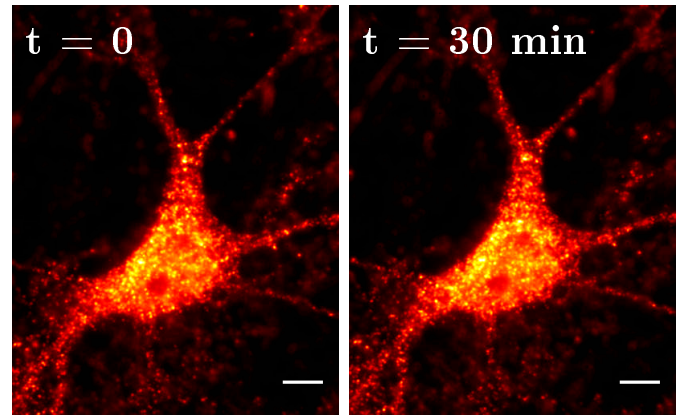

(b) Setup modifications prevent axial drift

Figure 8. Axial drift over $30 \mathrm{~min}$, before (a) and after (b) setup modifications. The strong defocussing by axial drift was eliminated. Scale bar: $5 \mu \mathrm{m}$. 
of the two color channels, but also a shift of the individual images of the z-stacks from their nominal z-positions. Obviously, such data is of little use.

The settling of objective lens, sample and microscope components probably caused the strong initial drift in the first minutes after placing the sample. However, the drift, measured by focusing using the z-drive and refocusing after a certain period of time using the piezo-z-scanner, was continuous, with the z-position changing up to $3 \mu \mathrm{m}$ per hour. This implied a constant sinking of the objective lens, which was ultimately prevented by mechanically fixing the z-drive in position. The initial drift can be avoided by simply allowing the setup and sample to settle for several minutes before starting the data acquisition. Figure 8 a gives an example of strong defocussing of a $\mathrm{QD}_{605}$-labeled neuron over the course of $30 \mathrm{~min}$. Many of the unspecifically bound QDots that are in focus at the beginning of the measurement are out of focus after $30 \mathrm{~min}$, and vice versa. In contrast, the images recorded after the setup modifications (Fig. 8b) are completely stable.

\subsubsection{Lateral drift reduction}

(a) Initial drift

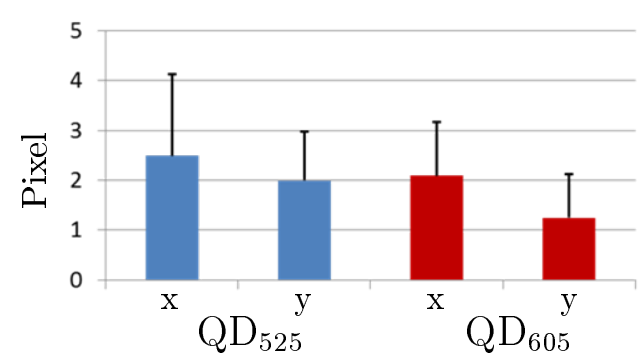

(b) Drift after stabilization

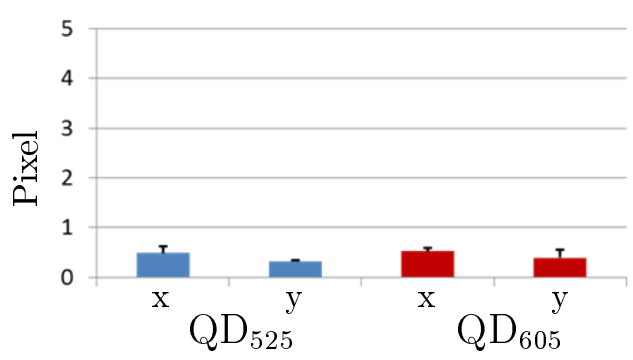

Figure 9. Lateral drift reduction due to shorter imaging time and setup modifications. Initial drift of SOFI raw data (a), and considerably reduced drift after shortening of the imaging time and stabilization of the setup (b). Effective pixel size: $100 \mathrm{~nm}$.

Excluding external disturbances like vibrations caused by nearby equipment, lateral sample drift is typically caused by instabilities of the sample stage. Like axial drift, lateral drift is highly detrimental to the quality of SOFI images. Additionally, it renders the correlation of two color channels to determine the relative location of the labeled proteins impossible. Consequently, the original lateral sample drift of up to $250 \mathrm{~nm}$, or 2.5 pixels (Fig. 9a, calculated from 4 data sets á 7 z-positions), had to be reduced. This was achieved by reducing the exposure time from $100 \mathrm{~ms}$ 
to $50 \mathrm{~ms}$ (3.2.1) and the frame number from 5000 to 3000 (3.2.2), thereby reducing the overall imaging time, and installation of a custom-built sample stage designed for high stability. The lateral sample drift was thus reduced to a tolerable maximum $50 \mathrm{~nm}$, or 0.5 pixels in x-direction and less in y-direction (Fig. 9p, calculated from 2 data sets à 7 z-positions).

\subsubsection{Discussion}

Due to the great variety of QDots regarding on- and off-times, the exposure time can be chosen more flexibly than with other types of labels, and exposure times of 50 and $100 \mathrm{~ms}$ proved equally well suited for SOFI QDot imaging. Similarly, the number of switching events occurring to a QDot per time period covers a broad range. Since a certain number of switching events is necessary for an emitter to become visible in the SOFI image, a certain minimum of frames has to be analyzed to detect even the QDots that were switching extremely infrequently during movie acquisition. For this, 3000 frames were determined to be entirely sufficient. As a result, the imaging time could be reduced to one third of the original time.

Combined with the improved setup stability provided by restricting the mobility of the z-drive and installing a custom-made sample stage, the high image quality and sample stability required for biological applications of 3D, dual-color SOFI could thus be ensured.
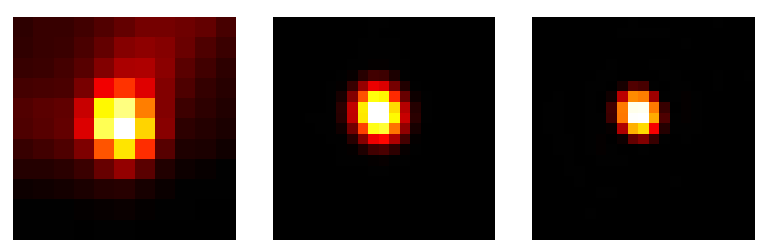

(a) Widefield

(b) basic SOFI

(c) FR-SOFI

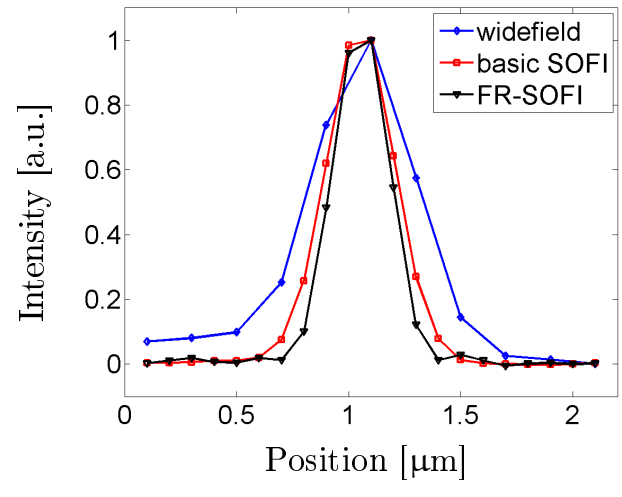

(d) Profile in x-direction

Figure 10. Widefield (a), basic SOFI (b), FR-SOFI image (c) of a single Quantum Dot with emission wavelength $\lambda_{e m}=525 \mathrm{~nm}$, and profiles in $\mathrm{x}$-direction (d). The effective pixel size is $100 \mathrm{~nm}$ for the widefield image and $50 \mathrm{~nm}$ for the SOFI images. 


\subsection{Resolution improvement}

Second order SOFI is capable of reducing the size of the PSF by a factor of $\sqrt{2}$ compared to widefield imaging, thereby increasing the resolution by the same factor. With additional Fourier reweighting, the total resolution can even be doubled. Subsequent linearization of the intensity response is designed to compensate for the increase of emitter brightness differences introduced by SOFI.

Figure 10 exemplifies the resolution improvement from conventional widefield imaging to basic SOFI and to Fourier reweighted SOFI (FR-SOFI) with images of the PSF of a single $\mathrm{QD}_{525}$ and the corresponding profiles in x-direction. The average FWHM of the Gaussian fits of the PSFs of Quantum Dots with emission wavelength $\lambda_{e m}=525 \mathrm{~nm}$ and $605 \mathrm{~nm}$ for different processing types are given in Table 1 .

\begin{tabular}{|c|c|c|c|c|c|c|}
\hline & widefield & & basic SOFI & & FR-SOFI & improvement \\
\hline $\mathrm{QD}_{525}$ & $264 \pm 14 \mathrm{~nm}$ & $\stackrel{1.42}{\longrightarrow}$ & $186 \pm 8 \mathrm{~nm}$ & $\stackrel{1.27}{\longrightarrow}$ & $146 \pm 3 \mathrm{~nm}$ & 1.81 \\
\hline $\mathrm{QD}_{605}$ & $296 \pm 20 \mathrm{~nm}$ & $\stackrel{1.38}{\longrightarrow}$ & $214 \pm 17 \mathrm{~nm}$ & $\stackrel{1.32}{\longrightarrow}$ & $162 \pm 6 \mathrm{~nm}$ & 1.82 \\
\hline
\end{tabular}

Table 1. Comparison of the Gauss fitted PSF FWHM of single QDots for different stages of SOFI processing, calculated from ten emitters. For both emission wavelengths, SOFI increases the resolution by the expected factor of $\sqrt{2}$. Total resolution improvement of SOFI with additional Fourier reweighting amounts to a factor of $\sim 1.8$.

The resolution enhancement from widefield imaging to basic SOFI is in excellent agreement with the expected factor of $\sqrt{2}$. Fourier reweighting provides a further increase of factor $\sim 1.3$, yielding a total improvement factor of $\sim 1.8$. While the theoretically achievable improvement from basic SOFI to FR-SOFI is an additional factor of $\sqrt{2}$, the complete information can only be extracted from hypothetical noise-free data, limiting the resolution improvement slightly.

Using SOFI, structures can be resolved that are blurred in the corresponding widefield image (Fig. 11). Additionally, SOFI strongly reduces the background, which is typically high in widefield images and hinders the extraction of information. In several areas, basic SOFI thus revealed separate, single QDots which were not visible in the widefield image. Fourier reweighting and linearization improved the image further. This is made especially clear by the profile of the two QDots (Fig. 11e, which, in contrast to conventional imaging, are well resolved by SOFI. 


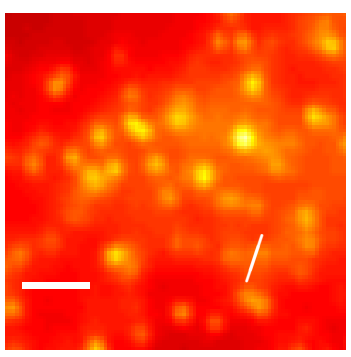

(a) widefield

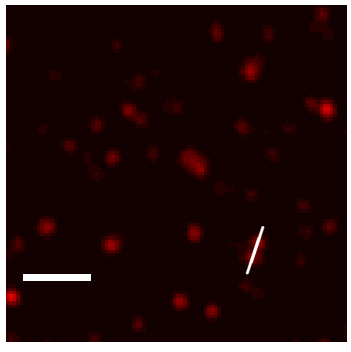

(b) basic SOFI

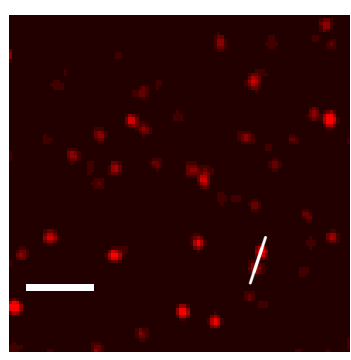

(c) FR-SOFI

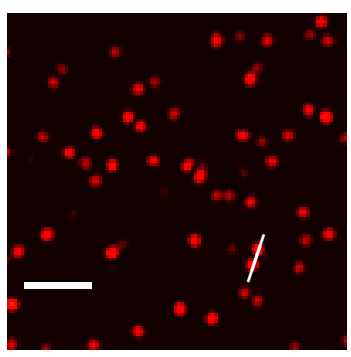

(d) lin. FR-SOFI

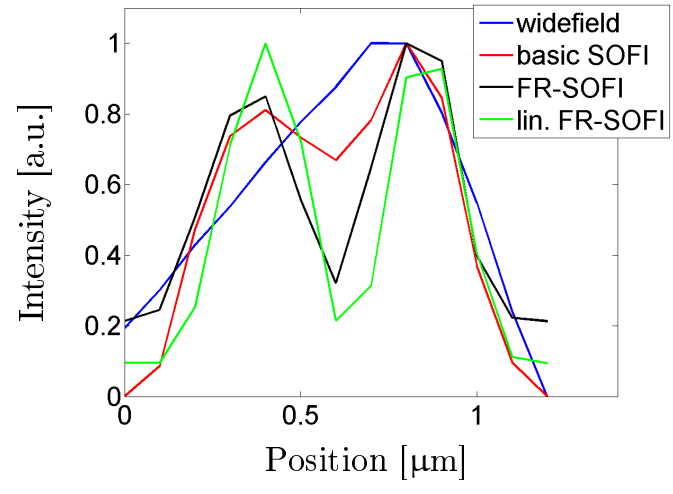

(e) Intensity profile

Figure 11. Widefield (a), basic SOFI (b), FR-SOFI (c), linearized FR-SOFI image (d) of a sample of $Q D_{525}$. Scale bar: $1 \mu \mathrm{m}$. Intensity profile (e) of two QDots in the lower left corner resolved by SOFI but not by widefield microscopy.

\subsubsection{Discussion}

The measured resolution improvement provided by SOFI is in very good agreement with the theoretical predictions. However, the PSFs of conventional imaging for both types of emitters are larger than calculated. According to Eq. 2, the FWHM of the PSF of $\mathrm{QD}_{525}$ and $\mathrm{QD}_{605}$ is, for ideal conditions, $191 \mathrm{~nm}$ and $220 \mathrm{~nm}$, respectively. Although deviations from the ideal values due to imperfections in the optics and a slight refractive index mismatch are expected, an original PSF closer to the diffraction limit would improve the widefield and consequently the SOFI resolution, allowing for the elucidation of structures and processes on a smaller scale. 


\section{$3.4 \quad \mathrm{GABA}_{\mathrm{B}}$ receptor subunit trafficking}

\subsubsection{The $\mathrm{GABA}_{\mathrm{B}}$ receptor}

Memory acquisition and information storage in the brain is thought to be founded on the modification of the efficiency of neuronal signal transduction, which is called synaptic plasticity 20. The synaptic efficacy can be modulated for example by changes of the affinity of neurotransmitter receptors to their ligands, by alterations of the kinetics of further signaling, like the opening and closing of ion channels, or by variations in the availability of the neurotransmitter receptors.

Most synaptic transmissions in the nervous system of vertebrates are mediated by glutamate receptors and $\gamma$-aminobutyric acid receptors (GABARs). $\gamma$-Aminobutyric acid (GABA), one of the most important inhibitory neurotransmitters in the central nervous system, typically reduces the probability of action potential generation and thus prevents excess stimulation. Of the two types of GABARs, the ionotropic $\mathrm{GABA}_{\mathrm{A}}$ receptor is a ligand-gated chlorine channel, while the metabotropic $\mathrm{GABA}_{\mathrm{B}}$ receptor mediates further signal transduction by G-protein activation upon ligandbinding. The results of this further signaling, such as the activation of ligand-gated potassium channels and the decrease in the opening probability of ligand-gated calcium channels, are inhibitory in nature. Therefore, the availability of the GABA $_{B}$ receptor in the dendritic plasma membrane is an important factor in action potential generation as well as synaptic plasticity, and the details of its trafficking are of great interest.

The $\mathrm{GABA}_{\mathrm{B}}$ receptor is a heteromer consisting of the subunits $\mathrm{GABA}_{\mathrm{B}} \mathrm{R} 1$ and $\mathrm{GABA}_{\mathrm{B}} \mathrm{R} 2$ [30. Both are expressed separately in the soma and initially reside in the somatic endoplasmic reticulum (ER) membrane. From there, they are transported to their sites of insertion into the plasma membrane of a dendrite. GABA $_{B} R 1$ features the GABA binding site and an ER retention motif, which prevents the subunit from leaving the ER membrane. GABA $\mathrm{R} 2$ is coupled to the G-protein and is able to mask the ER retention motif of $\mathrm{R} 1$ with its $\mathrm{C}$-terminus. As a result, $\mathrm{R} 1$ is able to leave the ER only when coupled to R2. This ensures that only GABA binding sites that are properly coupled to the G-protein, and thus are able to sustain the signal, are integrated into the plasma membrane of the dendrite. The details of this transport are an important addition to our understanding of the nervous system.

There are two alternatives for the transport of the $\mathrm{GABA}_{\mathrm{B}}$ receptor subunits. One possibility is assembly close to the soma and transport along the dendrite in a 


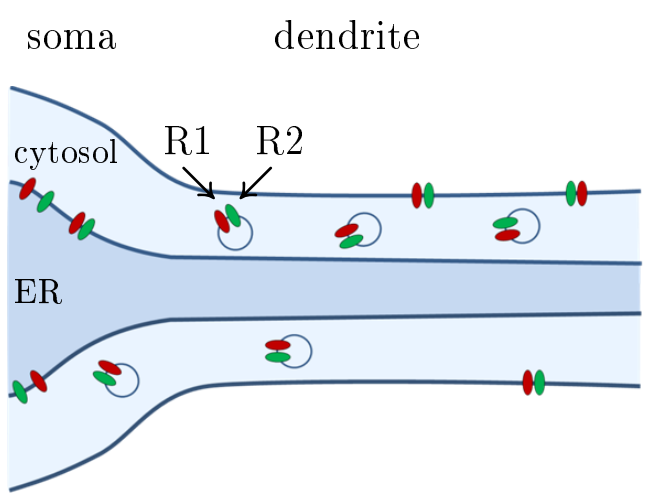

(a) Early assembly and joint trafficking

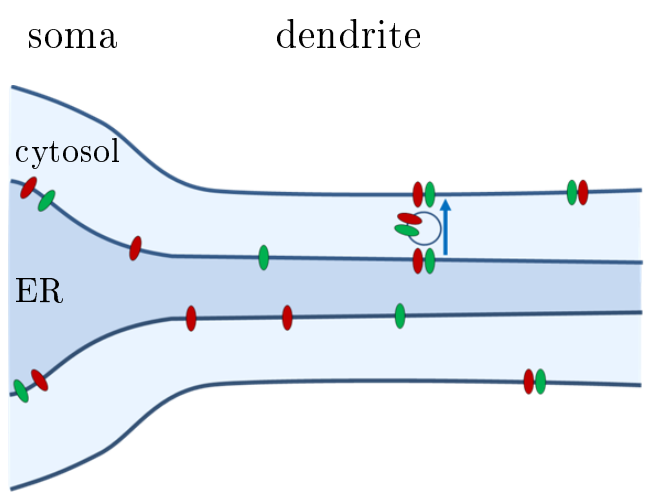

(b) Late assembly and separate trafficking

Figure 12. Schematic of a part of the soma and a dendrite of a neuron, illustrating the alternative ways of $G A B A_{B}$ receptor subunit trafficking. $R 1$ and $R 2$ either assemble close to the soma, leave the ER membrane and are transported in vesicles along the dendrite in the cytosol (a), or the subunits are transported separately along the ER membrane (b).

By studying the colocalization of the subunits, both alternatives can be distinguished.

vesicle as a complete receptor (Fig. 12a). The second possibility is separate transport of both subunits along the ER membrane. In this scenario, both subunits only leave the ER membrane in favor of a vesicle already close to their site of insertion to the plasma membrane, and the subunits cover only a short distance as an assembled receptor (Fig. 12b). These two ways can be distinguished by colocalization analysis. The colocalization of $\mathrm{GABA}_{\mathrm{B}} \mathrm{R} 1$ and $\mathrm{R} 2$ in either case is maximal at the plasma membrane, but the extent of subunit colocalization in the cytoplasmic region of the dendrite differs. For the case of an early assembly and joint vesicular travel through the cytosol, the colocalization in the volume is approximately equal to that in the plasma membrane. For separate travel, the colocalization is lower in the volume of the dendrite than in the plasma membrane.

Studies using colocalization analysis on confocal fluorescence images 31 and other biochemical techniques found differential mobility of the subunits, indicating separate trafficking and late assembly to the complete neurotransmitter receptor. Additional analysis using SOFI images benefits from the increase in lateral and axial resolution and the background suppression, which simplifies identifying the relevant signals and promises greater colocalization accuracy. 


\subsubsection{D, dual-color SOFI of $\mathrm{GABA}_{\mathrm{B}} \mathrm{R} 1$ and $\mathrm{R} 2$}
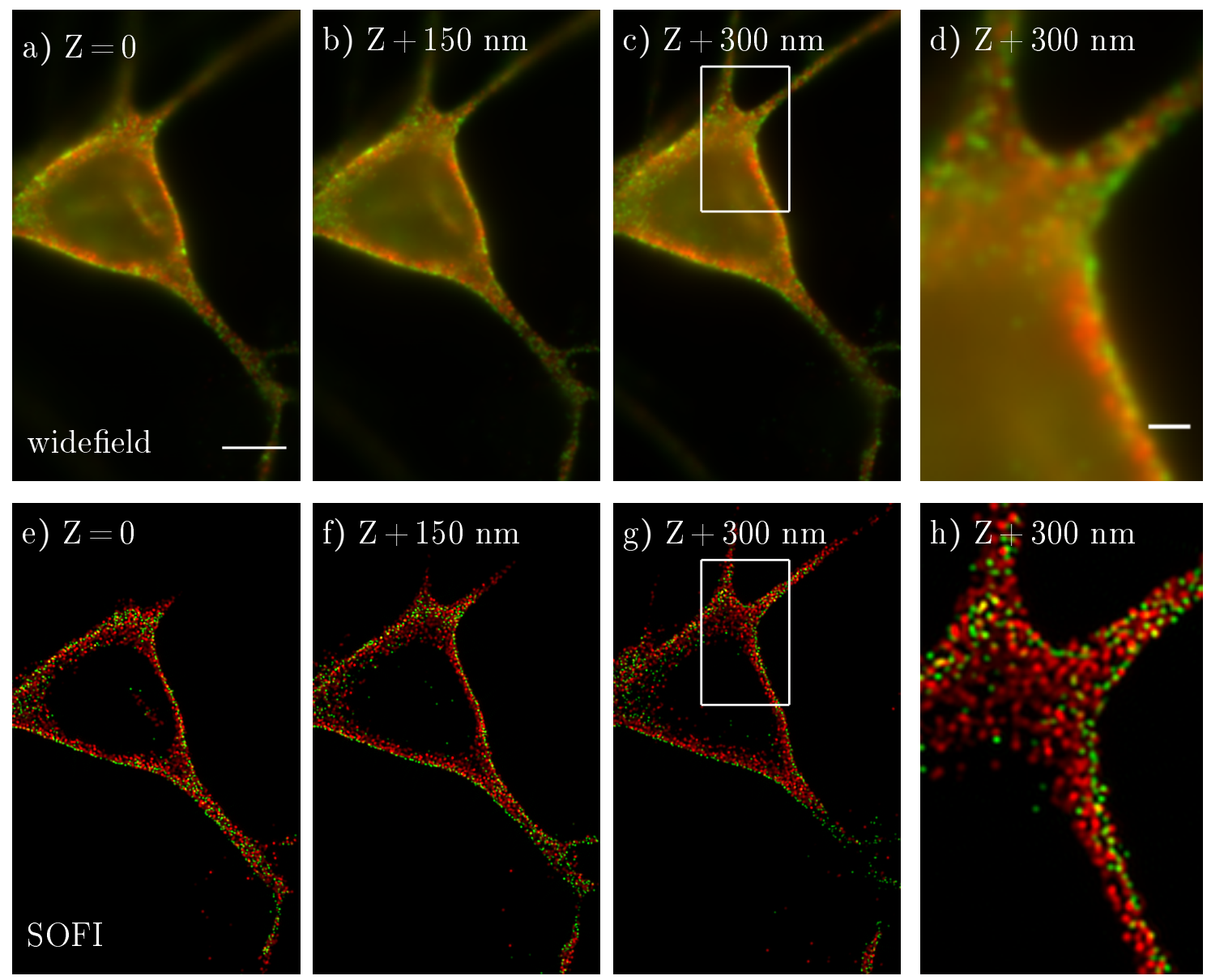

Figure 13. Widefield images of $G A B A_{B} R 1$ (red) and $G A B A_{B} R 2$ (green) of a neuron at axial distances of $150 \mathrm{~nm}(a, b, c)$, and corresponding SOFI images (e, f, g). Zoom-in of boxed area of widefield (d) and SOFI image (h). Scale bar: $5 \mu \mathrm{m}$, zoom-in $1 \mu \mathrm{m}$.

To gain information about the colocalization of the $\mathrm{GABA}_{\mathrm{B}} \mathrm{R} 1$ and $\mathrm{R} 2$ and elucidate their transport, data sets of rat hippocampal neurons were recorded. One data set consisted of movies from five to seven z-positions in two color channels. The lateral position was kept constant, while the axial position was increased by $150 \mathrm{~nm}$ between the images. Figure 13 compares the conventional dual-color widefield images of a neuron to the corresponding SOFI images. Three positions with an axial distance of $150 \mathrm{~nm}$ are shown. $\mathrm{GABA}_{\mathrm{B}} \mathrm{R} 1$ was labeled with $\mathrm{QD}_{605}$ and is depicted in red and $\mathrm{GABA}_{\mathrm{B}} \mathrm{R} 2$ was labeled with $\mathrm{QD}_{525}$ and is depicted in green.

While the widefield images all appear similar due to the fluorescence from areas other than the focal plane, the SOFI images show distinctly different slices of the neuron. This is due to the fact that the fluctuations of out-of-focus light are negligi- 
ble, and only fluctuating intensity is represented in the SOFI image. Furthermore, the resolution enhancement by a factor of 1.8 allows for a clear view of the distribution and relative locations of the two subunit types, which is especially clear in the zoom-in of the highest axial position (Fig. 13d and 13h).

Although the relatively simple widefield microscope used for data acquisition by itself does not provide any optical sectioning, it can be used to create z-stacks of neurons by application of SOFI. This is further illustrated by Fig. 14, where the widefield image corresponding to the first axial position of a stack is shown (Fig. 14a) together with the complete stack of SOFI images. The sections, recorded at an axial distance of $150 \mathrm{~nm}$, provide a clear view of the structure of the neuron and the $\mathrm{GABA}_{\mathrm{B}} \mathrm{R}$ subunit distribution.
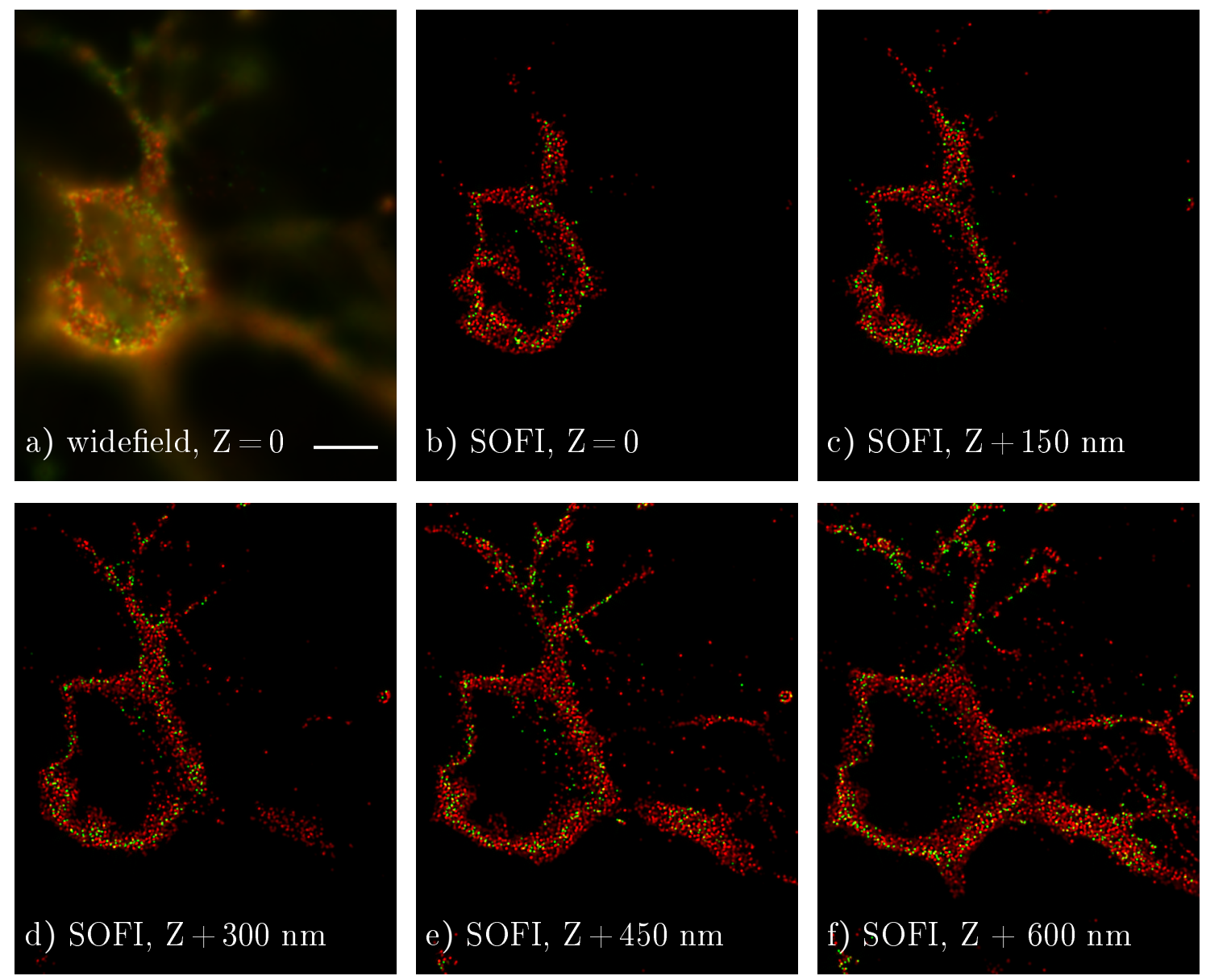

Figure 14. Complete SOFI z-stack. Widefield (a) and corresponding SOFI image (b) of $G A B A_{B} R 1$ (red) and $G A B A_{B} R 2$ (green) of a neuron, and subsequent SOFI sections (c, $d$, e, f) at axial distances of $150 \mathrm{~nm}$. Scale bar: $5 \mu \mathrm{m}$. 


\subsubsection{Discussion}

Widefield imaging does not provide optical sectioning, and almost the same fluorescence light is recorded at all z-positions. As a result, the widefield images of $\mathrm{GABA}_{\mathrm{B}}$ receptor subunits are almost identical for all z-positions. SOFI, in contrast, features increased resolution in all directions and excellent background suppression, allowing for z-sectioning on a widefield setup. Accordingly, the SOFI images from the different z-positions reveal different details of the neuron, such as different sections of the processes. An interesting detail of Fig. 13 is the excess of the $\mathrm{GABA}_{\mathrm{B}} \mathrm{R} 2$ receptor subunit, depicted in green, in the plasma membrane, while R1 is more abundant in the inner part of the soma and dendrite. While this distribution is not typical for all data sets, it is in accordance with the anchoring of unpaired $\mathrm{GABA}_{\mathrm{B}} \mathrm{R} 1$ to the ER membrane, while R2 has no such limitations.

Although a large number of high quality $3 \mathrm{D}$, dual-color SOFI data sets of GABA $\mathrm{B}_{\mathrm{B}}$ receptor subunits were recorded, obtaining enough information to clarify the transport of the $\mathrm{GABA}_{\mathrm{B}}$ receptor subunits proved ultimately impossible. This was due to the problematic labeling of the neurotransmitter receptor subunits. Against $\mathrm{GABA}_{\mathrm{B}} \mathrm{R} 1$ and $\mathrm{R} 2$, no primary antibodies existed, rendering direct immunostaining with QDots impossible. Therefore, in a first step, $\mathrm{GABA}_{\mathrm{B}} \mathrm{R} 1$ and $\mathrm{R} 2$ were tagged by transfection with epitopes against which primary antibodies are easily accessible. The desired labeling was achieved by subsequent immunostaining of the newly introduced epitopes. Transfection of the neurons, however, was unreliable, and transfection levels varied. As a consequence, not enough data could be collected from correctly labeled neurons. Additionally, not all possibility of doubt could be excluded whether measured differences in abundance of both subunits were due to peculiarities of the trafficking or of the transfection.

Nonetheless, the data sets demonstrate the high potential of SOFI as a valuable technique for biological applications, especially for samples with high background.

\subsubsection{Comparison of SOFI with confocal microscopy}

While the increase in resolution, the reduction of background fluorescence, and the optical sectioning provided by SOFI are obvious compared to widefield imaging, comparing second order SOFI to confocal microscopy can further assess its benefits.

To this end, the results of widefield, SOFI and confocal imaging of neuronal $\mathrm{GABA}_{\mathrm{B}} \mathrm{R}$ subunits were compared. The raw SOFI movie was recorded on a widefield 
setup, and the widefield image was calculated form the same movie by averaging over all individual frames, leading to identical positions of the widefield (Fig. 15a) and the SOFI image (Fig. 15b). The sample was then transfered to a confocal spinning disk setup, and a confocal image of the same neuron was recorded (Fig. 15c). Due to the setup change, the lateral and axial positions do not correspond exactly to those of the widefield and SOFI images.

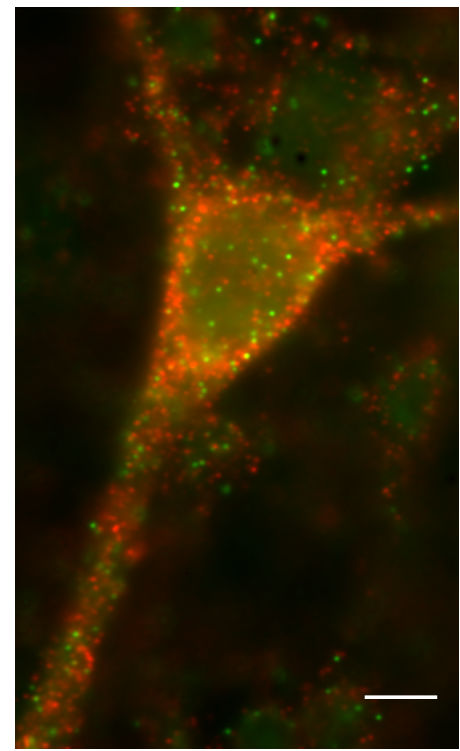

(a) widefield

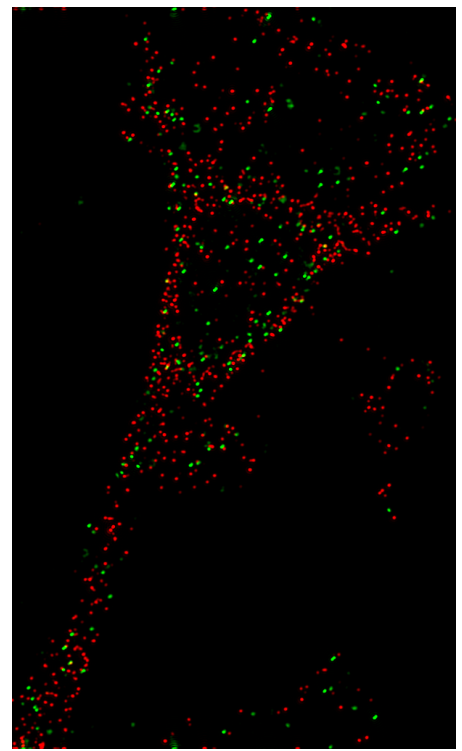

(b) SOFI

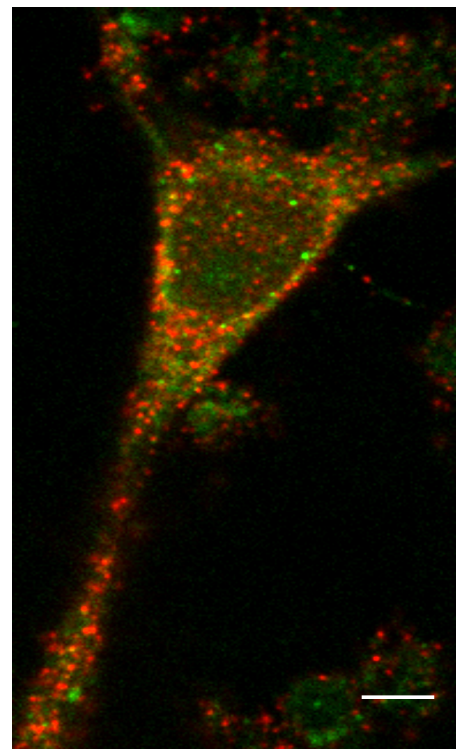

(c) confocal

Figure 15. Comparison of widefield (a), SOFI (b) and confocal spinning disk microscopy (c) on $G A B A_{B} R$ subunits of a neuron. The positions of the widefield and SOFI images are identical, while the position of the confocal image differs slightly. Scale bar: $5 \mu \mathrm{m}$.

\subsubsection{Discussion}

As expected, the widefield image is poorly resolved and heavily features out-offocus fluorescence. In comparison, images recorded on a confocal setup feature considerably less out-of-focus light, but the focal plane is still several hundreds of nanometers wide, typically 500 to $700 \mathrm{~nm}$. Consequently, while the background is reduced, all fluorescence within the focal plane is still detected.

SOFI, in contrast, reduces the PSF in $\mathrm{z}^{-}$as well in $\mathrm{x}$ - and $\mathrm{y}$-direction, but additionally omits all light whose intensity does not fluctuate, thus discarding of much more unwanted background fluorescence. As a result, SOFI images of QDotlabeled samples typically resemble clean cuts through the cell and show only the emitters in the focal plane. 


\subsection{Dependence of $\mathrm{Ca}^{2+}$ waves on distribution of ion channels}

\subsubsection{The inositol trisphosphate and ryanodine receptors}

Calcium ions $\left(\mathrm{Ca}^{2+}\right)$ play an important role in many functions of neurons, such as synaptic transmission and plasticity. One of the most versatile cellular signaling mechanisms is calcium release by inositol trisphosphate $\left(\mathrm{IP}_{3}\right)$ and ryanodine receptors. Both are large, homotetrameric, ligand-gated ion channels that reside in the ER membrane and interact functionally. They open upon binding an agonist and allow the release of calcium ions from the ER into the cytoplasm, thus propagating calcium waves 32 .

Functional interaction between the two receptor types suggests an influence of the relative localization of the receptors on the propagation of calcium waves. Consequently, disruption of the ER organization by transfecting neurons with mutated versions of the membrane resident structural ER protein atlastin-2 (ATL-2), and related alteration of the receptor distribution, would lead to a change of the calcium waves. To study this, my collaborators Omar Ramírez and colleagues from the University of Chile reproduced calcium waves in neurons by locally photo-releasing $\mathrm{IP}_{3}[33$ in wild type neurons and neurons with manipulated ER. They found an alteration of the calcium signals in the case of manipulated ER organization, possibly caused by a modified distribution of $\mathrm{IP}_{3}$ and Ryanodine receptors in the structurally changed ER. Until now, to our knowledge, no details are known about the relative localization of both receptor types in wild type or mutant neurons.

Dual-color SOFI images of $\mathrm{IP}_{3}$ and ryanodine receptors in neurons with different modifications of the ER allow us to link the altered calcium signals to the receptor distribution and colocalization. Therefore, their predominant isoforms in hippocampal neurons, $\mathrm{IP}_{3} \mathrm{R} 134$ and RyR2 ${ }^{35}$, were immunostained with QDots, and the ER was transfected with red fluorescent protein (RFP). The receptor distribution in neurons transfected with wild type ATL-2, neurons transfected with two different types of mutant ATL-2, and neurons without transfection were compared.

\subsubsection{D, dual-color SOFI of $\mathrm{IP}_{3} \mathrm{R} 1$ and RyR2}

To elucidate the relative distribution of $\mathrm{IP}_{3}$ receptors and ryanodine receptors in the ER and gain insight on their interactions, data sets of neurons with different ER structures were recorded. For SOFI imaging, $\mathrm{IP}_{3} \mathrm{R} 1$ and RyR2 were immunostained 

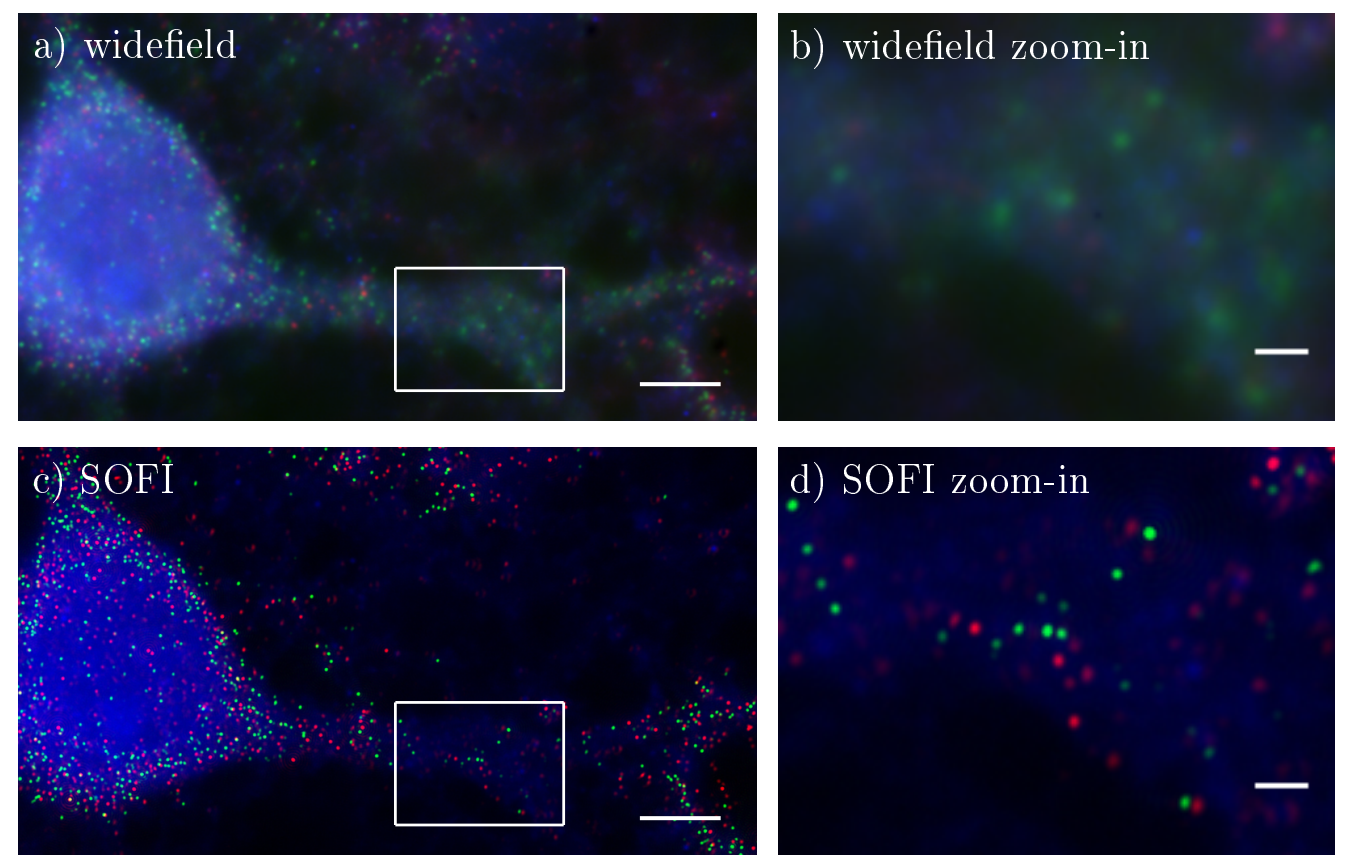

Figure 16. $I P_{3} R 1$ (red), RyR2 (green) and ER (blue) of a neuron with unmanipulated ER organization. Widefield (a) and SOFI image (c) of $I P_{3} R 1$ and RyR2 and corresponding zoom-in of boxed area $(b, d)$, with ER widefield images in all cases to provide context.

Scale bar: $5 \mu \mathrm{m}$, zoom-in $1 \mu \mathrm{m}$.

with QDots. Additionally, the ER marker KDEL was tagged with RFP to clarify the receptor positions in the context of the organelles of the cell. One data set consisted of five dual color images recorded at z-distances of $200 \mathrm{~nm}$. An example of a triple color image of a neuron with unmodified ER is given in Fig. $16\left(\mathrm{IP}_{3} \mathrm{R} 1\right.$ depicted in red, RyR2 in green and ER in blue). As conventional resolution is sufficient for the ER structure, the SOFI image is an overlay of the $\mathrm{IP}_{3} \mathrm{R} 1$ and RyR2 SOFI images and the ER widefield image. The combination of background elimination and the capability to resolve the individual emitters that tag the receptors allows a previously impossible view of the receptor distribution in the context of the ER.

For extensive studies of the distribution of the receptors over the volume of neuronal processes, z-stacks were recorded. Figure 17 compares the conventional widefield images of a neuron, transfected with mutated ATL-2, at three positions with an axial distance of $200 \mathrm{~nm}$ to the corresponding SOFI images. For clarity, the ER images are omitted. In contrast to the widefield images, which are similar for all z-positions due to out-of-focus light, the SOFI images show only a thin section of the neuron at each position. As a result, different structural details are visible in each individual SOFI image, greatly facilitating the analysis of the receptor distribution. 

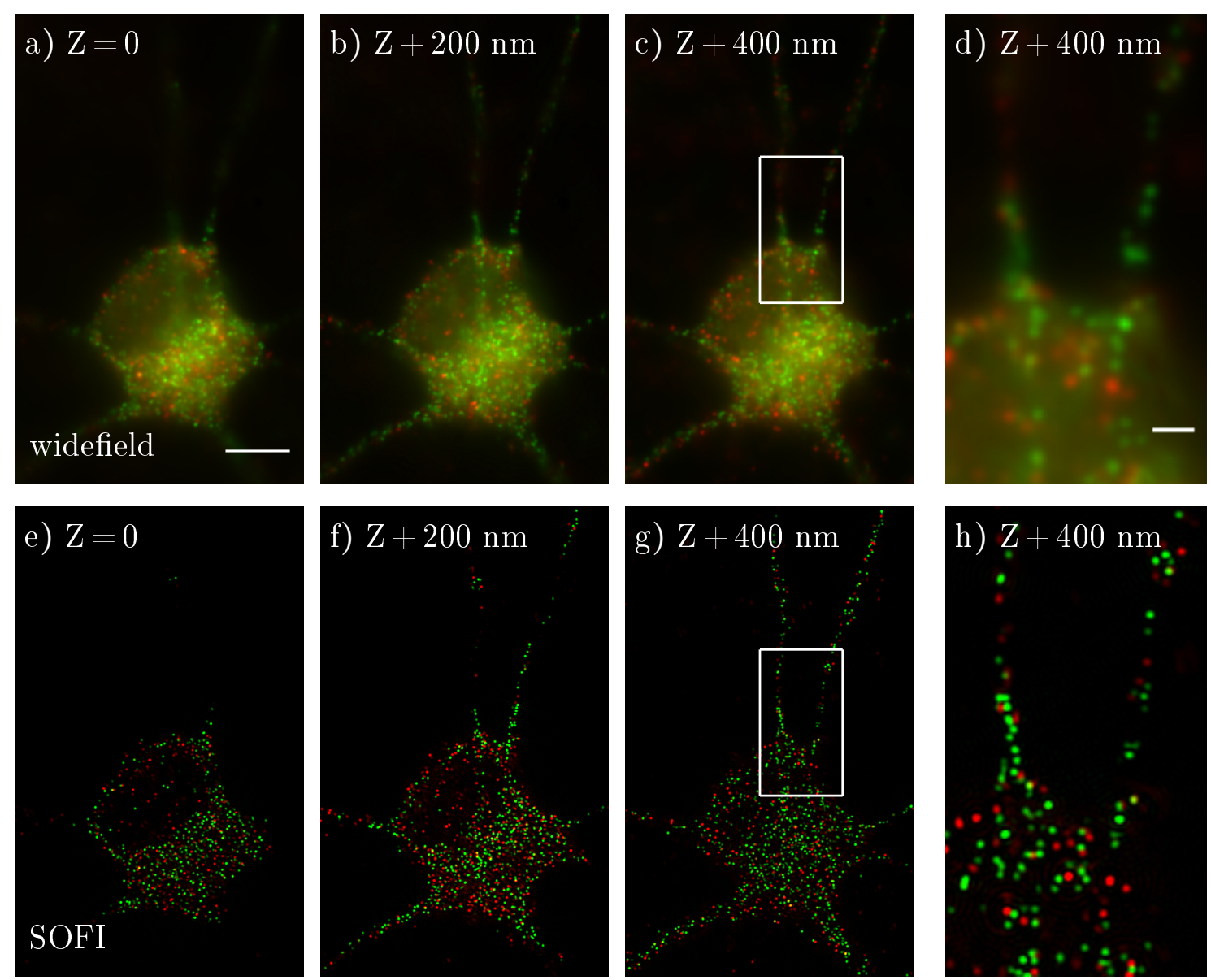

Figure 17. Widefield images of $I P_{3} R 1$ (red) and RyR2 (green) of a neuron at axial distances of $200 \mathrm{~nm}(a, b, c)$, and corresponding SOFI images $(e, f, g)$. Zoom-in of boxed area of widefield (d) and SOFI image (h). Scale bar: $5 \mu \mathrm{m}$, inset $1 \mu \mathrm{m}$.

\subsubsection{D capability of SOFI: axial receptor distribution}

To demonstrate the 3D capability of SOFI, a neuron was imaged over the large z-distance of $2.6 \mu \mathrm{m}$. A SOFI image was recorded every $200 \mathrm{~nm}$, and the individual images were subsequently calculated. To allow for an informative z-projection of the neuron, only the distribution of RyR2 is shown in Fig. 18 a, while corresponding dual-color widefield and SOFI images of the individual image at z-position $0.4 \mu \mathrm{m}$ are given in Fig. 18b and 18 .

\subsubsection{Discussion}

To study the distribution of $\mathrm{IP}_{3}$ receptors and ryanodine receptors in neuronal ER, 3D, dual-color SOFI data sets were acquired on a high number of individual neu- 

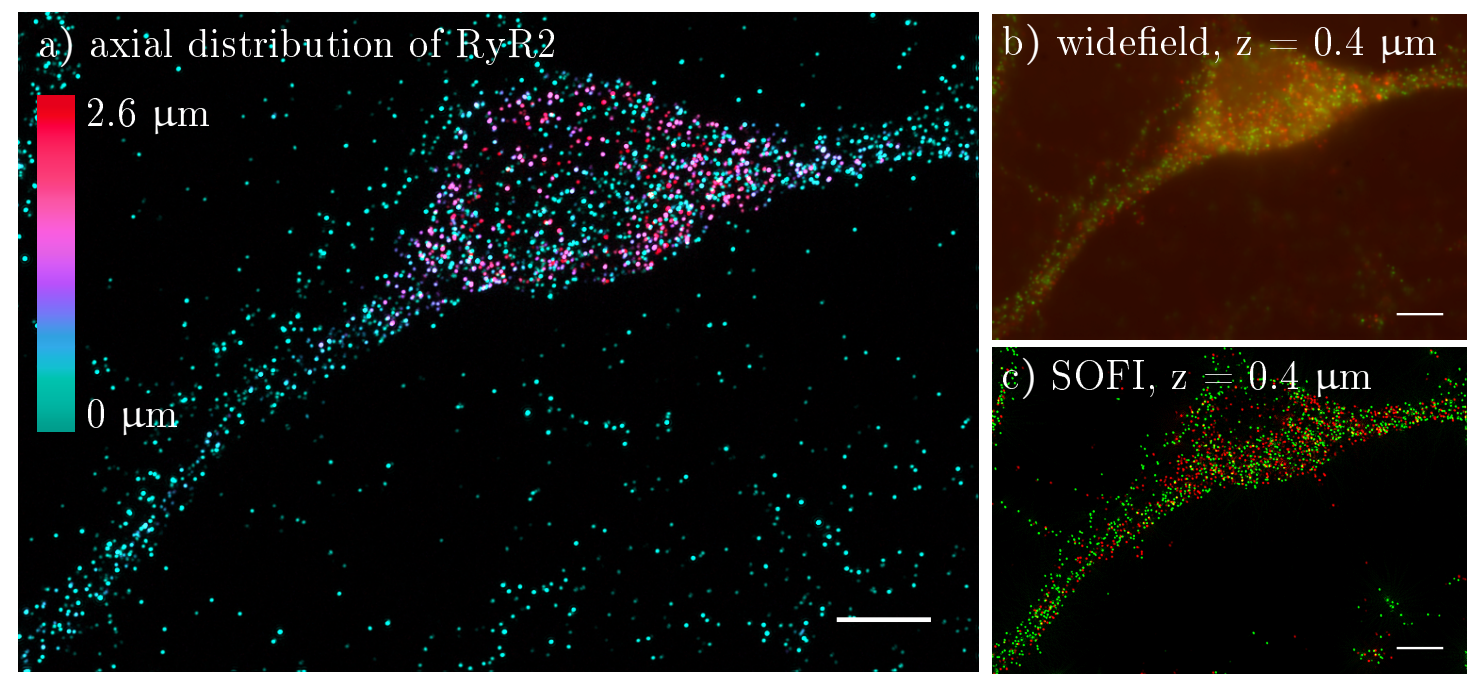

Figure 18. Axial distribution of the RyR2 receptors of a neuron over $2.6 \mu \mathrm{m}$ (a) reconstructed from SOFI images spaced every $200 \mathrm{~nm}$. Widefield (b) and SOFI (c) image of $R y R 2$ (green) and $I P_{3} R 1$ (red) at $z=0.4 \mu \mathrm{m}$. Scale bar: $5 \mu \mathrm{m}$.

rons. The distribution was studied on neurons transfected with wild type ATL-2, neurons transfected with two different types of mutant ATL-2, and neurons without transfection. SOFI provided highly promising superresolved images with next to no background fluorescence. These data sets, combined with the additional studies on the effect of ER disruption on calcium waves carried out by my collaborators, will allow us to elucidate the dependence of calcium signaling on ER organization and receptor distribution.

QDot, dual-color SOFI imaging over axial distances of 0.8 or $1 \mu \mathrm{m}$ has been used extensively. Interestingly, not even much larger axial distances pose any challenges to SOFI. Given sufficient setup stability, 3D sectioning at intervals of $200 \mathrm{~nm}$ over an axial distance of $2.6 \mu \mathrm{m}$ is easily implemented. This is made possible by the very low laser power required for SOFI imaging of QDots, which, combined with the high photobleaching stability of the labels, allows for prolonged imaging of large sample areas. Thus, not only clear z-sections, but a 3D image of a whole neuron in z-direction can be recorded (Fig. 18). 


\section{SOFI with reversibly photoswitchable fluorescent proteins}

\subsection{Reversibly photoswitchable fluorescent proteins}

Fluorescent proteins (FPs) are genetically encoded fluorophores based on proteins responsible for bioluminescence in certain species of jellyfish, corals and other marine animals. By means of molecular biology, the gene encoding the FP can be introduced into organisms ${ }^{36}$, and FPs can be fused to any desired protein. As a result, fluorophores are expressed and linked to the structure of interest by the studied organism instead of introduced from the outside. The availability of genetically encoded labels allows for monitoring spatial distribution and temporal dynamics in live cells, and thus to link structure to function. Even when not monitoring dynamics, there is less risk of mislabeling or altered structures compared to immunostaining with organic dyes, which is typically performed in combination with cell fixation. In addition to fluorescence microscopy, FPs are frequently used as a reporter for the expression of other genes, and many variants have been developed for a large variety of applications 37 .

A fluorescent protein generally consists of an 11-stranded $\beta$-barrel with a central helix containing the chromophore. The chromophore typically maturates from a chain of three amino acids under autocatalysis aided by residues of the barrel. Properties like quantum yield, absorption and emission maxima, and folding efficiency at specific temperatures (p. ex. $37^{\circ} \mathrm{C}$ for applications in mammalian cells) depend on the structure of the chromophore as well as the $\beta$-barrel. The same chromophore in $\beta$-barrels with different residues can even lead to FPs with fundamentally different properties. Therefore, changing one or more amino acids that form the chromophore or the surrounding structure by mutagenesis can have a large influence on the properties of the fluorescent protein 38 . Since the discovery of the green fluorescent protein (GFP) [39]40 and its first applications to biological research 41, many variants have been developed by mutagenesis. These include GFPs with improved quantum yield, folding efficiency and absorption maximum (p. ex. enhanced green fluorescent protein (EGFP) 42$]$, and fluorescent proteins with different absorption and emission maxima (p. ex. mRFP 43$]$, mCherry [44]).

A further development are phototransformable FPs, which undergo transitions between different fluorescent and non-fluorescent states upon irradiation. Subtypes 
of phototransformable FPs are photoactivatable FPs, which change from a dark to a fluorescent state, photoconvertible FPs, which change fluorescence wavelength, and photoswitchable FPs (RSFPs), which reversibly switch between a dark and a fluorescent state. Among many other applications, phototransformable FPs enable several important techniques in the field of superresolution microscopy.

For instance, the fabrication of photoactivatable and photoconvertible FPs ( $p$. ex. EosFP 45] was crucial for the development of photoactivated localization microscopy (PALM) 1314146. Stochastically transferring a small subset of FPs to a state fluorescent at a certain wavelength (by photoactivation or photoconversion) allows for the localization of this subset. Over the course of the data acquisition, additional FPs are activated or converted and then localized, while others are rendered dark due to photobleaching. Thus, all FPs can be localized and a complete superresolved image can be reconstructed. While PALM can be performed using photoactivatable FPs, photoconvertible FPs are more convenient. Since they are in fluorescent states of different wavelengths before and after conversion, the first emission wavelength can be used to choose a suitable sample position and the successive switching to the other for localization. Superresolution by RESOLFT microscopy $\sqrt{8}$ is based on reversible saturable optical fluorescence transitions between different states of emitters. For example, transitions between a fluorescent state of fluorophore and a dark state caused by stimulated emission can be used, or between the fluorescent and dark states of photoswitchable FPs. For RESOLFT microscopy with photoswitchable FPs, only low irradiation intensities are necessary, reducing the risk of light-induced damages.

Fluorescent proteins that undergo transitions between a fluorescent and a nonfluorescent state upon irradiation (reversibly photoswitchable fluorescent proteins (RSFPs)) offer a wide range of applications as research tools. Consequently, since the first observation of photoswitching in proteins (in YFP [47]) and the first design of such proteins (p. ex. KFP [48, Dronpa ${ }^{[9]}$ ), these labels have been developed into a large library of RSFPs 50151 . Many RSFPs with improved properties are now available, such as enhanced resistance against switching fatigue, higher brightness and different switching properties for many different applications. The photoswitching is typically based on cis-trans isomerization of the chromophore and follows one of three mechanisms 50 . In the cases of negative and positive switching, the wavelength that elicits fluorescence transfers the fluorophore into the off-state or on-state, respectively, and the fluorophore is switched back by another wavelength. Dreiklang, aptly named after the German word for a harmonic triad of three notes in music, 
is a special case 52 . Here, switching is decoupled from the illumination absorbed in the on-state. On-switching, off-switching and fluorescence are therefore induced by three distinct wavelengths.

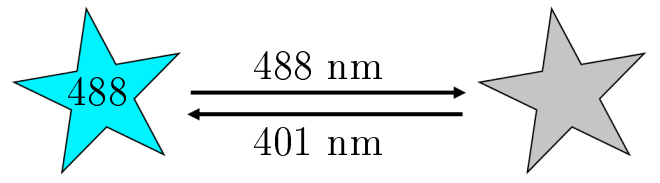

(a) rsEGFP and related green FPs with negative switching are switched by $488 \mathrm{~nm}$ and $401 \mathrm{~nm}$ light. Fluorescence is elicited at $488 \mathrm{~nm}$.

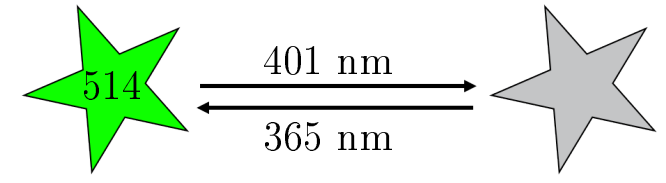

(b) Dreiklang is switched by $401 \mathrm{~nm}$ and $365 \mathrm{~nm}$ light. Fluorescence is elicited at $514 \mathrm{~nm}$.

Figure 19. Illustration of negative and decoupled switching. FPs with negative switching are rendered dark by the wavelength responsible for fluorescence (a). Dreiklang photoswitching is decoupled from fluorescence read-out (b).

Fig. 19 illustrates the dependence of the photoswitching on the irradiation wavelengths. Reversibly photoswitchable enhanced green fluorescent protein (rsEGFP) [53] and related green FPs with negative switching are transfered to their non-fluorescent state upon irradiation at $\sim 488 \mathrm{~nm}$, which doubles as the fluorescence read-out wavelength. Fluorescence recovery is promoted with irradiation at $401 \mathrm{~nm}$. Consequently, continued fluorescence excitation without irradiation at $401 \mathrm{~nm}$ only elicits fluorescence light for a brief period of time, until all proteins have been transfered to the non-fluorescent state. Dreiklang fluoresces upon excitation at $514 \mathrm{~nm}$, is switched to a dark state by $401 \mathrm{~nm}$ light, and switched back to the fluorescent state by 365 nm light. Dreiklang thus can be imaged continuously with $514 \mathrm{~nm}$, and off- and on-switching by light of the respective wavelength is completely separate from the fluorescence read-out.

Since the properties of a fluorescent protein depend on the chromophore itself as well as its interaction with the side chains through multiple stabilizing noncovalent interactions, the residues determine, among other things, the protonation state, polarization, spatial conformation, and rotational freedom. Side chains that keep the chromophore rigidly in place prevent phototransformation, while phototransformation is enabled by residues that facilitate rearrangements and isomerization. Hence, changing the residues by mutagenesis can dramatically alter the photoswitching behavior of an FP.

For proteins with negative switching behavior, studies support cis-trans isomeri- 
sation of the chromophore, concomitant to or followed by protonation, as underlying molecular mechanism of the off-switching [50|5455. Typically, absorption spectra of the protein in the on-state contain a band around 480 - $490 \mathrm{~nm}$, corresponding to the cis-configuration, which is replaced by a band around $400 \mathrm{~nm}$, corresponding to the trans-configuration, upon photoswitching to the off-state. To verify this mechanistic view, mutant FPs with faster isomerisation and photoswitching were created by removing bulky and obstructing side chains in the chromophore pocket of Dronpa, facilitating the cis-trans isomerisation. The RSFPs developed as a consequence are rsFastLime 56 , where an exchange of the small glycine for the bulky valin removed steric hindrance and increased the switching speed by a factor of 50, and Dronpa(M159T) 56 with over 1000-fold increase in switching speed due to the exchange of threonine for methionine, but poorer quantum yield $\Phi_{F l}$ (0.85 for Dronpa, 0.77 for rsFastLime and 0.23 for Dronpa(M159T)). Similarly, EGFP was used as a starting point for the development of fluorescent proteins with negative photoswitching. Examples are rsEGFP [53, rsEGFP2 [57 and rsEGFP(N205S) 58. Of these proteins, the off-switching of rsEGFP2 is the fastest, followed by rsEGFP. The photoswitching of rsEGFP(N205S) is slower, although not as slow as Dronpa's. rsEGFP(N205S) emits about twice the number of photons per cycle than rsEGFP and rsEGFP2 (which have quantum yields of 0.36 and 0.3 ), rendering it a very interesting label.

Dreiklang 52 was developed from GFP mutant Citrine, which had shown a small receptivity for fluorescence modulation, by introducing four mutations. Among other effects, these mutations increased the $\mathrm{pK}_{\mathrm{a}}$ of the chromophore and thereby decreased the susceptibility to protonation. In the on-state, the autocatalytically formed chromophore consists of two largely coplanar rings over which spans the $\pi$-system responsible for fluorescence. It exists in a protonated and an unprotonated form, resulting in two absorption bands with maxima at $412 \mathrm{~nm}$ and $511 \mathrm{~nm}$. Irradiation at the $511 \mathrm{~nm}$ band induces fluorescence, while irradiation at $412 \mathrm{~nm}$ induces off-switching by hydration of one of the rings. Hydration shortens the $\pi$ system which causes the maximum of the absorption to shift to $340 \mathrm{~nm}$. Irradiation at this absorption band leads to on-switching by dehydration of the chromophore. The decoupling of the switching from the fluorescence and the consequent control over the switching speeds via irradiation intensities make Dreiklang a versatile fluorescent label, as demonstrated by its use for localization techniques as well as for RESOLFT 52159.

Induction of suitable statistically independent intensity fluctuations in RSFPs 
allows for the expansion of SOFI to live cell imaging. The random blinking of Dronpa at high read-out intensities has already been used for SOFI imaging 60 , and the variant pcDronpa2 was especially designed for combined SOFI and STORM imaging 61. However, while almost every type of blinking can be exploited, best SOFI results are achieved if the blinking fulfills certain conditions. Moreover, live cell imaging requires fast data acquisition, and SOFI imaging is fastest when the blinking conditions are met. Therefore, the ability to tune the photoswitching behavior is desirable. Such control of the duration of the on- and off-states of RSFPs, while ensuring that the transitions remain statistically independent, can be achieved by a suitable choice of the irradiation intensities. rsEGFP [53], rsEGFP2 [57, Dronpa(M159T) [56, rsFastLime [56, and rsEGFP(N205S) 58, which cover a large variety of negative switching kinetics, as well as Dreiklang [52] were chosen as potential SOFI labels.

\subsection{Principle of photoswitching control}

\subsubsection{Inducing intensity fluctuations}

Since switching from the fluorescent to the non-fluorescent state and back depends on irradiation by light of certain wavelengths, it should be possible to control the switching by controlling the intensity of the light. Photoswitching control can thus be achieved by continuous irradiation at specific intensities of the off-switching wavelength, the on-switching wavelength and the wavelength for fluorescence readout. These are three distinct wavelengths for Dreiklang, while off-switching and readout wavelength are the same for the proteins with negative photoswitching.

Continuous irradiation of the proteins with negative switching with $488 \mathrm{~nm}$ light elicits fluorescence, but also switches the proteins to a dark-state. The off-switching can be counterbalanced by on-switching through additional continuous irradiation with light of $401 \mathrm{~nm}$, otherwise fluorescence vanishes. For conventional imaging with RSFPs with negative switching behavior, both wavelengths are used at high intensities. This leads to very fast on-switching, negligible duration of the off-state, and therefore quasi-continuous fluorescence from each individual protein. By reducing the irradiation intensity at $488 \mathrm{~nm}$, the duration of the on-state can be extended, while the average number of emitted photons per on-state remains unchanged. Similarly, reducing the intensity at $401 \mathrm{~nm}$ extends the off-state. Consequently, by adjusting the intensities for both wavelengths, intensity fluctuations can be induced. 
The decoupled switching of Dreiklang differs, as the fluorescence readout irradiation at $514 \mathrm{~nm}$ is independent of the switching. As a result, the irradiation intensity at $514 \mathrm{~nm}$ can be chosen more freely, while the intensities of the on- and off-switching wavelengths (365 nm and $401 \mathrm{~nm}$, respectively) are adjusted to control the blinking. Although the absorption maximum of the Dreiklang on-switching lies at $365 \mathrm{~nm}$, irradiation at $375 \mathrm{~nm}$ is almost as effective and was used for on-switching for all experiments.

\subsubsection{Ensemble studies of photoswitching kinetics}

As a first step towards SOFI with RSFPs, the irradiation intensity combinations that generate the optimal blinking had to be identified. To this end, the lifetimes of the on- and off-states ( $\tau_{\text {off }}$ and $\tau_{o n}$, respectively) were extracted from fluorescence time traces of protein ensembles. The time traces were measured by irradiating E.coli filled with the fluorescent protein continuously with the fluorescence readout light and periodically with the photoswitching wavelengths. Typically, time traces were recorded from three to four E.coli per condition, each with multiple switching events, over 25 to $75 \mathrm{~s}$ at an exposure time of $2.5 \mathrm{~ms}$ to ensure a high temporal resolution. Time traces were calculated by subtracting the time-dependent average background from the time-dependent average intensity of the bacteria fluorescence and correcting for bleaching. Then, exponential fits were performed to extract the half lifes of the decay and rise, which serve as a measure for the lifetimes of the states.

\subsubsection{Fluorescent proteins with negative photoswitching}

To record time traces of proteins with negative photoswitching, samples were continuously irradiated at a constant $488 \mathrm{~nm}$ intensity. Irradiation at $401 \mathrm{~nm}$ was added for periods of several seconds. An exemplary measured time trace from the protein rsEGFP(N205S) with a schematic of the irradiation is shown in Fig. 20. A high fluorescence signal was recorded during irradiation with both wavelengths. Upon removal of $401 \mathrm{~nm}$ irradiation, the fluorescence decayed exponentially to a constant background level. Fitting of this exponential decay curve yielded $\tau_{\text {fall }}$ which is identical to $\tau_{o f f}$, the half life of the on-state, and depends on the irradiation intensity at $488 \mathrm{~nm}$. Upon renewed irradiation at $401 \mathrm{~nm}$, the fluorescence intensity rose exponentially to its former value. An exponential fit of this rise yielded $\tau_{\text {rise }}$. During the 


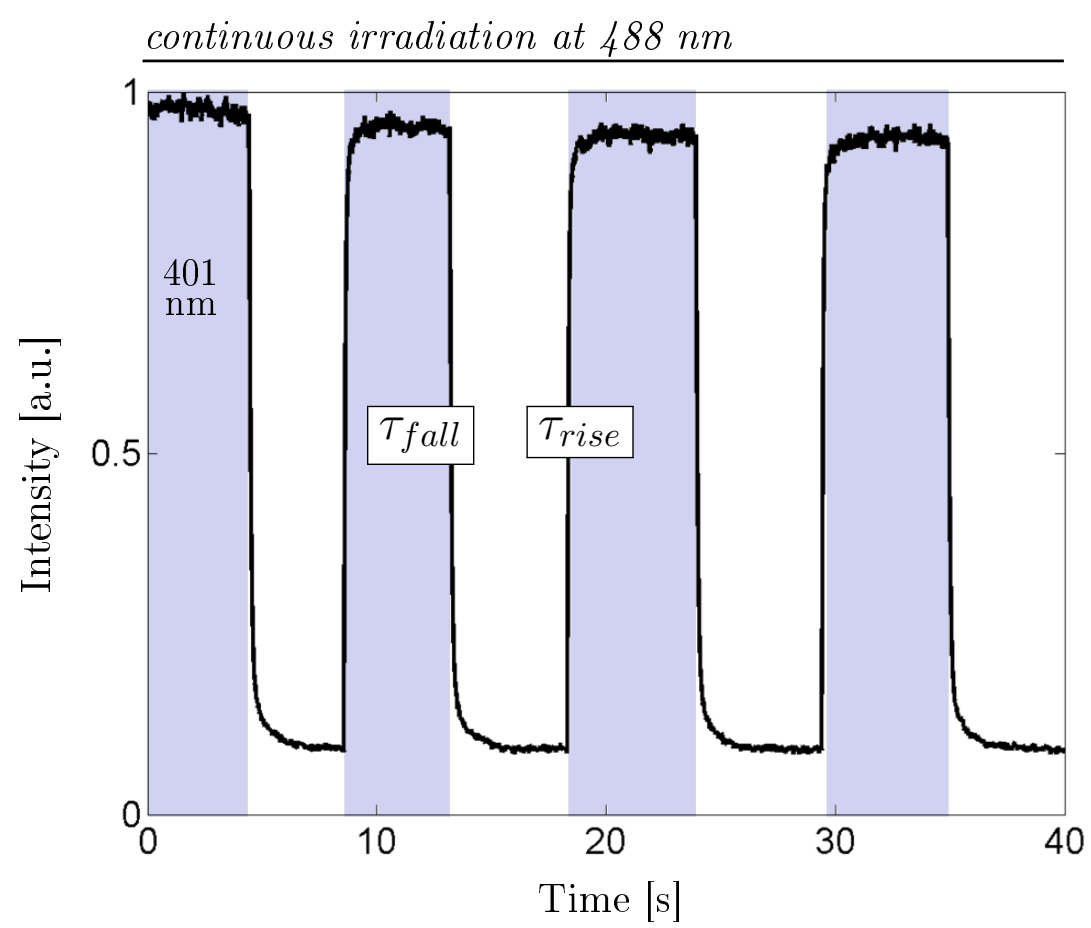

Figure 20. Example of a time trace of rsEGFP(N205S), a protein exhibiting negative photoswitching. An ensemble of the protein is continuously irradiated at $488 \mathrm{~nm}$, and periodically at $401 \mathrm{~nm}$. $\tau_{\text {off }}$ and $\tau_{\text {on }}$ can thus be determined.

rise of the fluorescence signal, two competing processes take place: on-switching by $401 \mathrm{~nm}$ and off-switching by $488 \mathrm{~nm}$. As a result, $\tau_{\text {rise }}$ depends on the intensity of both wavelengths. $\tau_{\text {on }}$, the lifetime of the off-state, can be calculated from $\tau_{\text {fall }}$ and $\tau_{\text {rise }}$, according to

$$
1 / \tau_{\text {on }}=1 / \tau_{\text {rise }}-1 / \tau_{\text {fall }}
$$

or, in rate constants,

$$
k_{\text {on }}=k_{\text {rise }}-k_{\text {fall }} \text {. }
$$

$\tau_{\text {on }}$ then depends only on the intensity of the irradiation at $401 \mathrm{~nm}$.

Although this approach is reasonably suitable for the low irradiation intensities used, it is a simplification of the behavior of RSFPs with negative photoswitching. Apart from the main off- and on-state, it is possible that intermediate states play a role in the switching, leading to multiexponential decay curves. Additionally, crosstalk and thermal relaxation from the dark state can cause extra photoswitching. Bleaching is likely at simultaneous irradiation with the on- and off-switching light, and although we corrected for it, bleaching artifacts may still be present. 


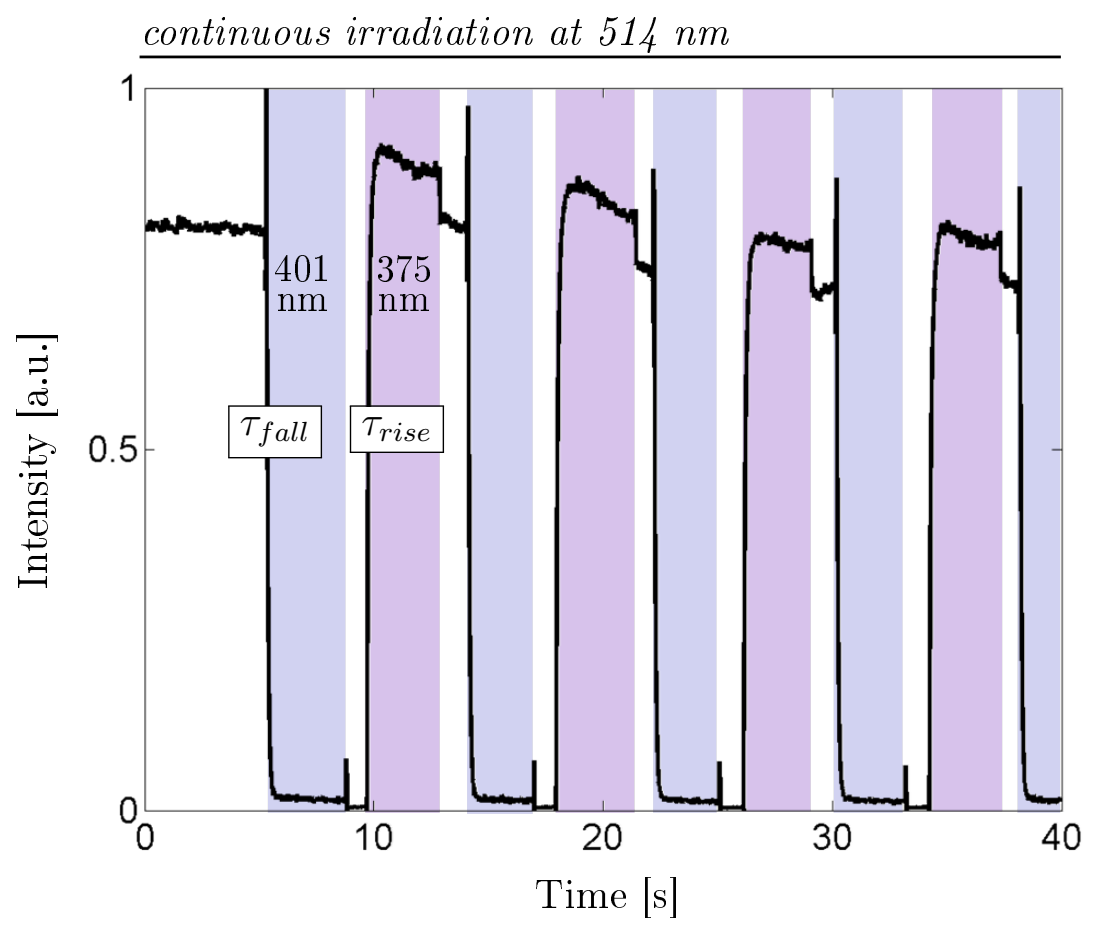

Figure 21. Example of a time trace of Dreiklang, a protein exhibiting decoupled photoswitching. An ensemble of the protein is continuously irradiated with light of $514 \mathrm{~nm}$ to elicit fluorescence. Irradiation at $401 \mathrm{~nm}$ promotes off-switching, while irradiation at 375

$\mathrm{nm}$ promotes on-switching. From these time traces, $\tau_{\text {off }}$ and $\tau_{\text {on }}$ can be calculated.

\subsubsection{Fluorescent proteins with decoupled photoswitching}

Since Dreiklang's switching is decoupled from the fluorescence readout, the read-out wavelength $(514 \mathrm{~nm})$ can be used at any intensity, while the switching is controlled using two additional wavelengths. The recording of a time trace with Dreiklang therefore consisted of constant irradiation at $514 \mathrm{~nm}$, and periodic, alternating irradiation at $401 \mathrm{~nm}$ and $375 \mathrm{~nm}$.

Figure 21 shows an example of a time trace with a schematic of the irradiation. As soon as irradiation at $401 \mathrm{~nm}$ was added, exponential decay of the fluorescence intensity to a constant background set in, yielding the lifetime of the on-state $\tau_{\text {fall }}$. It depends only on the intensity of $401 \mathrm{~nm}$ irradiation and was therefore identical to $\tau_{\text {off }}$. Stopping irradiation at $401 \mathrm{~nm}$ had negligible influence on the fluorescence, while irradiating at $375 \mathrm{~nm}$ led to an exponential rise to complete fluorescence recovery. The exponential fit yielded the lifetime of the off-state, $\tau_{\text {rise }}$. In the case of Dreiklang, $\tau_{\text {rise }}$ is identical to $\tau_{o n}$ and depends only on the intensity of $375 \mathrm{~nm}$. An overview of the different lifetimes $\tau$ as a measure for the duration of on- and off-states for both types of photoswitching kinetics is given in Table 2, As is the case 
for the proteins with negative switching, this approach is based on a simplification of the behavior of Dreiklang at low irradiation intensities.

\begin{tabular}{c||c|c} 
& negative switching & decoupled switching \\
\hline \hline$\tau_{\text {off }}$ & $\tau_{\text {fall }}$ & $\tau_{\text {fall }}$ \\
\hline$\tau_{\text {on }}$ & $1 / \tau_{\text {rise }}-1 / \tau_{\text {fall }}$ & $\tau_{\text {rise }}$
\end{tabular}

Table 2. Relation of lifetimes of the on- and off-states ( $\tau_{\text {off }}$ and $\left.\tau_{\text {on }}\right)$ to lifetimes extracted from time traces ( $\tau_{\text {fall }}$ and $\left.\tau_{\text {rise }}\right)$, for proteins with negative and decoupled photoswitching.

\subsubsection{Lifetimes determined from time traces}

The lifetimes of the off- and on-states $\left(\tau_{\text {on }}\right.$ and $\left.\tau_{\text {off }}\right)$ depend strongly on irradiation intensities, as can be seen in the comparison of two time traces of rsEGFP(N205S) in Fig. 22 recorded under laser excitation. For both time traces, the intensity of 401 $\mathrm{nm}$ was kept constant, resulting in the very similar values for $\tau_{\text {on }}$ of $251 \mathrm{~ms}$ (Fig. 22a and $258 \mathrm{~ms}$ (Fig. 22b), as well as an identical appearance of the fluorescence intensity increase. The intensity at $488 \mathrm{~nm}$ used for Fig. 22a, in contrast, was only a fifth of that used for Fig. 22b. This resulted in slower off-switching in the first case (Fig. 22a), with a $\tau_{\text {off }}$ of $1833 \mathrm{~ms}$, compared to $221 \mathrm{~ms}$ for the second case (Fig. 22b).

Lifetime results were consistent for the E.coli measured at a specific intensity setting and measurement occasion. When repeating experiments with identical samples and settings on a different occasion, however, results almost always differed from the previous measurements. For example, the lifetimes of rsEGFP(N205S) at $10 \% 401$ $\mathrm{nm}$ and $50 \% 488 \mathrm{~nm}$ laser intensity were $\tau_{\text {off }}=141 \pm 10 \mathrm{~ms}$ and $\tau_{\text {on }}=13 \pm 1$ ms, measured on three bacteria, each with multiple switching events. On a later occasion, the same settings yielded the reduced lifetimes of $\tau_{\text {off }}=47 \mathrm{~ms}$ and $\tau_{\text {on }}=$ $10 \mathrm{~ms}$ in a control experiment on one bacterium. Likewise, Dreiklang lifetimes at $100 \% 401$ and $375 \mathrm{~nm}$ and $20 \% 514 \mathrm{~nm}$ laser intensity were $\tau_{\text {off }}=154 \pm 12 \mathrm{~ms}$ and $\tau_{o n}=196 \pm 12 \mathrm{~ms}$ in the first measurements, but were shortened to $\tau_{\text {off }}=76$ $\mathrm{ms}$ and $\tau_{\text {on }}=67 \mathrm{~ms}$ in the experiment on the second occasion.

Since heavily used setups are re-adjusted frequently, a likely reason for these deviations is a slightly different adjustment of the light source that alters the surface power density on the sample. Although the laser adjustment seemed identical for all measurements, small, unavoidable differences changed the photoswitching speed 


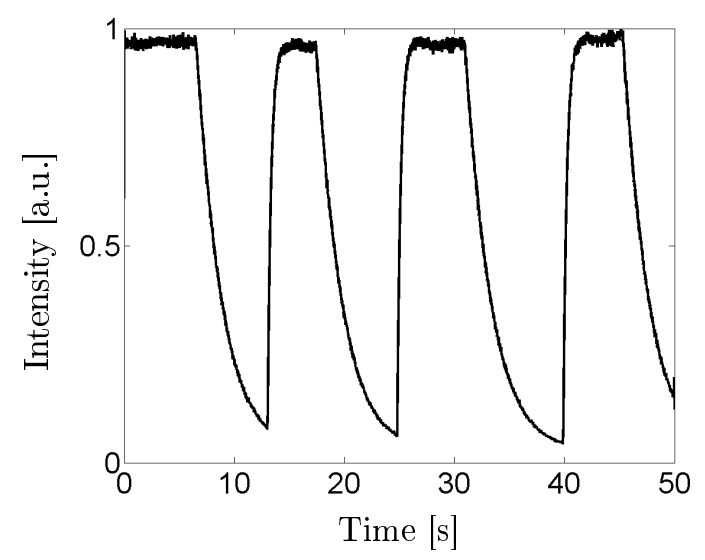

(a) $5 \% 488 \mathrm{~nm}, 1 \% 401 \mathrm{~nm}$ $\tau_{\text {off }}=1833 \mathrm{~ms}, \tau_{\text {on }}=251 \mathrm{~ms}$

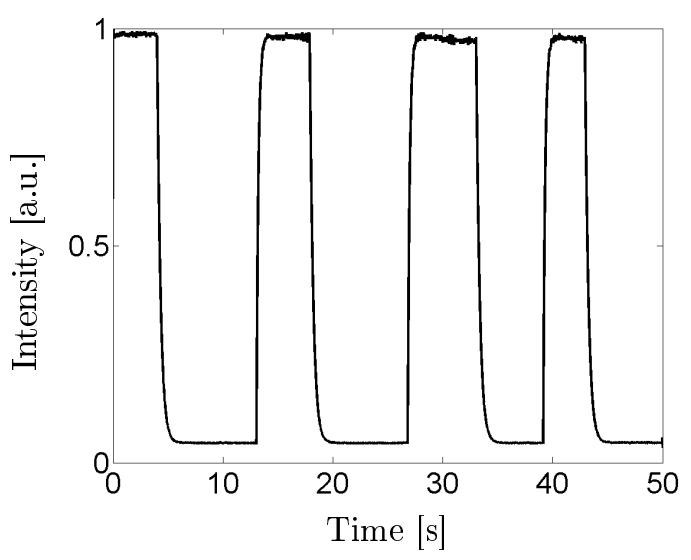

(b) $25 \% 488 \mathrm{~nm}, 1 \% 401 \mathrm{~nm}$ $\tau_{\text {off }}=221 \mathrm{~ms}, \tau_{\text {on }}=258 \mathrm{~ms}$

Figure 22. Comparison of time traces of rsEGFP(N205S) at different irradiation intensity combinations. $401 \mathrm{~nm}$ irradiation intensity was identical for both curves, resulting in similar lifetimes of the off-state $\left(\tau_{o n}\right)$. Off-switching was faster and $\tau_{\text {off } f}$ shorter in $(b)$, due to the higher intensity of $488 \mathrm{~nm}$ irradiation.

noticeably. In this particular case, all lasers in question apparently were better aligned on the second occasion, leading to higher surface power density and faster photoswitching. This can not be verified directly, as the error of measurements of the surface power density greatly exceeds the deviations due to slight differences in alignment. As a consequence, only measurements from the same measurement occasion are compared.

\subsubsection{Influence of irradiation intensity on photoswitching}

The dependences of photoswitching on irradiation intensities are illustrated in plots of the lifetimes against the intensity of the off-switching light. An estimation for such a dependence plot is shown in Fig. 23. Although the photoswitching of proteins with negative and decoupled switching differs, their dependency of the lifetimes of the on- and off-states on the intensities of the irradiation used for switching is similar.

$\tau_{\text {on }}$ depends on the intensity of the on-switching light. Consequently, the curve is expected to be roughly parallel to the x-axis, at a shorter lifetime for a higher intensity of the on-switching light and at a longer lifetime for a lower intensity. Since $\tau_{\text {off }}$ can be considered independent of the on-switching light, curves for higher and lower on-switching intensity in theory are identical and decrease exponentially with increasing intensity of the off-switching wavelength. 
For proteins with negative switching, $401 \mathrm{~nm}$ is the on-, and $488 \mathrm{~nm}$ is the offswitching light, whereas for Dreiklang with its decoupled switching $375 \mathrm{~nm}$ is the on-, and $401 \mathrm{~nm}$ is the off-switching light. For Dreiklang, the intensity at $514 \mathrm{~nm}$ was kept constant for each dependency plot. Theoretically, from such a plot, the ideal intensities for the desired switching can be chosen. However, additional effects complicated the choice of parameters.

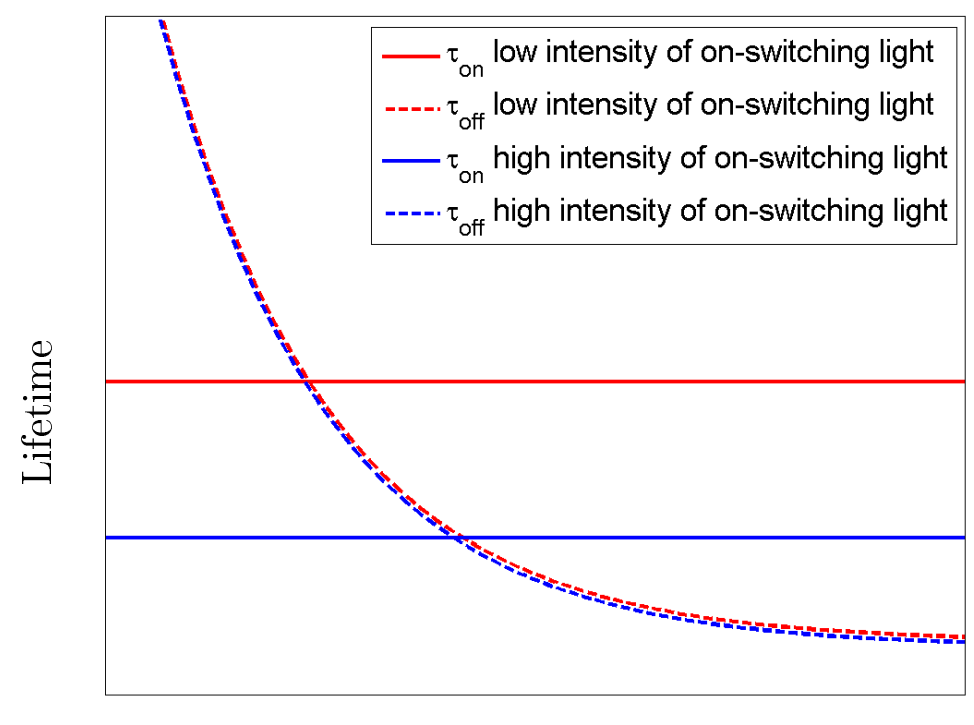

Intensity of off-switching light

Figure 23. Scheme of the idealized estimated dependence of lifetimes on irradiation intensities. The intensity of the on- and off-switching light determines $\tau_{\text {on }}$ and $\tau_{\text {off }}$, respectively.

\subsection{Determination of photoswitching}

To identify the proteins best suited for SOFI, the different photoswitching kinetics of several RSFPs were studied. Furthermore, knowledge about the dependence of photoswitching behavior on irradiation intensities allows for the control of the intensity fluctuations. The studies consequently cover the lifetime ranges most interesting for SOFI. From the proteins with negative switching we chose Dronpa(M159T), rsFastLime, rsEGFP, rsEGFP2, and rsEGFP(N205S) for the experiments. Additionally, the potential of the decoupled switching of Dreiklang for SOFI was studied. Two widefield setups, one equipped with lasers (see 7.2.2) and one with LEDs (see 7.2.1), were used for the evaluation of the proteins with negative photoswitching. On the LED setup, two different wavelengths for the combined off-switching and fluorescence readout were studied, leading to a total of three different setup options. 
For Dreiklang, the widefield setup equipped with lasers was used.

Photoswitching control proved to be most reliable for rsEGFP(N205S) and Dreiklang, making them the RSFPs most promising for imaging. Therefore, this section focuses on their photoswitching. The photoswitching kinetics of rsEGFP, rsEGFP2, Dronpa(M159T), and rsFastLime are presented as supplementary data in section 8.

Note that the irradiation intensities are given relative to total laser or LED power due to the error-prone nature of the measurements of sample surface power density. Table 3 (section 7) links total power to approximate surface power density. Furthermore, lifetime values given are only valid for the specific experiment, as the photoswitching is very sensitive to small deviations in the conditions.

\subsection{1 rsEGFP(N205S)}

For the proteins with negative photoswitching, the lifetimes of the on- and offstates were determined for a large number of irradiation intensity combinations as described in 4.2.2.1. The plots in Fig. 24 illustrate the dependence of the lifetimes on the irradiation intensities. The photoswitching was studied under three different illumination conditions:

- 488 and $401 \mathrm{~nm}$ LED illumination (Fig. 24a),

- 514 and $401 \mathrm{~nm}$ LED illumination (Fig. 24p), and

- 488 and $401 \mathrm{~nm}$ laser illumination (Fig. 24k and Fig. 24d).

According to the theoretical view of negative photoswitching, the higher the 488 $\mathrm{nm}$ intensity, the faster is the transfer of the chromophores to their off-states. As a result, $\tau_{\text {off }}$ decreases with increasing $488 \mathrm{~nm}$ intensity. Since, in this idealized view, the off-switching is not influenced by the $401 \mathrm{~nm}$ irradiation, the $\tau_{\text {off }}$ curves for all $401 \mathrm{~nm}$ intensities are similar. The transfer to the on-state, on the other hand, is mediated by irradiation at $401 \mathrm{~nm}$ and theoretically independent of $488 \mathrm{~nm}$ intensity. Therefore, $\tau_{\text {on }}$ is approximately constant at different $488 \mathrm{~nm}$ intensities. Higher $401 \mathrm{~nm}$ intensity causes faster on-switching, leading to lower $\tau_{\text {on }}$ values.

At $488 \mathrm{~nm}$ and $401 \mathrm{~nm}$ LED irradiation (Fig. 24a), $\tau_{\text {off }}$ showed the expected dependences, with values well in the regime useful for SOFI (15 to $80 \mathrm{~ms}$ ). While $\tau_{\text {on }}$ could be considered constant for the higher intensity of $401 \mathrm{~nm}(0.04 \%)$ as estimated, it decreased with increasing $488 \mathrm{~nm}$ intensity for the lower intensity of $401 \mathrm{~nm}(0.02 \%)$. The lifetimes at $514 \mathrm{~nm}$ and $401 \mathrm{~nm}$ LED irradiation (Fig. 24b) excellently fit the expectations. $\tau_{\text {off }}$ was almost identical for both $401 \mathrm{~nm}$ intensities and decreased with increasing $488 \mathrm{~nm}$ intensity, while $\tau_{\text {on }}$ was unaffected by changes 


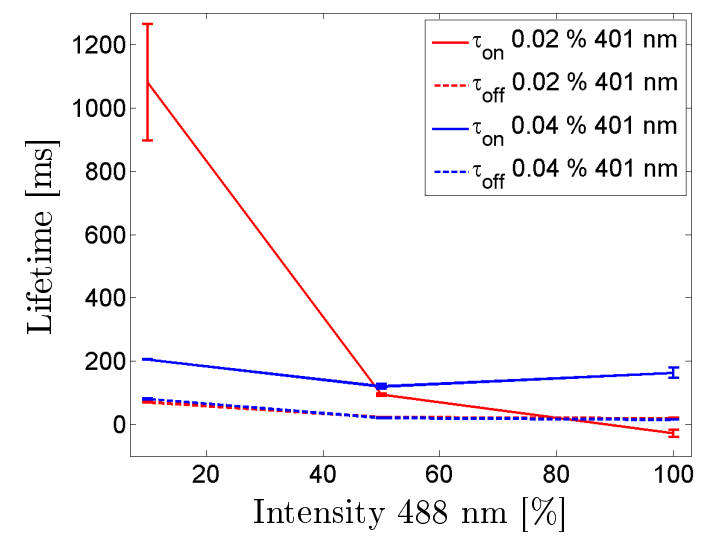

(a) 488 and $401 \mathrm{~nm}$ LED

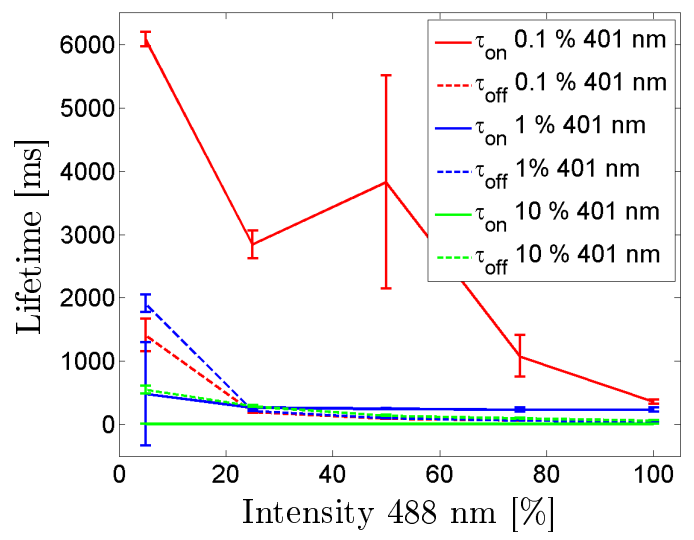

(c) 488 and $401 \mathrm{~nm}$ laser

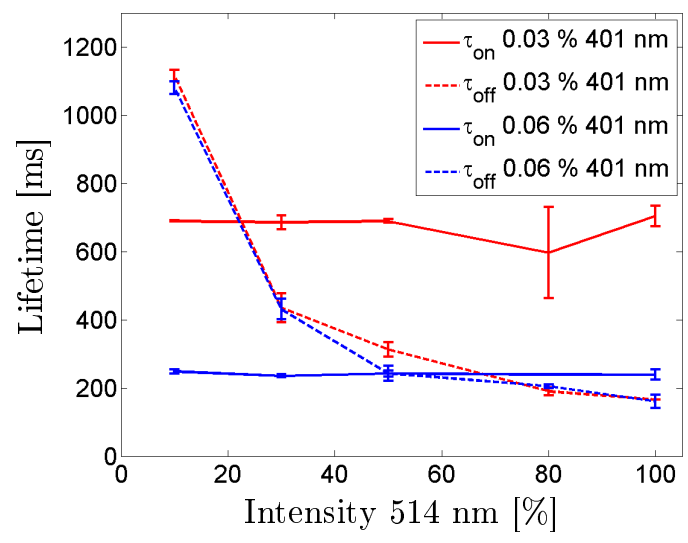

(b) 514 and $401 \mathrm{~nm}$ LED

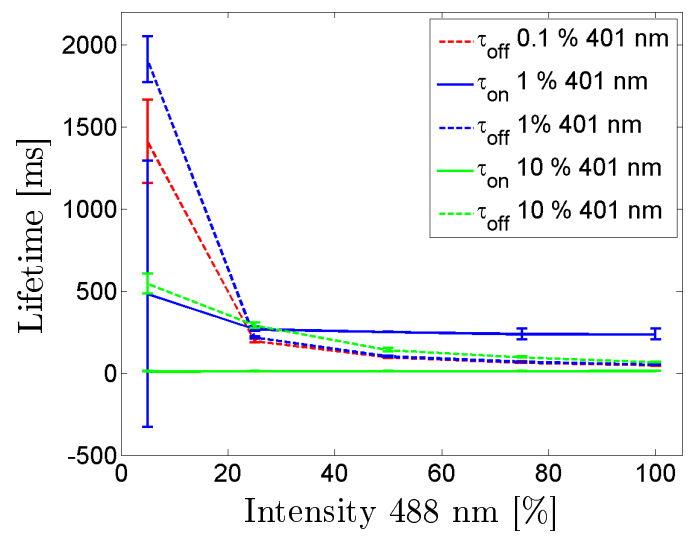

(d) 488 and $401 \mathrm{~nm}$ laser (without $\tau_{\text {on }}$ of $0.1 \% 401 \mathrm{~nm}$ )

Figure 24. rsEGFP(N205S) photoswitching under different irradiation conditions.

in $488 \mathrm{~nm}$ intensity, but depended strongly on $401 \mathrm{~nm}$ intensity. At $488 \mathrm{~nm}$ and $401 \mathrm{~nm}$ laser irradiation (Fig. 24c and Fig. 24d, rescaled for clarity), $\tau_{\text {off }}$ decreased with increasing intensity of $488 \mathrm{~nm}$ irradiation, as expected. Additionally, the $\tau_{\text {off }}$ curves for all intensities of $401 \mathrm{~nm}$ were very similar, with the exception of the values of $\tau_{\text {off }}$ for the lowest $488 \mathrm{~nm}$ intensity $(5 \%) . \tau_{\text {on }}$ was approximately constant with respect to the intensity of $488 \mathrm{~nm}$ illumination for the higher $401 \mathrm{~nm}$ intensities (1 and $10 \%)$. For the lowest intensity of $401 \mathrm{~nm}(0.1 \%)$, however, $\tau_{\text {on }}$ decreased with increasing $488 \mathrm{~nm}$ intensity.

While the photoswitching of rsEGFP(N205S) fit this model very well with only few exceptions, the behavior of all other investigated RSFPs with negative switching deviated from this view to varying degrees. 


\subsubsection{Dreiklang}

The photoswitching of Dreiklang was studied as described in 4.2.2.2. Since irradiation at $514 \mathrm{~nm}$ elicits fluorescence but has negligible influence on the photoswitching, the experiments were carried out at a constant $514 \mathrm{~nm}$ intensity. Dreiklang's onswitching depends on the intensity of the $375 \mathrm{~nm}$ irradiation and its off-switching on the intensity of $401 \mathrm{~nm}$. As for rsEGFP(N205S), Dreiklang lifetimes were plotted against the intensity of the off-switching wavelength to illustrate the dependences. $\tau_{o f f}$ is estimated to decrease with increasing intensity of $401 \mathrm{~nm}$ irradiation, due to accelerated off-switching. $\tau_{o n}$ is approximately constant with respect to the $401 \mathrm{~nm}$ intensity, but a higher intensity of $375 \mathrm{~nm}$ leads to shorter times in the off-state and therefore lower values of $\tau_{o n}$.

Figure 25 shows the photoswitching of Dreiklang at varying $401 \mathrm{~nm}$ and 375 nm laser irradiation. For clarity, three different scalings are shown: the full plot (Fig. 25a), the plot without $\tau_{\text {on }}$ for $5 \% 375 \mathrm{~nm}$ intensity (Fig. 25b), and the plot rescaled to lifetimes from 0 to $1000 \mathrm{~ms}$ (Fig. 25c). $\tau_{\text {off }}$ fit the expectations very well, as it decreased with increasing $401 \mathrm{~nm}$ intensity. Furthermore, the curves for different intensities of $375 \mathrm{~nm}$ were very similar, with the exception of the values for the lowest of the investigated $401 \mathrm{~nm}$ irradiation intensities (1\% $401 \mathrm{~nm}) . \tau_{\text {on }}$ was approximately constant with regard to the $401 \mathrm{~nm}$ intensity for the higher 375 $\mathrm{nm}$ intensities (50 and $100 \%$ ). $\tau_{\text {on }}$ for the lowest $375 \mathrm{~nm}$ intensity (5\%), however, deviated from the expectations with a very high value and standard deviation at $0.01 \%$ intensity of $488 \mathrm{~nm}$.

\subsubsection{Discussion}

In general, several additional effects can influence the photoswitching kinetics. Residual bleaching, for example, impairs the accuracy of lifetime fitting. Furthermore, thermal relaxation from the dark state leads to a fluorescence increase independent of the on-switching irradiation. This effect is especially pronounced for the long measurement times and low irradiation intensities used for the photoswitching experiments. Additionally, cross-talk can lead to switching caused by the wrong wavelength, changing the dependencies. 


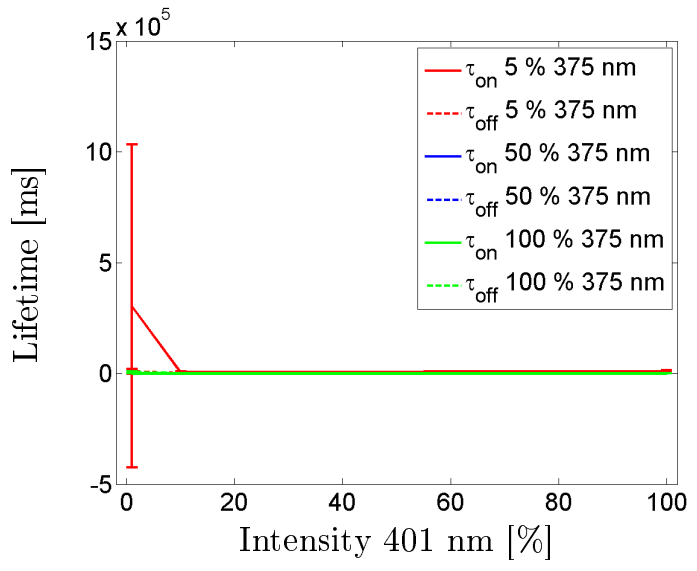

(a) 488, 401, and $375 \mathrm{~nm}$ laser

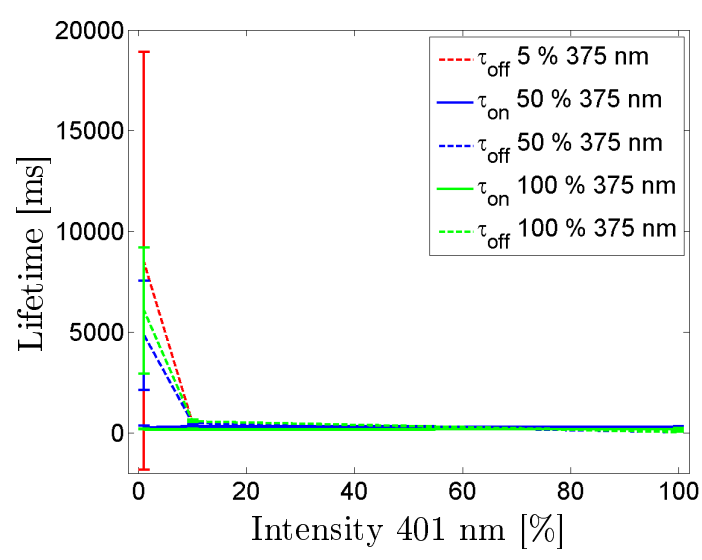

(b) 488, 401, and $375 \mathrm{~nm}$ laser (without $\tau_{\text {on }}$ of $5 \% 375 \mathrm{~nm}$ )

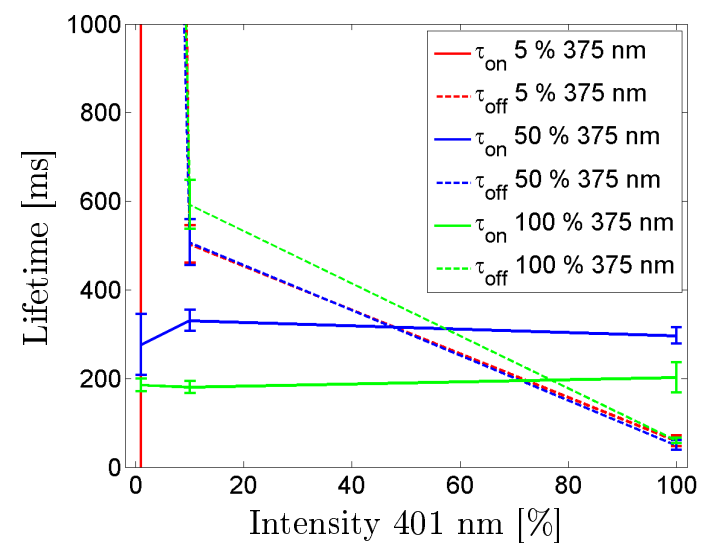

(c) 488, 401, and $375 \mathrm{~nm}$ laser

(without $\tau_{\text {on }}$ of $5 \% 375 \mathrm{~nm}$, rescaled)

Figure 25. Dreiklang photoswitching under laser irradiation.

\subsubsection{1 rsEGFP(N205S)}

The surprising decrease of $\tau_{\text {on }}$ with increasing $488 \mathrm{~nm}$ intensity at 488 and $401 \mathrm{~nm}$ LED illumination (Fig. 24a) indicates an influence of the $488 \mathrm{~nm}$ irradiation on the off-time. Although switching is most efficiently mediated by light of approximately $401 \mathrm{~nm}$, longer wavelengths can also trigger the transition from the off- to the on-state. While the emission maximum of the $488 \mathrm{~nm}$ LED used as light source was $488 \mathrm{~nm}$, shorter wavelengths were also emitted, and the shortest wavelength able to pass the emission filter was approximately $475 \mathrm{~nm}$. Consequently, a certain degree of on-switching by the $488 \mathrm{~nm}$ illumination is possible. Since the efficiency of 
the on-switching decreases with increasing wavelength, I studied whether this effect was present under 514 and $401 \mathrm{~nm}$ LED illumination (Fig. 24b). With $514 \mathrm{~nm}$ as off-switching light, the dependency of $\tau_{\text {on }}$ on its intensity disappeared, confirming a certain degree of on-switching by $488 \mathrm{~nm}$ in addition to the expected off-switching. This effect was most noticeable in the case of irradiation at low $401 \mathrm{~nm}$ intensity and consequent slow on-switching. When the intensity of $401 \mathrm{~nm}$ irradiation was high and the on-switching therefore fast, the extra transitions were not observable in the experiment, leading to an approximately constant $\tau_{\text {on }}$. As expected, fluorescence read-out and off-switching were more efficiently mediated by $488 \mathrm{~nm}$ than by 514 nm irradiation.

$\tau_{\text {on }}$ resulting from 488 and $401 \mathrm{~nm}$ laser illumination were as expected for high intensities of $401 \mathrm{~nm}$ irradiation, but deviating for the lowest intensity studied. While on-switching due to $488 \mathrm{~nm}$ irradiation is a possible reason for this behavior, it is unlikely, since in contrast to LED excitation, laser excitation only covers a spectral range of a few nanometers. Furthermore, the divergence in $\tau_{\text {off }}$ values for the lowest $401 \mathrm{~nm}$ intensity indicated thermal relaxation. Thermal relaxation causes the irradiation-independent transition of the chromophore from the off- to the on-state. The effect is most noticeable for very low irradiation intensities. This additional on-switching independent from irradiation led to a perceived reduction of the offswitching. As a result, exponential fits of the fluorescence decay yielded generally higher $\tau_{o n}$ values. The large variations in the values were probably due to fluctuations in the extend of the thermal relaxation. Likewise, additional on-switching by thermal relaxation changes the lifetime of the off-state, possibly causing the differing $\tau_{\text {off }}$ values of different on-switching wavelengths at the lowest intensity of off-switching irradiation ( $5 \%$ of $488 \mathrm{~nm}$ ). Additionally, while the $\tau_{\text {off }}$ values differ, their order is not correlated with the intensity of the $401 \mathrm{~nm}$ illumination, indicating random fluctuations of the thermal relaxation.

Of the investigated proteins with negative switching, the best reproducibility of the lifetime modification could be achieved for rsEGFP(N205S). Additional advantages of rsEGFP(N205S) are its high photon yield per switching cycle and the fact that the lifetimes of its on- and off-state are tunable to values very suitable for SOFI.

Regarding the light source, the results show clearly that photoswitching control is best exerted using laser irradiation. With laser irradiation, undesired on-switching is prevented while still irradiating at the absorption maximum of the chromophore, thus providing high off-switching and fluorescence efficiency. 


\subsubsection{Dreiklang}

Due to the decoupled switching, control of Dreiklang photoswitching is convenient and highly suitable for SOFI. The dependence of the lifetimes on the irradiation intensities fit well with the estimations. The most notable exception was $\tau_{\text {on }}$ at the lowest intensities of both on- and off-switching irradiation. As is obvious from the extreme standard deviation, the results for these parameters were very different and unreliable. A likely reason is on-switching independent from irradiation by thermal relaxation, the effects of which are typically most noticeable at low irradiation intensities. The resulting additional on-switching causes higher values of $\tau_{\text {on }}$. The large variations were due to fluctuations in the extend of the thermal relaxation. Likewise, thermal relaxation caused the differing $\tau_{\text {off }}$ values of different on-switching wavelengths at the lowest intensity of off-switching irradiation. Crosstalk, on-switching caused by $401 \mathrm{~nm}$ irradiation, is improbable given the spectral properties of Dreiklang, and has not been observed before.

The lifetimes of the states for the other investigated intensities are very suitable for SOFI. Examples are off-times of 200 and $370 \mathrm{~ms}$ for 100 and $50 \%$ intensity of $375 \mathrm{~nm}$, respectively. Irradiation with $100 \% 401 \mathrm{~nm}$ intensity yields on-times of 60 ms, which is of equally excellent use for SOFI.

\subsection{Calibration of photoswitching}

The aim of the experiments described above was to determine the illumination parameters leading to fluorescence intensity fluctuations perfectly adjusted for SOFI. I was able to gain valuable insight into the various dependencies and to identify promising intensity regions as well as the best light source for photoswitching control. However, the most important finding was that the photoswitching of the chromophores is extremely sensitive to small, unavoidable changes of conditions. These conditions include the cellular environment of the proteins, or a slightly differently adjusted beam path and the resulting variations in surface power density. Due to this sensitivity, the lifetimes frequently deviated by factor five or even ten for data sets recorded in different sessions, although great care was taken to achieve identical conditions.

Consequently, specific intensity settings were no reliable means to reproducibly induce the desired fluctuations in the chromophores. To solve this problem, I devised calibration measurements carried out directly before the actual imaging similar to 
the experiments described in 4.2.2. Based on the previous kinetics experiments, the photoswitching behavior is estimated, and the conditions most likely to yield the required lifetimes for the particular protein can be chosen. The actual switching at the chosen parameters is then screened by a calibration experiment, and the conditions can be modified accordingly, if necessary. Thus, the switching can be directly controlled and monitored.

Naturally, this calibration depends on the accuracy of the measured on- and offstate lifetimes. Any problems caused by crosstalk can be avoided by careful choice of the wavelengths. Furthermore, both crosstalk and thermal relaxation are only noticeable at very low switching intensities, and are negligible for the intensity regions suitable to induce intensity fluctuations for SOFI. Finally, SOFI is very flexible regarding the blinking behavior, and slight deviations from the assumed switching do not impair the efficiency of the technique.

\subsection{Imaging}

With the photoswitching of RSFPs under control and tunable to the requirements of SOFI, rsEGFP(N205S) and Dreiklang were chosen for the imaging of live mammalian cells. The switching of $\operatorname{rsEGFP}(\mathrm{N} 205 \mathrm{~S})$ is slower than of the other tested proteins and the number of photons emitted on average during the time of one on-state is comparatively high, ensuring a high SNR. Furthermore, the reaction of rsEGFP(N205S) to changes in irradiation intensity proved more predictable and therefore more suitable than that of any of the other evaluated FPs. The decoupled switching of Dreiklang, on the other hand, allows for the convenient adjustment of the read-out intensity separate from the intensities of the switching wavelengths.

Taking into account the necessary laser intensity to generate the corresponding switching behavior, the risk of photobleaching, and the camera capabilities on one side and the desire for overall short imaging times on the other, exposure times of 20 to $50 \mathrm{~ms}$ are ideal.

\subsubsection{Continuous excitation}

\subsubsection{1 rsEGFP(N205S)}

Combinations of on-times of 63 to $82 \mathrm{~ms}$ and off-times of 9 to $848 \mathrm{~ms}$ were evaluated for rsEGFP(N205S) imaging. While most of the tested parameter combinations 

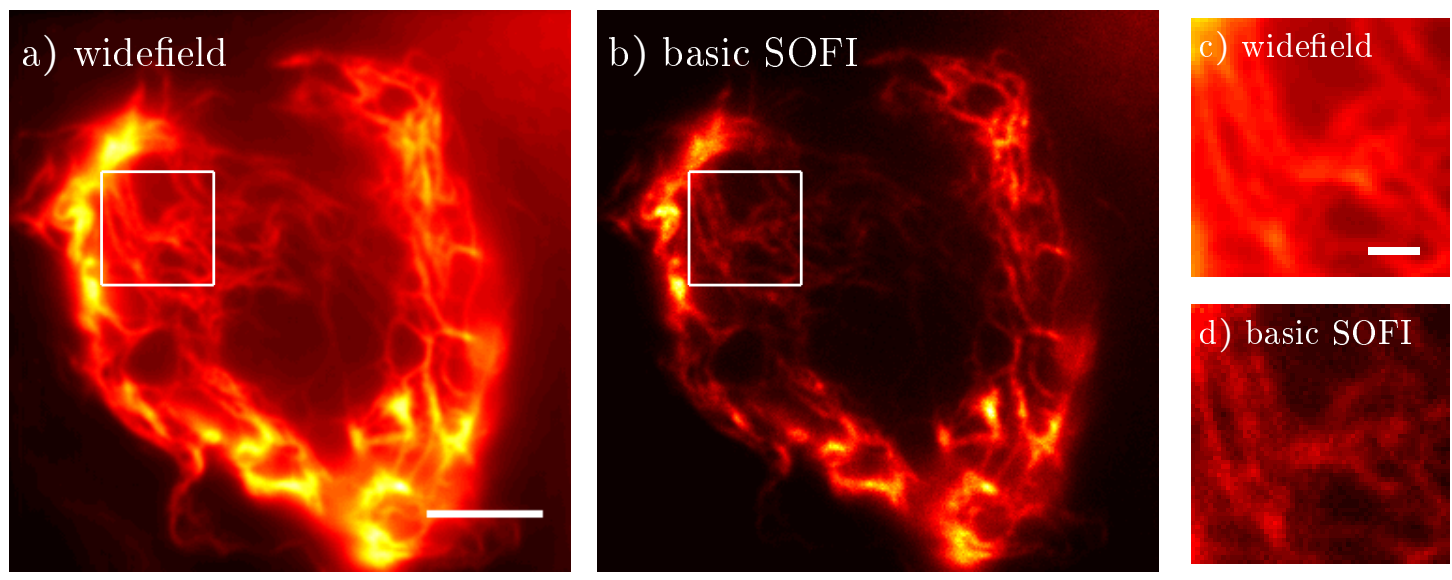

Figure 26. Widefield (a) and basic SOFI image (b) of the rsEGFP(N205S)-labeled vimentin structure of a live HeLa cell, with corresponding zoom-ins of boxed area $(c, d)$.

Exposure time: $50 \mathrm{~ms}, \tau_{o n}=9 \mathrm{~ms}, \tau_{\text {off }}=81 \mathrm{~ms}$. Scale bar: $5 \mu \mathrm{m}$, zoom-in $1 \mu \mathrm{m}$.

yielded satisfying results, best quality could be achieved with very short off-times. Shorter off-times result in a higher number of switching events per frame, leading to a more reliable detection of the switching emitter.

Images of the vimentin structure of a HeLa cell labeled with rsEGFP(N205S) are shown in Fig. 26 and Fig. 27. Since the same intensity of $488 \mathrm{~nm}$ irradiation was used for both, their on-times are near identical ( $81 \mathrm{~ms})$. The intensity of the $401 \mathrm{~nm}$ irradiation, in contrast, was double the value for Fig. 26 than for Fig. 27. resulting in a shorter off-time for the former (9 ms and $13 \mathrm{~ms}$, respectively). Compared to the widefield image, the basic SOFI images show increased resolution
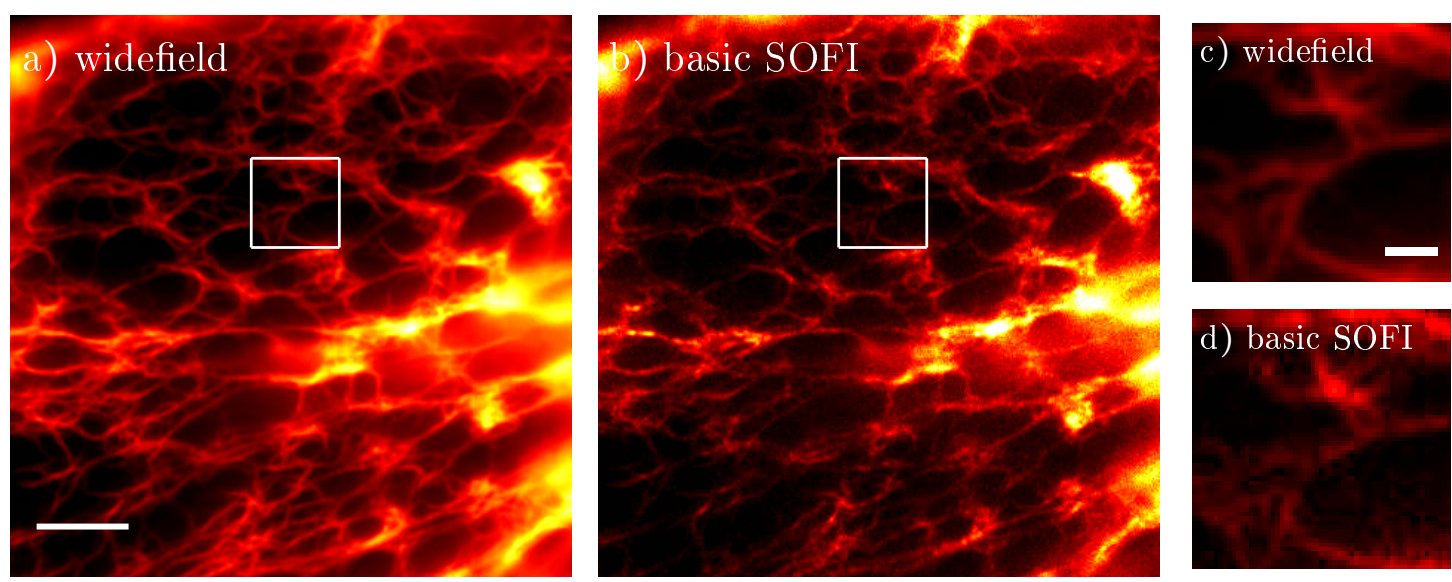

Figure 27. Widefield (a) and basic SOFI (b) image of the rsEGFP(N205S)-labeled vimentin structure of a live HeLa cell, with corresponding zoom-ins of boxed area $(c, d)$.

Exposure time: $50 \mathrm{~ms}, \tau_{\text {on }}=13 \mathrm{~ms}, \tau_{\text {off }}=82 \mathrm{~ms}$. Scale bar: $5 \mu \mathrm{m}$, zoom-in $1 \mu \mathrm{m}$. 
and reduced background.

The visibility of some of the fine, dim filaments would be improved by applying Fourier reweighting and, most importantly, linearization. The quality of Fourier reweighting and linearization depends on the accuracy of the PSF model. For QDots, for example, the PSF can be determined experimentally, by fitting Gaussian functions to images recorded from samples with single emitters. In contrast, determining the PSF for the exact conditions of RSFP SOFI data acquisition proved difficult.

\subsubsection{Dreiklang}

For Dreiklang, the on-times studied were 80 to $2746 \mathrm{~ms}$ and the off-times were 22 to $2869 \mathrm{~ms}$. As for rsEGFP(N205S), SOFI showed the best results using data with short off-times. There were, however, exceptions. For example, occasionally, a movie with both on- and off-time longer than $2000 \mathrm{~ms}$ yielded a good result. The vimentin structure of a HeLa cell labeled with Dreiklang is shown in Fig. 28. The proteins had an off-time of $200 \mathrm{~ms}$ and an on-time of $240 \mathrm{~ms}$, and were imaged at $30 \mathrm{~ms}$ exposure time. Comparing the widefield Fig. $28 \mathrm{a}$ and basic SOFI images (Fig. 28b) and the corresponding zoom-ins shows that the background is reduced and the resolution is enhanced. Linearization of the basic SOFI image based on an estimation of the PSF improves the resolution and the visibility of darker filaments
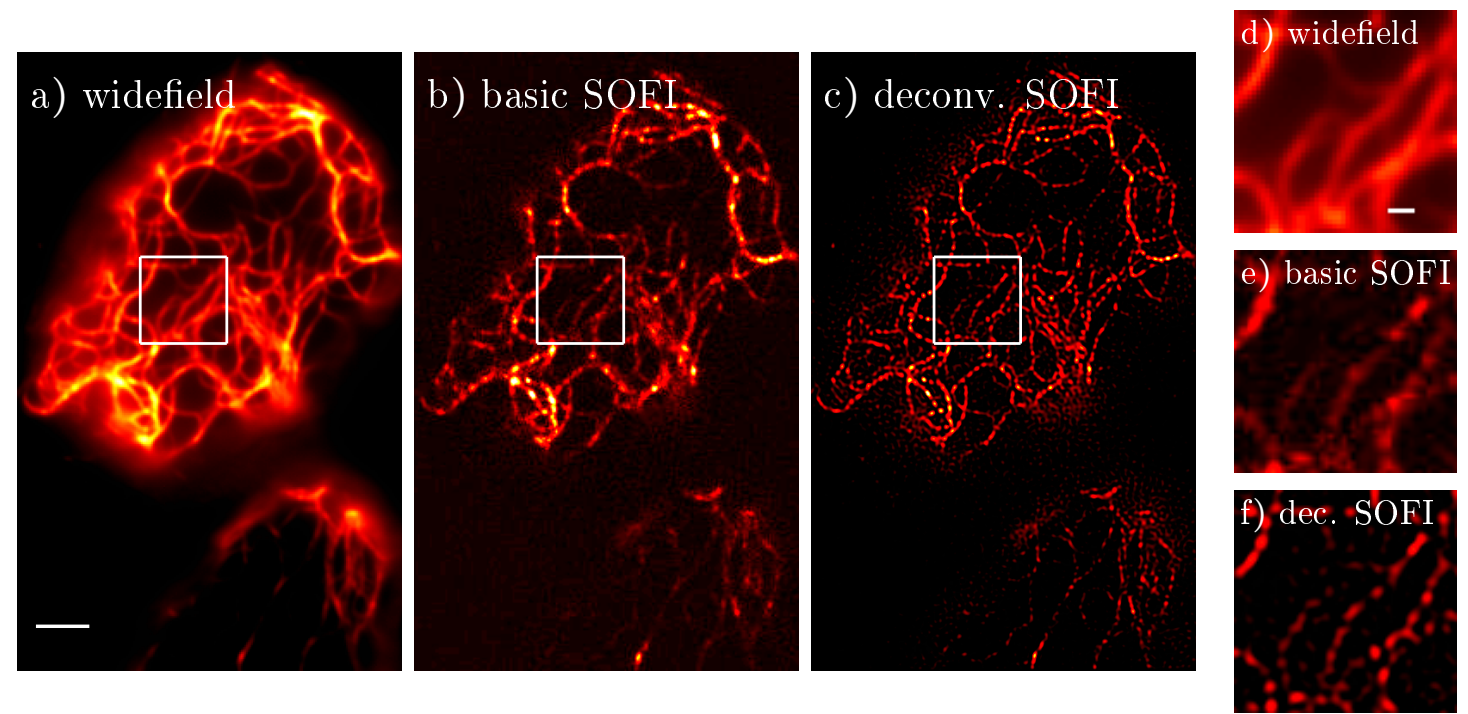

Figure 28. Widefield (a), basic SOFI (b) and deconvolved SOFI (c) image of the Dreiklang-labeled vimentin structure of a live HeLa cell, with corresponding zoom-ins of boxed area (d, e, f). Exposure time: $30 \mathrm{~ms}, \tau_{o n}=200 \mathrm{~ms}, \tau_{\text {off }}=240 \mathrm{~ms}$. Scale bar: 5 $\mu \mathrm{m}$, zoom-in $1 \mu \mathrm{m}$. 
to an even greater extent (Fig. 28k).

Structural details that are not discernible in the conventional image are made visible by the linearization of SOFI based on an estimated PSF (Fig. 29). Even the separation of two thin filaments in close proximity becomes possible, as can be seen in the zoom-ins of Fig. 29c and Fig. 29d, and the profile of the filaments along the indicated lines (Fig. 29k). The data used for the analysis of the vimentin structure was recorded with the unusual parameter combination of an off-time of $2050 \mathrm{~ms}$, an on-time of $2750 \mathrm{~ms}$, and an exposure time of $30 \mathrm{~ms}$. Surprisingly, the data yielded a basic SOFI image in a quality comparable to data with better fitting intensity fluctuations.
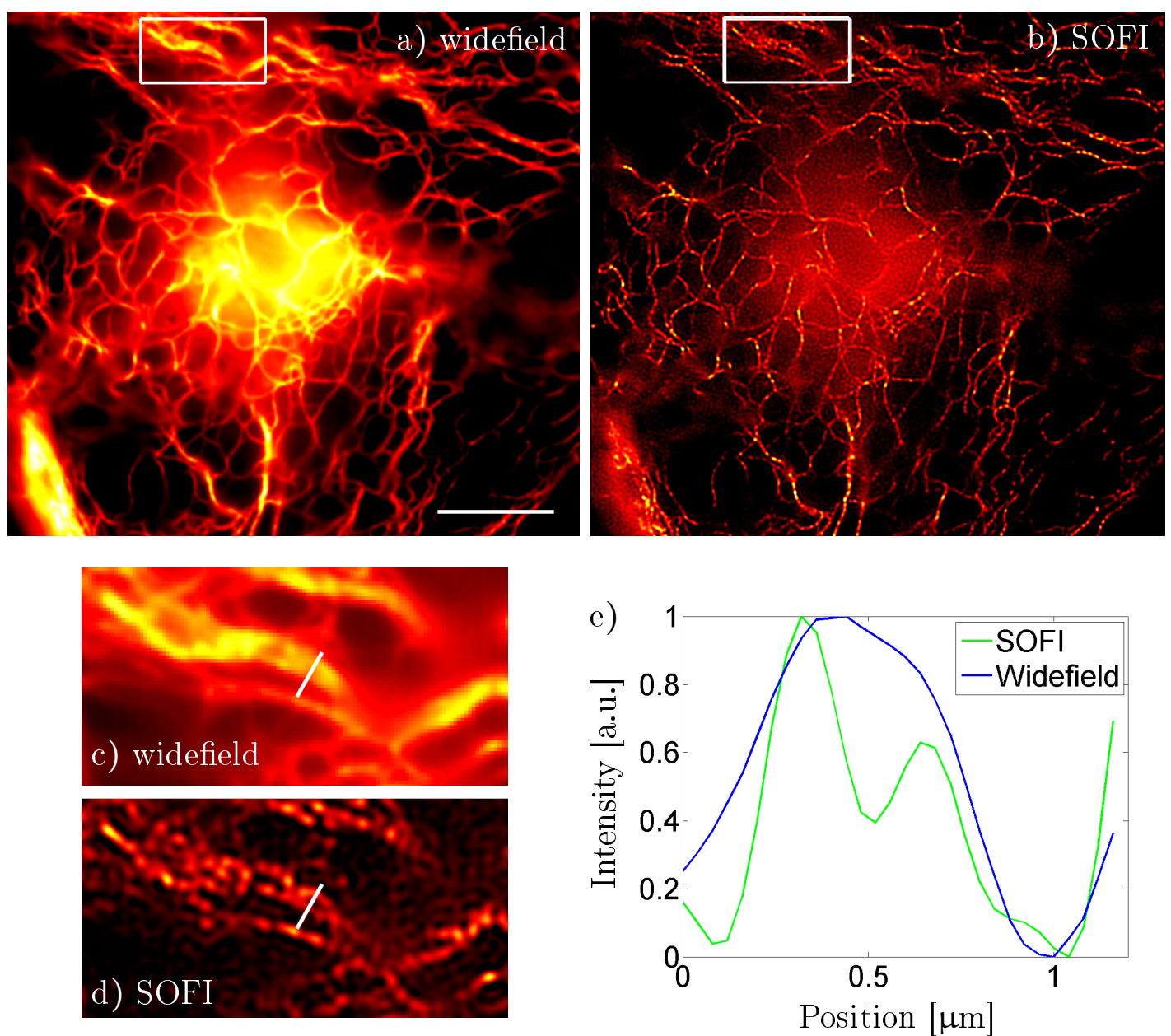

Figure 29. Widefield (a) and deconvolved SOFI (b) image of the Dreiklang-labeled vimentin structure of a live HeLa cell, with corresponding zoom-ins of boxed area $(c, d)$ and profile (e) indicated in zoom-in. Exposure time: $30 \mathrm{~ms}, \tau_{o n}=2050 \mathrm{~ms}, \tau_{o f f}=2750$ ms. Scale bar: $8 \mu \mathrm{m}$. 


\subsubsection{Pulsed excitation}

Photobleaching is detrimental to the SOFI resolution. Although rsEGFP(N205S) and Dreiklang are among the FPs most stable against photobleaching, the risk is still high, especially under continuous irradiation at all absorption maxima. Using pulsed illumination, in contrast, reduces the risk. To assess whether pulsed excitation is advantageous for SOFI, calibration measurements were performed to determine the on- and off-times at specific intensity settings and choose irradiation pulse durations. $\tau_{o f f}$ and $\tau_{o n}$ are typically used as measure for the duration of the respective state. They are defined as the time after which half of the emitters have been photoswitched under irradiation with the respective wavelength. This holds true for continuous as well as pulsed irradiation. Therefore, the lifetimes can be distributed over a desired number of frames by adjusting the pulse length. The pulse length is determined by dividing the lifetime of the state by the desired duration in number of frames.

(a) negative switching
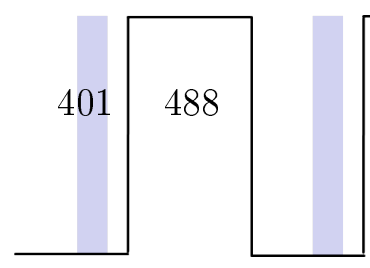
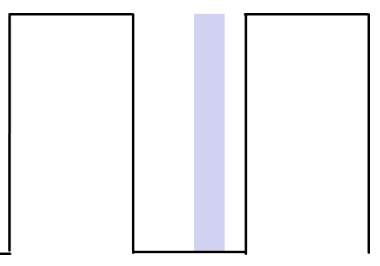

(b) decoupled switching

Figure 30. Schematic of pulsed excitation: the duration of the on- and off-states is determined by the length of the pulses. For negative switching (a), off-switching depends on the length of the $488 \mathrm{~nm}$ pulses (identical to the exposure time), while on-switching depends on the length of the $401 \mathrm{~nm}$ pulses between the frames. For decoupled switching

(b), both on- and off-times depend on the length of the respective pulses.

For proteins with negative switching, like rsEGFP(N205S), $488 \mathrm{~nm}$ irradiation is synchronized with the exposure time of the camera, thus avoiding unnecessary irradiation at times without detection. The exposure time is chosen to fit the desired ratio of on-time to frames, in the same way as for continuous irradiation. For example, if $\tau_{\text {off }}=90 \mathrm{~ms}$ and the emitters are required to be in the on-state for three frames on average, the exposure time is set to $30 \mathrm{~ms}$.

In contrast to continuous excitation, on-switching is mediated by short pulses of the on-switching wavelength $(401 \mathrm{~nm})$ between the pulses of $488 \mathrm{~nm}$ irradiation and simultaneous detection (Fig. 30a). The number of frames over which the off-state is distributed is controlled by adjusting the pulse duration. If $\tau_{o n}=10 \mathrm{~ms}$, for 
example, $401 \mathrm{~nm}$ pulses of $5 \mathrm{~ms}$ lead to off-states lasting two frames on average.

For Dreiklang imaging, illumination with the read-out wavelength $514 \mathrm{~nm}$ and camera exposure are synchronized as well. Due to the decoupling of fluorescence and photoswitching, the intensity of $514 \mathrm{~nm}$ and the exposure time can be chosen freely, while the on- and off-switching is controlled by the length of the successive pulses of $401 \mathrm{~nm}$ and $375 \mathrm{~nm}$ in between frames (Fig. 30p). Nevertheless, calibration measurements and movie acquisition for SOFI were carried out at the same intensity of $514 \mathrm{~nm}$ to avoid any possible deviations.
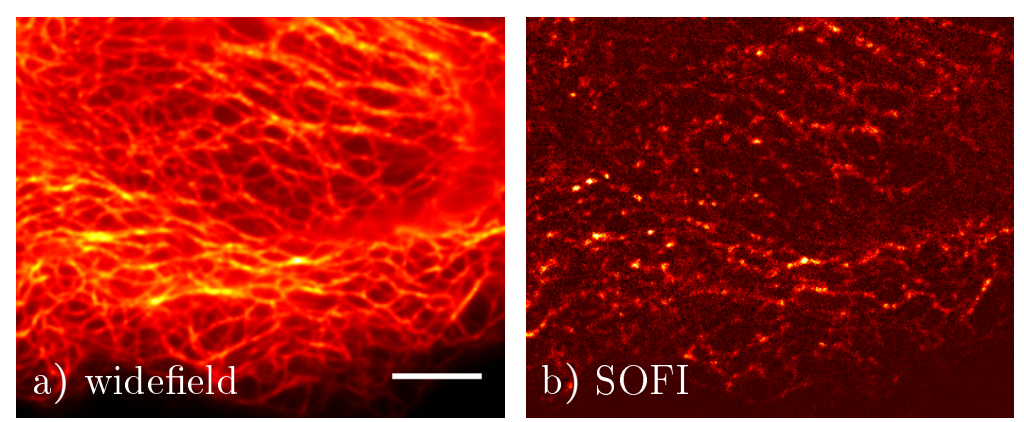

Figure 31. Widefield (a) and basic SOFI image (b) of the Dreiklang-labeled vimentin structure of a live HeLa cell with pulsed excitation. Exposure time: $50 \mathrm{~ms}, \tau_{\text {on }}=3$ frames, $\tau_{\text {off }}=5$ frames. Scale bar: $5 \mu \mathrm{m}$.

Imaging results, however, were disappointing. Image quality of many data sets recorded with different combinations of pulse duration and exposure time were compared for both Dreiklang and rsEGFP(N205S). Despite well-adjusted photoswitching and high SNR, SOFI imaging was not satisfactorily reproducible with pulsed excitation. Exemplary images of vimentin labeled with Dreiklang and imaged at pulsed excitation are shown in Fig. 31. On- and off-states were adjusted to a duration of five and three frames, respectively.

\subsubsection{Discussion}

Tunable RSFPs were determined to be excellent labels for live cell SOFI. Photoswitching control with continuous illumination at carefully chosen intensities yielded good quality images for a variety of photoswitching characteristics. Exceptions were very long off-times, which resulted in a number of switching events per emitter and movie not sufficient for SOFI computation. Linearization of the SOFI intensity response allowed for the resolution of filaments in close proximity that could not be resolved in widefield imaging. 
Interestingly, in some cases, good SOFI results could be obtained with Dreiklang despite extremely long lifetimes of both the on- and the off-state. An example was shown in Fig. 29, calculated from a data set with an exposure time of $30 \mathrm{~ms}, \tau_{\text {on }}$ $=2050 \mathrm{~ms}$, and $\tau_{\text {off }}=2750 \mathrm{~ms}$. Theoretically, at such conditions, the number of transitions from the on- to the off-state and back of each chromophore during the course of the movie is too low for SOFI. Additionally, the fluorescence of each on-state is distributed over a large number of frames, thereby lowering the SNR to an unacceptable level. As a result, these parameters are not suitable for SOFI, and typically, no images could be calculated from similar data sets. Surprisingly, Dreiklang data provided a few exceptions, while all results from rsEGFP(N205S) data were in complete accord with the expectation.

A possible reason for these exceptions lies in the determination of the lifetimes. Calibration measurements are carried out on protein-filled E.coli. Although most RSFPs show slightly different behavior in bacteria than in live mammalian cells, the deviations are typically negligible and the lifetimes determined by calibration experiments are valid for the imaging of mammalian cells. Nevertheless, it is possible that the kinetics of Dreiklang are more strongly altered by the chemical and biological environment of certain cells, potentially accelerating the transitions of the chromophores. In this case, the actual photoswitching would be faster than the switching determined in E.coli and thus be suitable for SOFI. Since the reversed case, theoretically suitable parameters not resulting in good imaging results, has not been observed, this phenomenon is of little impact for Dreiklang imaging.

Pulsed excitation was evaluated as a means to reduce the risk of photobleaching compared to continuous excitation. Since photobleaching can be detrimental for SOFI image quality, data acquisition with pulsed excitation potentially leads to a higher quality of images. Irradiation and exposure times were chosen to reproduce the photoswitching behavior previously used for successful imaging at continuous excitation. In addition to potentially reduce the risk of photobleaching, pulsed excitation offers an elegant way of photoswitching control. Once lifetimes are determined with respect to specific irradiation intensities, pulsed excitation allows for convenient tuning of exposure time and switching behavior. The duration of on- and off-states can be adjusted to any desired number of frames by simply adjusting the pulse length. Adjustment by using different irradiation intensities, in contrast, requires additional calibration measurements for each change.

Surprisingly, the image quality of continuous excitation could not be achieved. The reason for this is yet unclear. It is possible that the pulsed excitation caused 
unpredicted reactions in the chromophores, resulting in photoswitching behavior deviating from expectations. However, SOFI is very flexible regarding the blinking behavior of emitters, and only a strong deviation would lead to a noticeable decline of image quality.

The general image quality of continuous excitation was slightly below the expectations compared to SOFI with other labels. Consequently, we considered possible reasons and options to increase the image quality. One of the potentially detrimental influences on SOFI is photobleaching. Although photobleaching was low, the benefits of pulsed excitation were evaluated, but unfortunately, the results remained inconclusive. Insufficient SNR or brightness of individual emitters can impair SOFI quality as well. Despite the generally lower quantum yields of RSFPs compared to QDots and organic dyes, the SNR of the data was usually excellent. Moreover, the applicability of Dreiklang as a label for localization microscopy has been demonstrated ${ }^{52}$, indicating a perfectly sufficient individual brightness. Another possibility is that the blinking of the emitters was not perfectly adjusted to SOFI requirements. However, data acquired at a large range of combinations of lifetimes and exposure times yielded almost identical imaging results. Furthermore, SOFI is typically very flexible towards the intensity fluctuations. While ideal blinking is preferable with regard to total imaging time, deviations are compensated easily, as was demonstrated with SOFI using the inherent blinking of QDots. Although SOFI can operate at much higher labeling densities than localization methods, there is an upper limit. Typically, this limit is not reached by biological samples. Nevertheless, I evaluated the effect of reduction of the labeling density on SOFI by deliberate photobleaching of the sample before movie acquisition, with no improvement of the result.

Apparently, there are either unknown complexities to RSFPs photoswitching, or so far unknown factors determining the suitability of data for SOFI. 


\section{SOFI with organic dyes}

\section{$5.1 \quad$ Organic dyes}

Organic dyes, such as Alexa Fluor 647 (Fig. 32, are extended aromatic organic molecules capable of luminescence and frequently used in immunostaining protocols. Due to the delocalization of their $\pi$-electrons, they possess HOMO-LUMO gaps corresponding to the energy of visible light. The spectral properties of an organic dye can be altered by modification of the substituents on the aromatic system. Under constant irradiation at the absorption maximum, such a fluorophore continuously cycles between the single electronic ground and first excited state $S_{0}$ and $S_{1}$, emitting a photon with each relaxation to $S_{0}$. This property is used for fluorescence microscopy, where dyes are attached to structures of interest using immunostaining techniques. Due to intersystem crossing (ISC) to the first excited triplet state $\mathrm{T}_{1}$, the fluorescence is occasionally interrupted by dark periods.

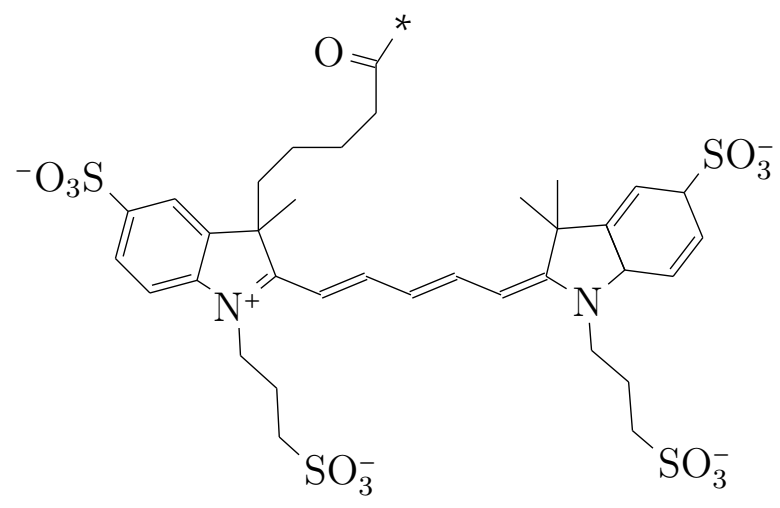

Figure 32. Molecular structure of Alexa Fluor 647

\section{2 dSTORM: (direct) stochastic optical reconstruction mi- croscopy}

All localization methods are based on generating short fluorescent states and longlived dark states in the label. This blinking allows for the subsequent recording of many images of the sample, each with a different small subset of fluorophores in the on-state. As a result, the recorded patterns of the fluorophores do not overlap in each frame. By calculating Gaussian fits of the PSF patterns, the most likely position of the emitter can be determined, which is not possible in the case of 
overlapping PSFs. The localization accuracy, and in consequence the achievable resolution, depend strongly on the number of photons emitted by each fluorophore: the more photons are detected from an emitter, the more exact is the fit and the more precise is the localization. Eventually, all individually calculated localizations are added up to one superresolved image.

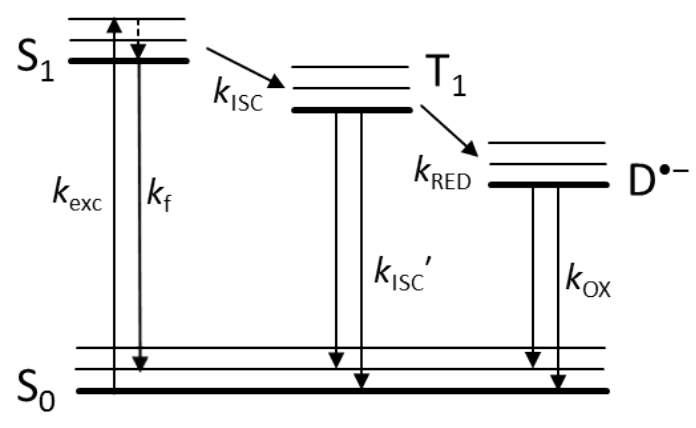

Figure 33. Energy levels of organic dye photoswitching. Fluorescence $\left(k_{f}\right)$ can be interrupted by intersystem crossing $\left(k_{\mathrm{ISC}}\right)$ to $T_{1}$, a short dark state. Form there the dye can be reduced to a long-lived dark state $\left(k_{\mathrm{RED}}\right)$ and lastly oxidized back to $S_{0}\left(k_{\mathrm{OX}}\right)$.

Organic dyes naturally provide a short off-state resulting from ISC from the first singlet state $\mathrm{S}_{1}$ to the first triplet state $\mathrm{T}_{1}$. The lifetime of the dark triplet state, however, is typically only milliseconds to seconds, too short for localization microscopy on densely labeled biological samples. To generate the required extended off-states, the triplet state is reduced. The dye is thus transfered to a non-fluorescent state with a lifetime of several seconds to tens and even hundreds of seconds, depending on the dye and the chemical environment. Figure 33 illustrates the energy levels relevant to the photoswitching. The necessary reductive chemical environment is provided by a switching buffer. The buffer typically has a $\mathrm{pH}$ in the range of 7.4 to 8.5 and contains a thiol or other reducing agent to transfer the dye from the triplet state to the reduced, non-fluorescent state. Subsequent fluorescence recovery, the transition back to the on-state at the rate $k_{o x}$, is due to reaction with oxygen.

Given sufficiently short lifetimes of the on-state, the required duration of the non-fluorescent state depends mainly on the labeling density. Regarding the onstate, a similar duration as the camera exposure is ideal. That way, all photons emitted during one on-state are recorded to one camera frame and can be used to localize the fluorophore, maximizing the accuracy. To shorten the on-state and thus the overall imaging time, the dependence of the fluorescence rate on the intensity of the exciting light is used. Increasing the excitation intensity leads to faster fluorescence cycling and, consequently, more instances of ICS per time. As a result, the 
population of the triplet state increases and the on-time shortens. In summary, the on-state is shortened by increasing the excitation intensity, thiol concentration, and $\mathrm{pH}$, while the off-time is shorter for a higher $\mathrm{O}_{2}$ concentration. Detailed parameters for induction of photoswitching in a variety of commonly used dyes can be found in the literature 62[63.

An additional way to tune the blinking, namely to shorten the off-times, is irradiation at $\sim 400 \mathrm{~nm}$, which promotes return to the on-state. Consequently, the photoswitching adjusted for STORM by way of switching buffer and excitation intensity can be easily modified to be perfectly suited for SOFI, providing a valuable addition to label control for SOFI 62[64|65.

If the conditions cannot be adjusted properly to STORM requirements, for example if the labeling density is too high or the off-states are too short, the PSFs of the individual emitters overlap in the individual frames of a movie. Localization softwares (for example rapidSTORM ${ }^{66}$ ) typically aim to reject structures consisting of more than one PSF, leading to loss of potential localizations. If by mistake the overlapping PSFs are not rejected but treated as the PSF of a single emitter instead, this results in mislocalizations. Both loss of localizations and mislocalizations severely reduce the image quality. In contrast, SOFI quality is not diminished by both high labeling density and blinking with only short off-times. SOFI is therefore a potential alternative in cases where adjustment of the photoswitching to STORM requirements is not possible.

\subsection{Organization of the axon initial segment}

The axon initial segment (AIS) is a specialized part of mammalian neurons associated with action potential generation as well as regulation of the integration of synaptic input [67. The AIS membrane displays a high density of voltage-gated sodium channels $\left(\mathrm{Na}_{\mathrm{V}}\right)$. These channels react to the input from synapses and open when the membrane potential in their vicinity reaches a certain threshold value. Opening of the channels causes an influx of sodium ions that changes the membrane potential further. Thus, an electric potential wave propagates along the axon towards the axon terminal, where it triggers the release of neurotransmitters. Despite the importance of the distribution and gating kinetics of $\mathrm{Na}_{\mathrm{V}}$ for $\mathrm{AP}$ generation and neuronal signal transduction, the density of sodium channels in the AIS membrane is still a matter of controversy 68 .

Sodium channels are localized to the AIS membrane by association with the 
structural proteins spectrin and ankyrin- $\mathrm{G}$ 69/70. Spectrin is a tetrameric cytoskeletal protein that helps to maintain the structural integrity of the plasma membrane by linking the ankyrin-G-sodium-channel-complex to the underlying actin network. The extended, flexible $(\alpha \beta)_{2}$ spectrin tetramer found in the AIS consists of two $\alpha$ and two $\beta$-spectrins forming antiparallel heterodimers 71 . The N-termini of the $\beta$ spectrin subunits are located at either end of the tetramer and both C-termini at its center. The spectrin tetramers are arranged parallel to the AIS. Their ends, with the N-termini of $\beta$-spectrin, are located along rings wrapped around the circumference of the AIS. The distance between these rings is approximately 190 to $200 \mathrm{~nm}$. Similarly, the sodium channels are located along rings wrapping around the AIS, halfway between the rings formed by the N-termini of $\beta$-spectrin. The organization of sodium channels and spectrin in the AIS is schematically illustrated in Fig. 34 . Immunostaining the $\mathrm{N}$-terminal regions of the splicing variant $\beta I V$-spectrin, which are located at the rims of the tetramer, leads to periodic rings in the fluorescence image, provided that the resolution is sufficiently high. Fluorescently labeled sodium channels appear as similar rings in the images.

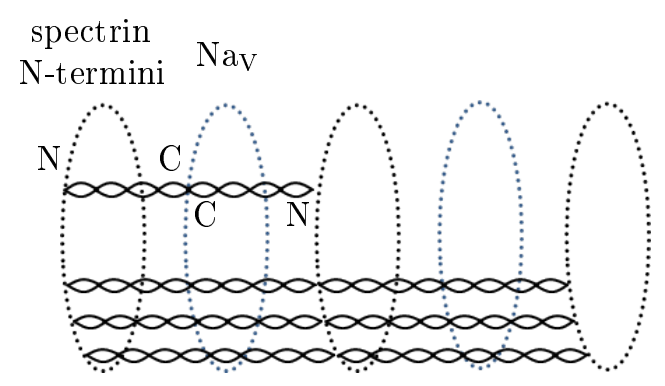

Figure 34. Schematic of the organization of sodium channels and spectrin in the axon initial segment. Spectrin tetramers are arranged along the AIS, with the N-termini of $\beta$-spectrin located along rings (only few spectrin tetramers shown for clarity). Sodium channels are located along rings halfway between the $\beta$-spectrin $N$-termini.

\subsection{Blinking flexibility}

STORM requires blinking labels with very long off-times, while on-times are ideal when they last for about one movie frame. SOFI, in contrast, yields excellent results for many different types of blinking, although total imaging time increases in the case of suboptimal blinking. SOFI therefore is a promising alternative when STORM-like blinking is not feasible.

Three different types of data sets were evaluated with both methods, among them 
data with photoswitching optimized for STORM and for SOFI. In addition, data was studied that was intended for STORM analysis, but where the aim of STORM compatible blinking could not be achieved. The structures chosen for evaluation were spectrin and sodium channels of mouse hippocampal neurons. Spectrin and sodium channels form alternating periodic rings around the AIS ${ }^{70}$, with a spacing of $\sim 190 \mathrm{~nm}$ between rings of the same type. Due to the reduced penetration depth of TIRF microscopy, not the whole height of the rings is imaged in all cases. The spectrin and sodium channel rings are interesting structures for the comparison of techniques, since their periodicity of $\sim 190 \mathrm{~nm}$ is just below the conventional resolution limit but reliably resolvable with well-adjusted STORM [70], whose typical achievable resolution for excellent data is 10 to $40 \mathrm{~nm}$.

The potential resolution of second order SOFI can be estimated using the PSF FWHM of the label and the resolution enhancement of factor 1.8, determined with single QDots. According to Eq. 2, the PSF under ideal conditions has a FWHM of $229 \mathrm{~nm}$ for Alexa Fluor $647\left(\lambda_{e m}=668 \mathrm{~nm}, \mathrm{NA}=1.49\right)$ and $178 \mathrm{~nm}$ for Alexa Fluor $488\left(\lambda_{e m}=520 \mathrm{~nm}, \mathrm{NA}=1.49\right)$. PSFs measured from the data had a FWHM of 299 $\pm 10 \mathrm{~nm}$ for Alexa Fluor 647 and of $244 \pm 11 \mathrm{~nm}$ for Alexa Fluor 488. Both values were calculated from ten randomly selected measurements. The deviations from the theoretical values can be attributed to optical imperfections and the refractive index mismatch between the system of the objective lens, the immersion oil and the cover glass with the refractive index of glass and the switching buffer with the refractive index of water. Regarding second order SOFI, the corresponding FWHM can be estimated to be $148 \mathrm{~nm}$ for Alexa Fluor 647 and $136 \mathrm{~nm}$ for Alexa Fluor 488, sufficiently small for the resolution of emitters at a distance of $190 \mathrm{~nm}$. However, a periodicity of $190 \mathrm{~nm}$ does not necessarily mean an emitter distance of $190 \mathrm{~nm}$.

\subsubsection{Spectrin with Alexa Fluor 647: photoswitching opti- mized for STORM}

Spectrin was immunostained with Alexa Fluor 647 and the photoswitching was optimized for STORM. The resulting data was analyzed by calculating the average over all frames to generate a widefield image (Fig. 35a and Fig. 35b), by performing SOFI analysis (Fig. 35. and Fig. 35d) and STORM analysis (Fig. 35e and Fig. 35f). Unsurprisingly, the widefield image had a high background and was poorly resolved. In comparison, the SOFI result was considerably clearer. The background was suppressed and individual rings were discernible. STORM, as expected, provided 

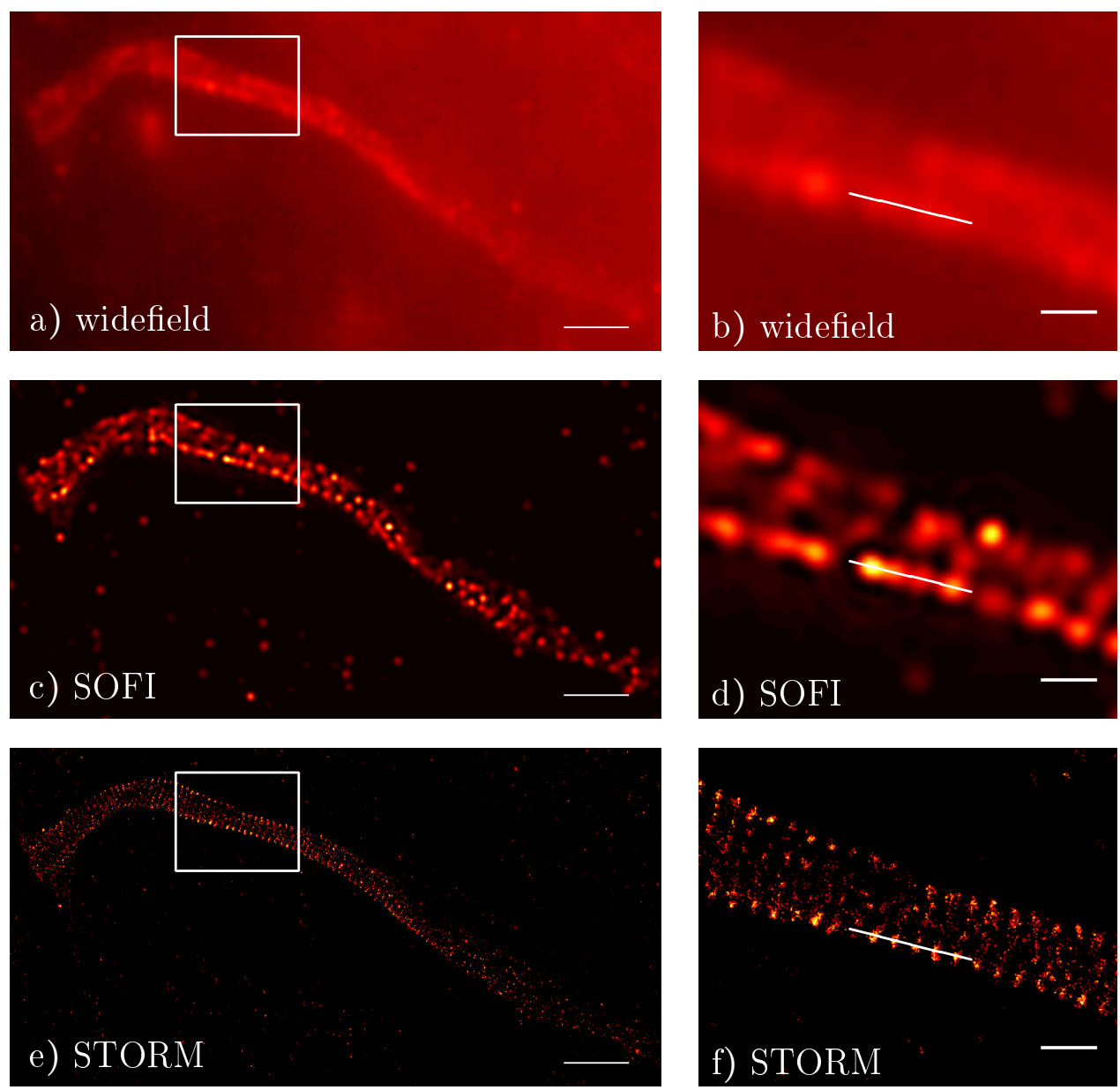

Figure 35. Widefield (a), SOFI (c), and STORM (e) image of spectrin immunostained with Alexa Fluor 647. Corresponding zoom-ins of boxed area (b, $d, f)$. Photoswitching optimized for STORM. Scale bar: $2 \mu \mathrm{m}$, zoom-in $500 \mathrm{~nm}$.

excellent resolution of the spectrin structure.

The cross section indicated in the zoom-ins along the AIS membrane (zoomins: Fig. 35. cross section: Fig. 36 was plotted after background subtraction and normalization. The cross section showed that STORM resolved periodic structures with a distance of $~ 190$ to $200 \mathrm{~nm}$, in perfect agreement with the previously reported spectrin structure 70 . In contrast, widefield imaging was not able to resolve the peaks associated with the individual rings. Regarding SOFI, the situation was ambiguous. SOFI narrowly resolved the spectrin structure in many cases, for example in the cross section plotted in Fig. 36, where four of the five rings were resolved. The peak at approximately $0.8 \mu \mathrm{m}$ was either missing or represented by the small shoulder of the neighboring peak at $1.0 \mu \mathrm{m}$. Interestingly, the positions of missing peaks in SOFI are the positions of the peaks with the weakest intensities in STORM. 


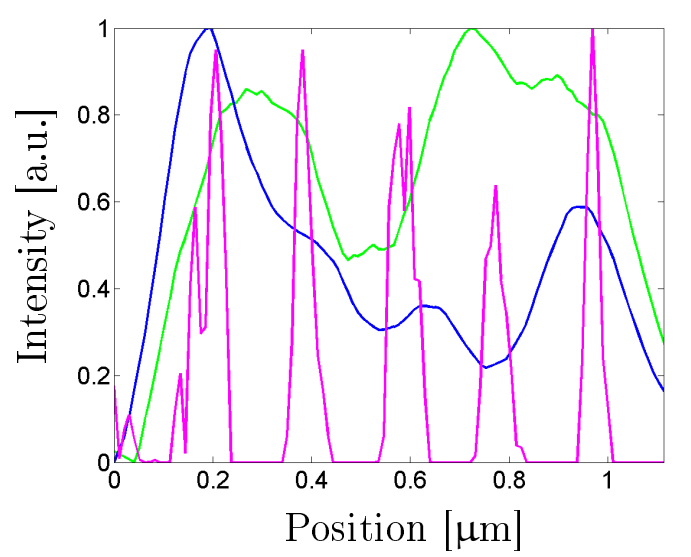

Figure 36. Spectrin immunostained with Alexa Fluor 647, STORM optimized, cross section from Fig. 35 .

The inability of SOFI to reliably resolve some of the spectrin rings can be due to the width of the rings and the length of the linker that connects the labels to the proteins of interest. The locations of the spectrin epitopes that are labeled deviate from a perfect plane. They are shifted to either side of the plane, causing a broadening of the ring and a narrowing of the space in between. Furthermore, the antibody construct that links the label to the spectrin epitopes has a length of approximately $15 \mathrm{~nm}$, broadening the imaged rings further and reducing the distance between the labels of two neighboring peaks by a maximum of $30 \mathrm{~nm}$. As a result of both these properties, the distance between the emitters labeling the spectrin rings can become so small in certain cases than SOFI is not able to resolve them.

The reason for the occasional suppression of individual peaks might be peculiarities of the photoswitching of the corresponding emitters or the labeling. For example, an unusually low number of switching events during movie acquisition due to early photobleaching can cause a very low apparent brightness in the SOFI image. A low number of fluorophores attached to the corresponding epitopes can reduce the visibility as well. Moreover, it is possible that the linearization of the emitter brightness response is not complete.

\subsubsection{Spectrin with Alexa Fluor 647: photoswitching opti- mized for SOFI}

While SOFI works well with data with STORM optimized blinking, the photoswitching can be tuned to the requirements of SOFI. Particularly, given unchanged frame 

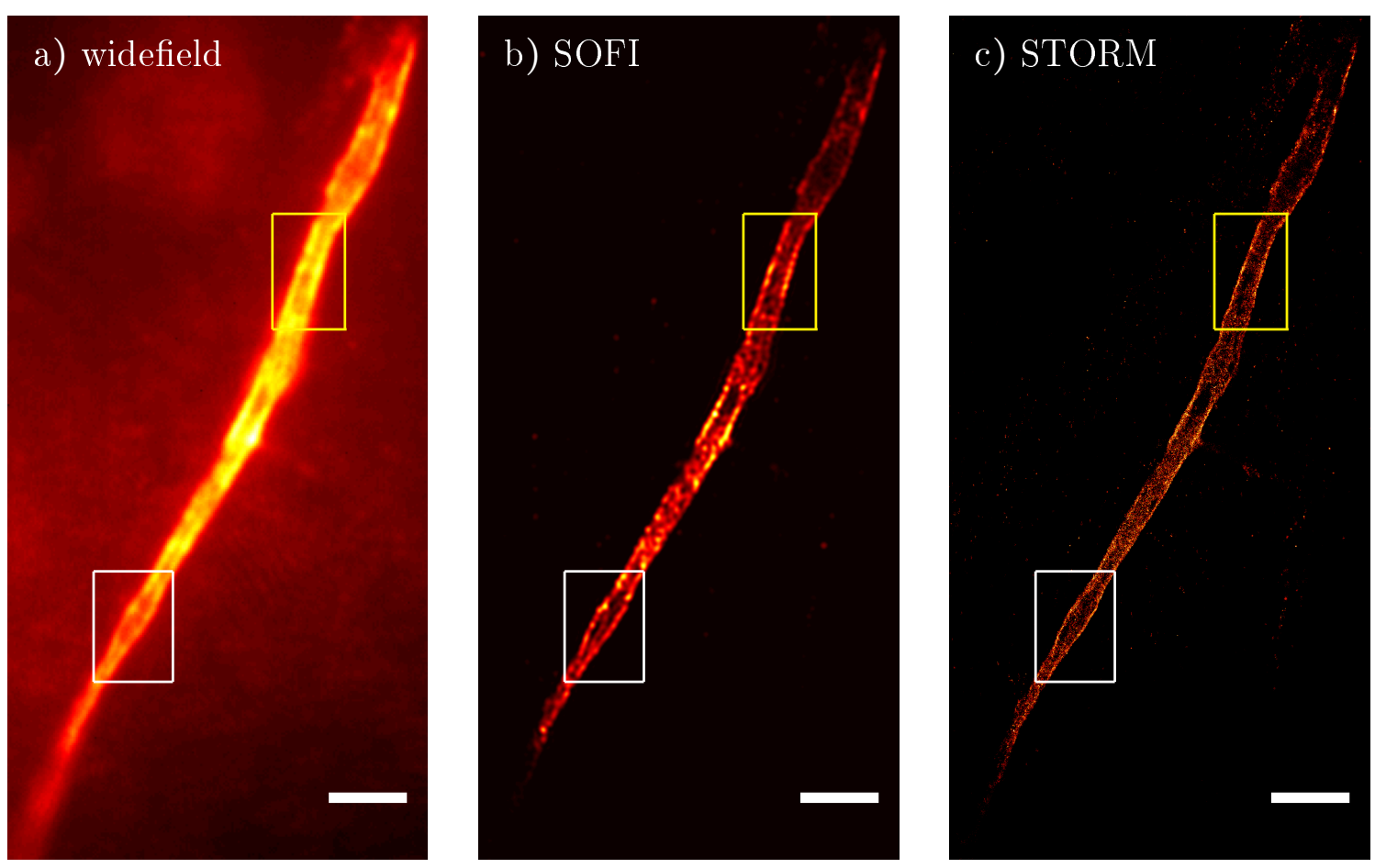

Figure 37. Widefield (a), SOFI (b), and STORM (c) image of spectrin immunostained with Alexa Fluor 647. Photoswitching optimized for SOFI. Zoom-in of boxed areas see Fig. 38. Scale bar: $3 \mu \mathrm{m}$.

rates, it is necessary to prolong the on-times and shorten the off-times compared to STORM optimized photoswitching. This is achieved by reduction of the excitation intensity at $647 \mathrm{~nm}$ and additional irradiation at $401 \mathrm{~nm}$. By tuning the irradiation intensity, the photoswitching can thus be adjusted to fit STORM or SOFI requirements as desired.

Fig. 37 shows the widefield (Fig. 37a), SOFI (Fig. 37b) and STORM (Fig. 37c) images of spectrin, calculated from data with ideal SOFI blinking. The zoom-ins of the boxed areas are shown in Fig. 38 (yellow box: a, b, c; white box: c, e, f). The widefield and SOFI results were of similar quality as previously observed for the data with STORM optimized photoswitching (compare Fig. 35). SOFI was able to resolve structures not visible in the widefield images, for which an example is given by the zoom-in of the yellow boxed area (Fig. 38d, e). The STORM analysis produced seemingly random localizations instead of resolved spectrin rings and clearly exemplified the impossibility of reliable localization if too many emitters are in the fluorescent state at a given time. Additionally, mislocalizations prevented STORM from resolving the fine structure that SOFI made visible.

To study the resolution of the spectrin ring periodicity in more detail, the cross 

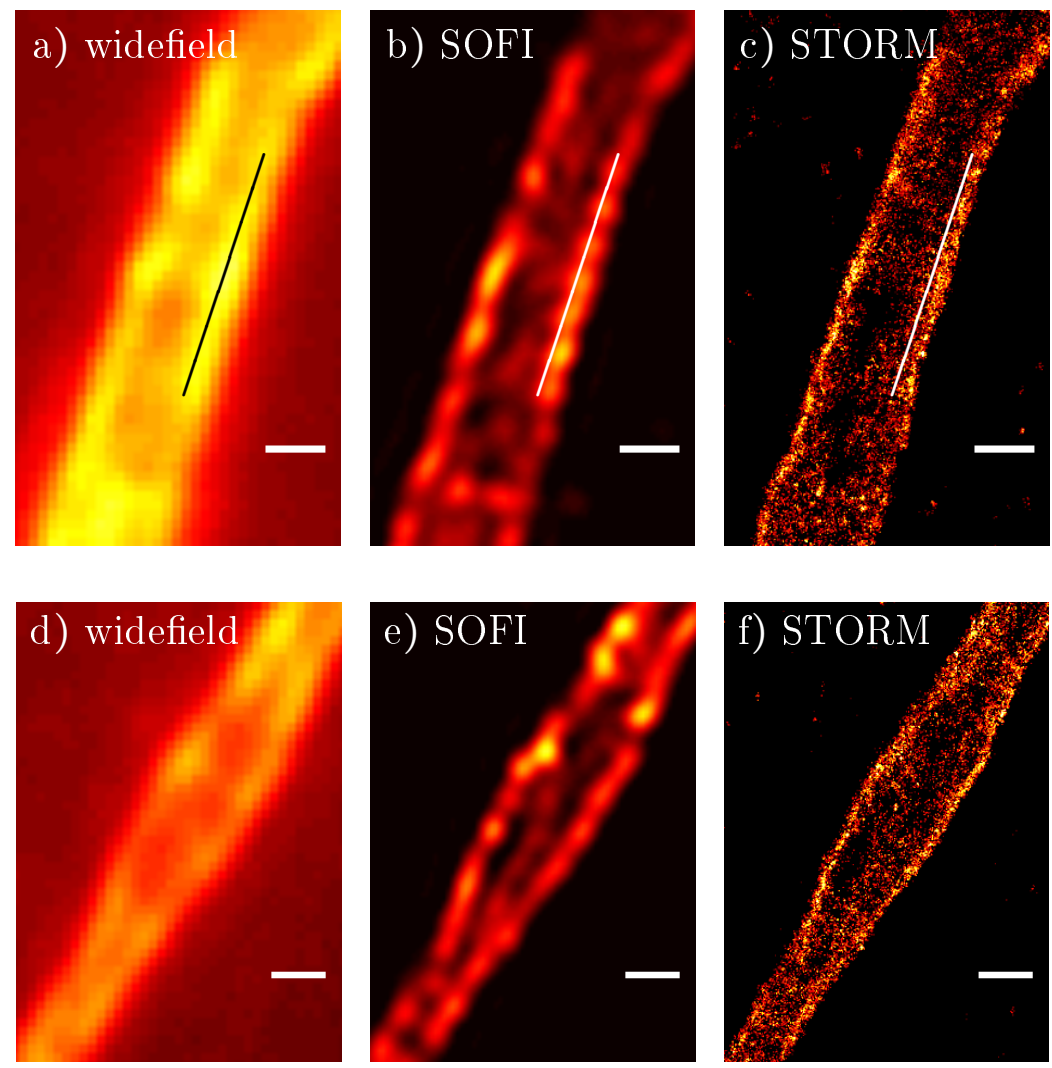

Figure 38. Zoom-ins of boxed areas in Fig. 37 (yellow box: a, b, c; white box: ds, e, f), spectrin immunostained with Alexa Fluor 647. Scale bar: $500 \mathrm{~nm}$.

section indicated in Fig. $38 \mathrm{a}, \mathrm{b}$ and $\mathrm{c}$ was plotted (Fig. 39). Provided that the resolution is sufficient, the spectrin rings are represented as periodic peaks at a distance of 190 to $200 \mathrm{~nm}$. As expected, widefield imaging was not able to resolve

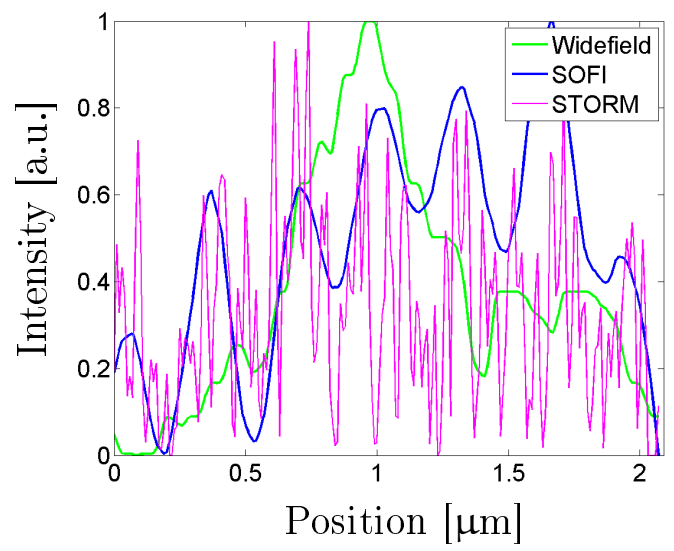

Figure 39. Spectrin immunostained with Alexa Fluor 647, SOFI optimized, cross section from Fig. 38. 
the spectrin rings. Likewise, due to the unsuitable photoswitching conditions, the intensity distribution in the plot of the STORM cross section was noisy and differed considerably from the clearly resolved peaks obtained for STORM optimized blinking. The cross section from the SOFI image, conversely, showed regular peaks at a surprising periodicity of approximately $300 \mathrm{~nm}$, while the periodicity of the spectrin rings is 190 to $200 \mathrm{~nm}$. Although some of the peaks have shoulders, merging of neighboring peaks seems unlikely to produce such a regular pattern.

\subsubsection{Sodium channels with Alexa Fluor 488}

Unlike Alexa Fluor 647, which is highly suitable as a STORM label, the photoswitching of Alexa Fluor $488 \mathrm{~nm}$ often proves more difficult to adjust. Alexa Fluor
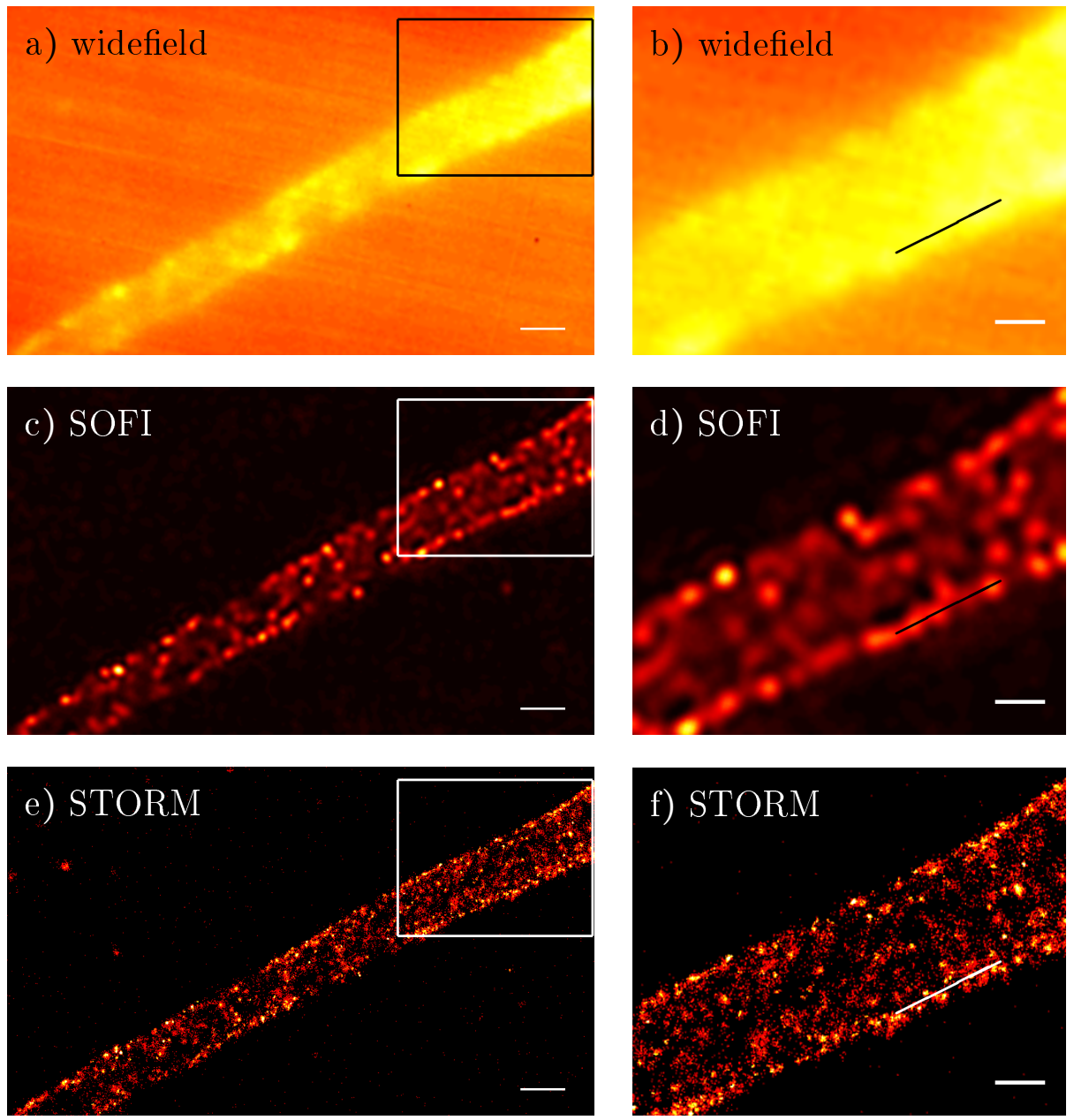

Figure 40. Widefield (a), SOFI (c), and STORM (e) image of sodium channels immunostained with Alexa Fluor 488. Corresponding zoom-ins of boxed area (b, d, f). Emitter off-states are too short for STORM analysis. Scale bar: $1 \mu \mathrm{m}$, zoom-in $500 \mathrm{~nm}$. 
488 is therefore prone to blinking incompatible with STORM, for example due to suboptimal excitation intensities or chemical environment of the emitters. If suitable conditions can not be achieved, shorter off- or longer on-states than required are the result. Such data is unsuitable for single molecule localization, but SOFI analysis is potentially advantageous.

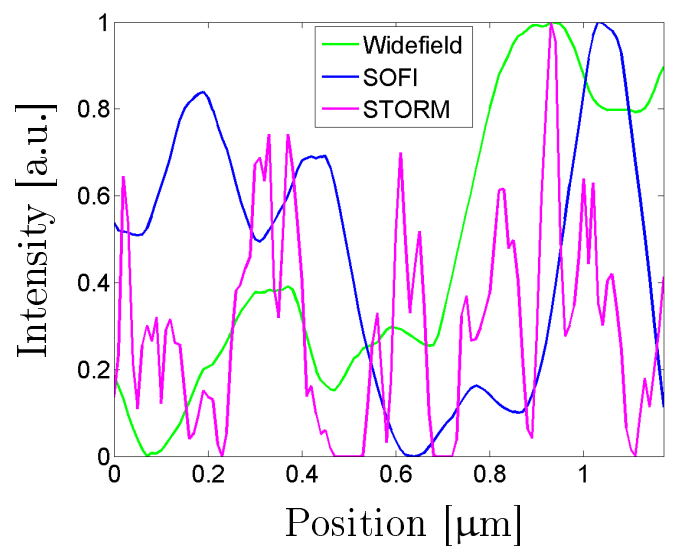

Figure 41. Sodium channels with Alexa Fluor 488, cross section from Fig. 40 .

To study the applicability of SOFI in the case of difficult photoswitching control, a movie of sodium channels labeled with Alexa Fluor 488 was analyzed (Fig. 40). The resulting widefield and SOFI images were comparable to those from SOFI and STORM optimized blinking, with poor quality of the widefield image and reduced background and increased resolution due to SOFI. In the STORM image, however, the localizations were almost uniformly distributed over the axon, and no individual structures could be distinguished. Since rings of sodium channels with a periodicity of 190 to $200 \mathrm{~nm}$ were expected, this illustrated the poor STORM suitability of the blinking. This result was similar to the result obtained from analysis of data with SOFI optimized photoswitching. The plot of the cross section indicated in the zoom-ins in Fig. 40 is shown in Fig. 41. In the cross section of the STORM image, no clear structures are recognizable and the plot is very noisy. In contrast, the SOFI cross section shows clear peaks with distances of approximately 200 and on one occasion $400 \mathrm{~nm}$. This suggests a missing peak at approximately $0.6 \mu \mathrm{m}$, potentially due to early photobleaching.

\subsubsection{Discussion}

Widefield, STORM and SOFI results were compared for three different types of data. The photoswitching of Alexa Fluor 647 bound to spectrin could be adjusted at will, 
therefore data optimized for STORM as well as for SOFI was generated using this system. Regarding sodium channels immunostained with Alexa Fluor 488, the aim was STORM compatible photoswitching. Unfortunately, in our experiments, this aim could not always be achieved due to insufficient excitation intensity and suboptimal chemical environment, resulting in unsuitably long on- and short off-states.

As expected, widefield images, generated by averaging over all frames, were similar for all data types. STORM analysis proved very useful for the data with photoswitching exactly tuned to fit STORM requirements, but was prone to mislocalizations and unable to resolve the structural details of spectrin and sodium channel distribution for all other types of data. SOFI results were similar for all types of photoswitching. Although not reaching the resolution of well-adjusted STORM, SOFI was able to increase the resolution and reduce the background in all cases.

The STORM optimized photoswitching (spectrin with Alexa Fluor 647, 5.4.1) yielded STORM images with well resolved spectrin rings. The rings were spaced at approximately $200 \mathrm{~nm}$, fitting with the previously reported $194 \pm 15 \mathrm{~nm}$ [70. The SOFI analysis of the same data showed the same periodic structure. However, not all ring parts could be resolved in all cases, or the signals of individual rings were suppressed to the extent that they were hardly detectable in images and cross sections. An example is the peak at $~ 0.8 \mu \mathrm{m}$ in the cross section plot (Fig. 36).

The SOFI optimized photoswitching (spectrin with Alexa Fluor 647, 5.4.2) was, as expected, unsuitable for single molecule localization, causing mislocalizations and rendering STORM analysis unable to resolve structural details. SOFI analysis of the same data revealed previously hidden details as well as periodic peaks of fluorescence intensity in the AIS membrane, as is anticipated for spectrin rings. Surprisingly, the distance between the evenly spaced peaks was approximately $300 \mathrm{~nm}$.

The photoswitching of Alexa Fluor 488 (sodium channels, 5.4.3) could not be adjusted for STORM. Consequently, the STORM result was similar to that of SOFI optimized photoswitching. The image was calculated to determine the suitability of SOFI analysis as an alternative approach in such cases. The SOFI image shows peaks with distances that suggest a missing peak at approximately $0.6 \mu \mathrm{m}$. An additional peak at that position leads to the expected periodicity of 190 to $200 \mathrm{~nm}$.

The FWHM of the PSFs of Alexa Fluor 647 and Alexa Fluor 488 were measured to be $299 \pm 10 \mathrm{~nm}$ and of $244 \pm 11 \mathrm{~nm}$, respectively. The measured PSFs deviate from the values calculated according to Eq. 2 due to imperfections in the optical setup and the refractive index mismatch of the system. Assuming a resolution enhancement by factor 1.8, this leads to estimated values of $148 \mathrm{~nm}$ and $136 \mathrm{~nm}$ for 
the FWHM in the SOFI images, which should be sufficient to resolve emitters 190 $\mathrm{nm}$ apart. Although the periodicity of the spectrin and sodium channel rings is 190 to $200 \mathrm{~nm}$, the rings themselves have a certain width. The binding sites for the primary antibody are not located all exactly in the same plane, which narrows the gap between the signals emitted by the fluorophores attached to the rings. Furthermore, a primary and secondary antibody together have a length of about $15 \mathrm{~nm}$, further broadening the area where the fluorophores can be located. The length of the antibody linker can reduce the distance between fluorophores attached to two rings by as much as $30 \mathrm{~nm}$. As a result, the combination of these effects can cause the distance between the fluorophores attached to the spectrin rings to be so severely reduced that it is just below the resolution capability of second order SOFI.

Suppression of individual rings could be due to a randomly small number of photoswitching events of the labels, or an untypically low number of fluorophores on the secondary antibody attached to the position, which would reduce their visibility in the SOFI image. Another possible reason is photobleaching of the fluorophores early during data acquisition. Typically, very weak or invisible SOFI signals correspond to small STORM peaks in the data for ideal STORM conditions (Fig. 36), which fits with these explanations. Although the SOFI algorithm used for the calculations in this work corrects for the intensity imbalance introduced by the analysis, the correction is incomplete. Consequently, the apparent disappearance of very dim signals is still possible. Poor resolution of neighboring peaks could result in a periodicity larger than 190 to $200 \mathrm{~nm}$, as observed in the SOFI image of the SOFI optimized spectrin imaging. Although it seems unlikely that a regular pattern with $300 \mathrm{~nm}$ periodicity could thus be generated, shoulders are discernible on some of the peaks, suggesting insufficient resolution.

In summary, STORM is reliable only when the blinking and labeling density are excellently adjusted for STORM requirements. SOFI, on the other hand, works well with all types of data, confirming predictions from simulated data 21 , and is an interesting alternative in cases where STORM compatible photoswitching is impossible. The resolution of SOFI, however, was not sufficient to reliably resolve the investigated structures. A possibility to increase the resolution is to calculate SOFI to the third order, which provides an up to three-fold resolution increase. 


\section{Conclusions and outlook}

Stochastic optical fluctuation imaging achieves superresolution by analyzing the independent temporal intensity fluctuations of labels. Additionally, SOFI reduces and often completely eliminates background fluorescence, granting optical sectioning capabilities to widefield setups. As a result, SOFI is especially advantageous when samples exhibit high background fluorescence, which interferes with many other imaging techniques. In contrast to other superresolution methods, SOFI is robust against high labeling densities. Furthermore, for many labels, excitation surface power densities of only a few $\mathrm{W} / \mathrm{cm}^{2}$ are required. This allows for prolonged imaging with reduced risk of photobleaching and light-induced damage.

For SOFI analysis, a certain number of transitions between the fluorescent and the non-fluorescent state of an emitter is necessary. Reliable detection of this number of transitions in as few frames as possible is typically achieved when the emitters are in their fluorescent state for approximately two to five frames on average, and dark for about the same number of frames. Additionally, since a short overall acquisition time is desirable, fast blinking and corresponding fast frame rates are preferred. Suboptimal blinking, however, can be easily compensated by increasing the imaging time. Therefore, SOFI can make use of a multitude of blinking kinetics and a variety of labels, such as Quantum Dots, organic dyes and reversibly switchable fluorescent proteins.

In this work, the theoretical and computational background of SOFI and additional processing steps were described. Particularly advantageous for applications is the linearization of the intensity response using a deconvolution approach. Applications of SOFI were presented and the peculiarities of different fluorophores and their respective suitability for different tasks were studied.

After determining acquisition parameters fitting the unique blinking kinetics of QDots and ensuring high setup stability, SOFI with QDots was straightforward and reliable. The individual steps of SOFI computation were illustrated using the example of individual QDots, and the total resolution improvement of second order fSOFI was determined to be over 1.8-fold. Additionally, SOFI discarded of more out-of-focus-light than confocal microscopy. Due to the high setup stability and low photobleaching, a 3D image of a neuron over a z-distance of $2.6 \mu \mathrm{m}$ could be reconstructed. $\mathrm{GABA}_{\mathrm{B}} \mathrm{R}$ subunit trafficking and assembly were studied using dualcolor, 3D SOFI. The SOFI z-sections of neurons revealed structural details as well as receptor subunit colocalization. 
$\mathrm{IP}_{3}$ and ryanodine receptors in the ER membrane of a neuron support the propagation of calcium waves by releasing $\mathrm{Ca}^{2+}$ into the cytosol. Modification of the ER organization altered the calcium waves, suggesting a dependence of the waves on the distribution of the receptors. To link the alterations of the calcium signals to the modifications of the receptor distribution, 3D, dual-color SOFI was performed. Dual-color SOFI images allowed for the study of the receptor colocalization, while additional widefield images of the ER placed the receptors in the context of the cellular system. Combined with studies on the effect of ER disruption on calcium waves, these results will elucidate the dependence of calcium signaling on ER organization and receptor distribution.

For live cell imaging, intensity fluctuations suitable for SOFI were induced in RSFPs, fluorescent proteins which can be transferred between fluorescent and dark states by suitable irradiation. The fluctuations can be tuned by adjusting the irradiation conditions. Extensive evaluation of the photoswitching of different RSFPs determined Dreiklang and rsEGFP(N205S) to be most suitable. Although promising irradiation conditions could be identified, the fluorophores proved to be extremely sensitive to environmental conditions and alterations of the irradiation. As a result, calibration measurements prior to the SOFI experiments were devised to ensure the desired intensity fluctuations. Undesired switching that altered the measured switching rate constants was observed for certain irradiation conditions, but could be prevented by the use of laser light for excitation and photoswitching. Based on the parameters identified by calibration measurements, live cell SOFI of mammalian cells was demonstrated.

In order to improve the image quality, evaluation of the blinking and the individual brightness of single molecules of Dreiklang and rsEGFP(N205S) and their influence on SOFI analysis are of interest. While linearization with an estimated PSF yielded good results for RSFPs as labels, more precise information about the PSF, simulated or obtained by measurement, is desirable to reliably perform Fourier reweighting as well as linearization. Movies consisting of 3000 frames were typically recorded for SOFI with RSFPs, but a smaller number of frames is likely to suffice for reliable SOFI calculation. Hence, identifying the minimum required frame number in relation to the blinking behavior is an interesting further project. Likewise, the overall imaging time can be reduced by accelerating the blinking and the exposure time accordingly, potentially allowing for the study of cellular dynamics using SOFI. On the other hand, higher SOFI orders provide higher resolution enhancement but require longer recordings of the blinking dynamics. With high photobleaching resis- 
tance and setup stability, the resolution can thus be increased by a factor of three to four.

Furthermore, the performance of SOFI for different types of intensity fluctuation was compared to that of the widely used localization method dSTORM. While dSTORM generally provides higher resolution than SOFI, the requirements to the blinking of the emitters are rigid. In particular, the fluorophores are required to stay in the off-state much longer than in the on-state, and the necessary duration of the off-states increases with the labeling density. Typically, for organic dyes, this type of intensity fluctuations is achieved by applying a switching buffer and high irradiation intensities, but not all sample types and dyes yield the desired result. In these cases, SOFI was shown to be a valuable alternative, providing uniformly high resolution enhancement for all types of blinking without the risk of mislocalizations. Like for SOFI with RSFPs, higher orders of SOFI with organic dyes and a resulting further resolution increase, as well as a general assessment of the required number of frames in relation to the kinetics of the intensity fluctuations, are of interest. Considering the data on AIS organization, reliably resolving the individual rings should be well possible using third order SOFI.

The resolution enhancement, optical sectioning capabilities, flexibility towards intensity fluctuations and labels and modest setup requirements make SOFI a valuable technique for a many biological applications. 


\section{Setups and protocols}

\subsection{Sample preparation and imaging conditions}

\subsubsection{GABA $_{B}$ receptor immunostaining}

QDot immunostained neurons for SOFI imaging of $\mathrm{GABA}_{\mathrm{B}}$ receptor subunits were prepared by Dr. Omar Ramírez, University of Chile, Santiago de Chile.

Primary hippocampal neurons were cultured from E18 rats ${ }^{72}$ and transfected using the $\mathrm{Ca}^{2+}$ phosphate method [73]. The plasmids used for transfections were myc$\mathrm{GABA}_{\mathrm{B}} \mathrm{R} 1$ and HA-GABA $\mathrm{B} 2$ 31]. $48 \mathrm{~h}$ post transfection, neurons were processed for immunofluorescence using the primary antibodies mouse anti-HA (MMS-101R, Covance), rabbit anti-myc (C3956, Sigma-Aldrich), and the secondary antibodies anti-mouse- $\mathrm{QD}_{525}$ and anti-rabbit-QD 605 (Invitrogen). The mounting medium was Mowiol (6 g glycerol, $2.4 \mathrm{~g}$ Mowiol 4-88, 6 mL H $\mathrm{H}_{2} \mathrm{O}, 12 \mathrm{~mL} 0.2 \mathrm{M}$ Tris $\mathrm{pH} 7.2$ buffer).

\subsection{2 $\mathrm{IP}_{3}$ and ryanodine receptor immunostaining}

QDot immunostained neurons for SOFI imaging of the $\mathrm{IP}_{3}$ and ryanodine receptor were prepared by Dr. Omar Ramírez, University of Chile, Santiago de Chile.

Hippocampal neurons were prepared from E18 Sprague Dawley rats 7274 . Neurons at 14-16 days in vitro (DIV) were transfected with KDEL-RFP and ATL2-wt or ATL2-Y444A or ATL2-L384D [75] using lipofectamine 2000 (Invitrogen). After 24 - $36 \mathrm{~h}$ of expression, neurons were fixed with $4 \%$ paraformaldehyde/sucrose solution in PBS for $10 \mathrm{~min}$ at room temperature (RT), permeabilized with $0.05 \%$ NP-40 in PBS 1x for 10 min, and blocked with $0.1 \%$ FBS in PBS 1x for 10 min. Primary antibodies (anti-IP3R1 rabbit 1:300 (Upstate, 07-514), anti-RyR2 mouse 1:50 (Thermoscientific, MA3-916), anti-HA rabbit 1:300 (Sigma, H6908)) diluted in immunofluorescence solution (IF, $0.5 \%$ BSA in PBS $1 \mathrm{x}$ ) were incubated for $1 \mathrm{~h}$ at RT, followed by 3 washes with IF solution for 5 min. Secondary antibodies conjugated with QDots (anti-mouse IgG QD 525 goat F(ab')2 1:1000 (Invitrogen, Q11041MP); anti-rabbit IgG QD 605 goat F(ab')2 1:1000 (Invitrogen, Q11402MP)) were incubated for $1 \mathrm{~h}$ at RT, and then washed 3 times for 5 min with IF solution. Coverslips were mounted using Mowiol, and sealed with nail polish. 


\subsubsection{SOFI with Quantum Dots: imaging conditions}

For the acquisition of a dual-color, 3D data set for colocalization analysis, after a suitable sample position was found, z-drive and setup were allowed to settle for 20 min for a sample newly put to the sample stage, or 5 min for further measurements. After the setup had settled, any necessary refocusing was done using only the piezoz-scanner. Typically, movies for a SOFI image consisted of 3000 frames, recorded at $50 \mathrm{~ms}$ exposure time, and at an effective pixel size of $100 \mathrm{~nm}$. Fluorescence was elicited at $401 \mathrm{~nm}$ with an intensity of approximately $20 \mathrm{~W} / \mathrm{cm}^{2}$. Color channels were recorded successively on each z-position to minimize displacement between both channels at the same z-position.

\subsubsection{Spectrin and $\mathrm{Na}_{\mathrm{V}}$ immunostaining}

The neurons stained with organic dyes for SOFI imaging of the axon initial segment were prepared by Melanie Dannemeyer from the University of Göttingen, and Elinor Lazarov, Hebrew University of Jerusalem, Israel and Max Planck Institute for Dynamics and Self-Organization, Göttingen, Germany.

Primary hippocampal cultures were prepared from mice embryos (strain C57$\mathrm{BL} / 6 \mathrm{~N}, \mathrm{E} 18)$ as in $76 / 77$. For photoswitching experiments, cells were seeded in 0.1 $\mathrm{mg} / \mathrm{mL}$ polylysine-coated (Sigma-Aldrich, P2636) 4-well chambers (Thermo Scientific nunc, 155383); 60,000 to 80,000 cells per well. Cells were cultivated at $37^{\circ} \mathrm{C}$ with $8.0 \% \mathrm{CO}_{2}$. Half of the medium was exchanged for freshly prepared medium once a week. Cells were fixed at DIV22 with microscopy formaldehyde solution $4 \%$ (Merck, 100496) at $4^{\circ} \mathrm{C}$ for 8 min and permeabilized in $0.5 \%(\mathrm{v} / \mathrm{v})$ Triton-X-100 (Sigma-Aldrich, X100) at room temperature for $5 \mathrm{~min}$, followed by $1.5 \mathrm{~h}$ blocking with blocking buffer (3\% (w/v) BSA (Sigma-Aldrich, A3059) in 1x PBS and 0.1 $\%(\mathrm{v} / \mathrm{v})$ Tween20 (Sigma-Aldrich, P7949). The samples were incubated with primary antibodies anti- $\beta I V$-spectrin $(2.0 \mu \mathrm{g} / \mathrm{mL}$, Origene, TA317365) or anti-panNav $\left(1.1 \mu \mathrm{g} / \mathrm{mL}\right.$, Sigma-Aldrich, S8809-1 MG) in blocking buffer at $4{ }^{\circ} \mathrm{C}$ overnight, followed by the secondary antibody $(2.0 \mu \mathrm{g} / \mathrm{mL}$, Life Technologies, Alexa Fluor 647 anti-goat donkey, A21447 or $1.5 \mu \mathrm{g} / \mathrm{mL}$, Alexa Fluor 488, anti-rabbit goat, Jackson Immunoresearch 111-545-003), in blocking buffer at RT for 45 min. Samples were postfixed with Microscopy Formaldehyde solution $4 \%$ at RT for $5 \mathrm{~min}$.

For triple-color staining, samples were incubated with a mixture of the primary antibodies anti-MAP2 $(9.5 \mu \mathrm{g} / \mathrm{mL}$, abcam, ab5392) and anti-panNav $(1.1 \mu \mathrm{g} / \mathrm{mL}$, 
Sigma-Aldrich, S8809-1 MG) in blocking buffer at $4{ }^{\circ} \mathrm{C}$ overnight, followed by the secondary antibodies $(2.0 \mu \mathrm{g} / \mathrm{mL}$, Alexa Fluor 488, anti-mouse goat, Molecular Probes, A11029 and $2.0 \mu \mathrm{g} / \mathrm{mL}$, Alexa Fluor 647 anti-chicken goat, A21449), in blocking buffer at room temperature for $45 \mathrm{~min}$. Coverslips were mounted with ProLong Gold antifade reagent with DAPI (Molecular Probes, P36935) to glass slides.

\subsubsection{Switching buffer}

The switching buffer for modification of the photoswitching behavior of organic dyes consisted of PBS ( $\mathrm{pH} 8.5)$ containing oxygen scavenger $(4.0 \mathrm{~g} / \mathrm{mL}$ glucose oxidase (Sigma-Aldrich, G0543), $0.57 \mathrm{mg} / \mathrm{mL}$ catalase (Sigma-Aldrich, C40-100MG), 10 wt\% glucose (Sigma-Aldrich, 49158-1KG-F) and $100 \mathrm{mM} \beta$-mercaptoethylamine (Sigma-Aldrich, M6500). Chamber wells were completely filled with buffer and sealed with a regular coverslip.

\subsubsection{SOFI with organic dyes: imaging conditions}

Assistance with STORM data acquisition by Melanie Dannemeyer, University of Göttingen, is gratefully acknowledged.

Maximum laser surface power densities were $8,1.9$ and $1.6 \mathrm{~kW} / \mathrm{cm}^{2}$, for 647,488 and $401 \mathrm{~nm}$, respectively. Alexa Fluor 647 photoswitching for STORM imaging of Spectrin was achieved with $8 \mathrm{~kW} / \mathrm{cm}^{2} 647 \mathrm{~nm}$ irradiation intensity at a frame time of $9 \mathrm{~ms}$. To generate optimal SOFI photoswitching, 1 - $4 \mathrm{~kW} / \mathrm{cm}^{2} 647 \mathrm{~nm}$ power, 0.16 to $1.6 \mathrm{~kW} / \mathrm{cm}^{2} 401 \mathrm{~nm}$ power and a frame time of 20 to $30 \mathrm{~ms}$ were used. Alexa Fluor 488 photoswitching was induced with $1.9 \mathrm{~kW} / \mathrm{cm}^{2}$ power, typical frame time was $25 \mathrm{~ms}$. Typical movies consisted of 5000 frames, and the effective pixel size was $80 \mathrm{~nm}$.

\subsubsection{Reversibly photoswitchable fluorescent proteins}

RSFP samples for photoswitching measurements and live cell SOFI imaging were prepared by Dr. Nickels Jensen, group of Prof. Dr. Stefan Jakobs, Max Planck Institute for Biophysical Chemistry, Göttingen. 


\subsubsection{E.coli}

rsEGFP1, rsEGFP2 rsEGFP (N205S), rsFastLime, Dreiklang and Dronpa (M159T) were expressed from the high-copy expression vector pQE31 (Qiagen) and expressed in E.coli (strain SURE or BL21RIL) or the expression vector pBad-HisB and expressed in E.coli (strain Top10). E.coli cells were transformed with respective plasmids and grown on LB agar plates containing ampicillin over night at $37^{\circ} \mathrm{C}$. A single colony was picked and transferred to $5 \mathrm{ml}$ LB-Amp medium. Cells were grown over night at $37^{\circ} \mathrm{C}$ on a shaker. Then, the cell suspension was again diluted and the cells were grown to an OD600 of $\sim 0.5$ at $37^{\circ} \mathrm{C}$. Protein expression was induced by adding $100 \mu \mathrm{M}$ Isopropyl- $\beta$-D-thiogalactopyranosid or $0.02 \%$ Arabinose. After induction, the cells were grown at $37^{\circ} \mathrm{C}$ for one day.

Samples were prepared as described previously ${ }^{[78}$. In brief, powdered agar was washed twice in water and taken up to $2 \% \mathrm{w} / \mathrm{v}$ in water. The suspension was heated to dissolve the agar. Approximately $1 \mathrm{ml}$ of the agar solution was spread uniformly on a clean microscope slide and dried over night. $10 \mu \mathrm{l}$ of an E.coli culture was placed onto one coated slide, covered with a cover slip and sealed with silicone-based glue (Picodent twinsil, Picodent, Wipperfürth, Germany).

\subsubsection{Mammalian cells}

To create fusion constructs of Dreiklang and rsEGFP (N205S) with vimentin, Dreiklang and rsEGFP (N205S) were amplified using the primers 5'-GATCCACCGGTCGCGGCGTGAGCAAGGGCGAGGAGCTG-3' and 5'-ACAACTTAAGAACAACAATTGTTACTTGTACAGCTCGTCCATGCC-3'. The PCR fragments were cloned into the gateway destination vector pMD-tdEosFP-N39 using the restriction sites AgeI and AflII, thereby replacing the tdEosFP coding sequence against the Dreiklang or rsEGFP (N205S) sequence. The final plasmids pMD-Vim-Dreiklang and pMD-Vim-rsEGFP (N205S) were constructed by gateway vector conversion (Invitrogen) using the donor vector pDONR223-Vim.

For imaging of mammalian cells, HeLa were transfected with the respective plasmids using Turbofect (Thermo Scientific) according to the manufacturer's instructions. The cells were propagated in DMEM medium with GlutaMAX and $4.5 \mathrm{~g} / \mathrm{l}$ glucose, $10 \%$ (vol/vol) FCS, $1 \mathrm{mM}$ sodium pyruvate, $50 \mathrm{mg} / \mathrm{ml}$ penicillin and 50 $\mathrm{mg} / \mathrm{ml}$ streptomycin. Cells were grown on coverslips in Petri dishes at $37^{\circ} \mathrm{C}$ under $90 \%$ humidity and $5 \% \mathrm{CO}_{2}$. 


\subsubsection{SOFI with RSFPs: imaging conditions}

SOFI imaging of live cell samples labeled with RSFPs was conducted at $80 \mathrm{~nm}$ effective pixel size and exposure times of 20 to $30 \mathrm{~ms}$. Typical raw movies consisted of 3000 frames. Surface power densities were approximately $1.6-3.7 \mathrm{~W} / \mathrm{cm}^{2}(488$ $\mathrm{nm})$ and $0.3-0.7 \mathrm{~W} / \mathrm{cm}^{2}(401 \mathrm{~nm})$ for imaging of rsEGFP58, and approximately $2.5 \mathrm{~W} / \mathrm{cm}^{2}(514 \mathrm{~nm}), 0.06-1.6 \mathrm{~W} / \mathrm{cm}^{2}(401 \mathrm{~nm})$, and $0.01-0.1 \mathrm{~W} / \mathrm{cm}^{2}(375 \mathrm{~nm})$ for Dreiklang imaging.

\subsection{Setups}

\subsubsection{Widefield setup equipped with LEDs}

QDot SOFI imaging was carried out on an inverted widefield microscope (Olympus IX-71) equipped with an oil-immersion objective lens (UPlanSApo, 100x magnification, NA 1.4, Olympus). The fluorescence light (Spectra X light engine ${ }^{\circledR}$, Lumencor) passed an additional lens (1.6x magnification), was separated from the LED light used for excitation (emission maximum at $390 \mathrm{~nm}$; Spectra X light engine, Lumencor) with a dichroic beam splitter (FF444/520/590, Semrock) and additional excitation filters (FF01-520/35, Semrock for QD 525 and BLP02-561R, Semrock for $\mathrm{QD}_{605}$ ) before being imaged on an electron multiplying CCD camera (EMCCD; DU897-CS0-BV, Andor) with an effective pixel size of $100 \mathrm{~nm}$. 3D scanning was done using a PIFOC ${ }^{\circledR}$ P725-2DC with E709-CRD controller from Physik Instrumente (PI) $\mathrm{GmbH} \& \mathrm{Co}$. KG.

For RSFP kinetic measurements and imaging, an additional band pass filter (BrightLine HC 485/20, F39-485, Semrock) was installed to narrow the $488 \mathrm{~nm}$ emission. The $401 \mathrm{~nm}$ emission intensity was regulated using grey filters. Dichroic mirrors used were HC BS 506 (F38-506, Semrock) for the combination of 401 and $488 \mathrm{~nm}$ and $89016 \mathrm{bs}$ (Chroma) for the combination of 401 and $514 \mathrm{~nm}$.

\subsubsection{Setup for total internal fluorescence (TIRF), widefield, and confocal spinning disk imaging}

This versatile setup can be switched between different modes by altering the beam path with mirrors.

Organic dye images were recorded on an inverted TIRF microscope (Olympus 
IX-71) equipped with an oil-immersion objective lens (UApo N, 100x magnification, NA 1.49, TIRF, Olympus), a 647 nm laser (PhoxX ${ }^{\circledR} 647,140 \mathrm{~mW}$, Omicron Laserage) and a $401 \mathrm{~nm}$ laser (Cube ${ }^{\mathrm{TM}} 401,100 \mathrm{~mW}$, Coherent) in highly inclined and laminated optical sheet (HILO) configuration. A quad-edge dichroic beam splitter (Di01-R405/488/561/635, Semrock) and a quad-band excitation filter (FF01$446 / 523 / 600 / 677$, Semrock) were used to remove the laser light before imaging the fluorescence on an electron multiplying CCD camera (EMCCD; DU-885-CS0-\#VP, Andor) with an effective pixel size of $80 \mathrm{~nm}$.

For RSFP kinetic measurements and imaging, the setup was used in widefield mode with the objective lens UPlanSApo, 100x magnification, NA 1.4, Olympus. Additional lasers used were $488 \mathrm{~nm}$ (PhoxX ${ }^{\circledR} 488,60 \mathrm{~mW}$, Omicron Laserage), 514 nm (Sapphire $514 \mathrm{~nm}, 50 \mathrm{~mW}$, Coherent) pulsed by an AOTF (AOTFnC-400.650TN, AA Optoelectronic) and $375 \mathrm{~nm}$ (PhoxX ${ }^{\circledR} 375,20 \mathrm{~mW}$, Omicron Laserage). The same combination of dicroic beam splitter, excitation filter, and camera as for organic dye imaging was used for all experiments with proteins with negative switching. For Dreiklang, the beam splitter FF 444/521/608-Di01 and the excitation filter FF01 464/541/639 (both Semrock) were used.

For confocal spinning disk imaging, excitation light (Phox ${ }^{\circledR} 488,60 \mathrm{~mW}$, Omicron Laserage) was coupled to the spinning disk unit (Yokogawa CSU-X1 (M1N-E), $1500 \mathrm{rpm}$ ) via optical fibre. The objective lens used was UPlanSApo, 100x magnification, NA 1.4, Olympus. The emission was separated by filters (FF01-520/35, and BLP02-561R, Semrock) and recorded on an electron multiplying CCD camera (EMCCD; DU-885-CS0-\#VP, Andor). For 3D scanning, a PIFOC ${ }^{\circledR}$ P725-2DC with E709-CRD controller from Physik Instrumente (PI) GmbH \& Co. KG was used.

\subsubsection{Laser pulse generation}

The pulse generation was implemented by Dr. Olaf Schulz, at the time University of Göttingen.

Laser pulses for read-out and protein switching were created using an FPGA. The FIRE output of the Andor camera was fed into the FPGA to ensure synchronization of the camera with the lasers. A logical high signal of the FIRE output corresponds to exposure of the camera chip and is directly translated into a trigger pulse for the read-out laser. During the logical low signal, two pulses of different wavelengths are generated with microsecond accuracy (response time of the laser diodes or AOTF). 
The duration of each switching pulse and the time between them can be set using a LabView program.

\subsubsection{Surface power densities}

Table 3 links total laser and LED power used for RSFP photoswitching measurements to approximate surface power densities.

\begin{tabular}{|c|c|c|c|}
\hline Light source & switching & readout at & {$\left[\mathrm{W} / \mathrm{cm}^{2}\right]$} \\
\hline cyan LED & \multirow[t]{2}{*}{ negative } & \multirow[t]{2}{*}{488 nm, LED } & $\sim 17.4$ \\
\hline violet LED & & & $\sim 20.5$ \\
\hline teal LED & \multirow[t]{2}{*}{ negative } & \multirow[t]{2}{*}{514 nm, LED } & $\sim 7.5$ \\
\hline violet LED & & & $\sim 13$ \\
\hline 488 nm Laser & \multirow[t]{2}{*}{ negative } & \multirow[t]{2}{*}{$488 \mathrm{~nm}$, laser } & $\sim 3.1-7.4$ \\
\hline $401 \mathrm{~nm}$ Laser & & & $\sim 2.9-6.7$ \\
\hline $514 \mathrm{~nm}$ Laser & \multirow[t]{3}{*}{ decoupled } & \multirow[t]{3}{*}{514 , laser } & $\sim 3.1$ \\
\hline $401 \mathrm{~nm}$ Laser & & & $\sim 3.1$ \\
\hline 375 nm Laser & & & $\sim 0.2$ \\
\hline
\end{tabular}

Table 3. Approximate values for surface power density of the light sources used for RSFP switching on both setups.

\subsection{Algorithms}

The SOFI algorithm used for all calculations in this work was written by Simon Christoph Stein, University of Göttingen.

The script for analysis of the RSFP photoswitching was based on a script kindly provided by Azat Sharipov, group of Prof. Dr. Theo Lasser, Laboratoire d'Optique Biomédicale, École polytechnique fédérale de Lausanne, Switzerland.

STORM data were analyzed using rapidSTORM 66. 


\section{Supplementary data}

\subsection{Determination of photoswitching}

In contrast to rsEGFP(N205S) and Dreiklang, the photoswitching of rsFastLime, Dronpa(M159T), rsEGFP, and rsEGFP2 frequently deviated from the expected dependence on the irradiation intensities.

\subsection{1 rsEGFP}

$\tau_{\text {off }}$ of rsEGFP (Fig. 42) decreased with increasing intensity of the off-switching light under all irradiation conditions. Furthermore, the $\tau_{\text {off }}$ curves were almost

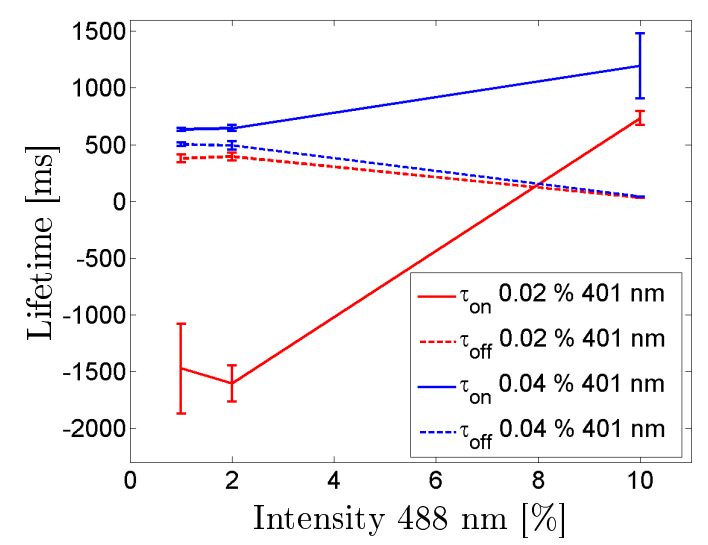

(a) 488 and $401 \mathrm{~nm}$ LED

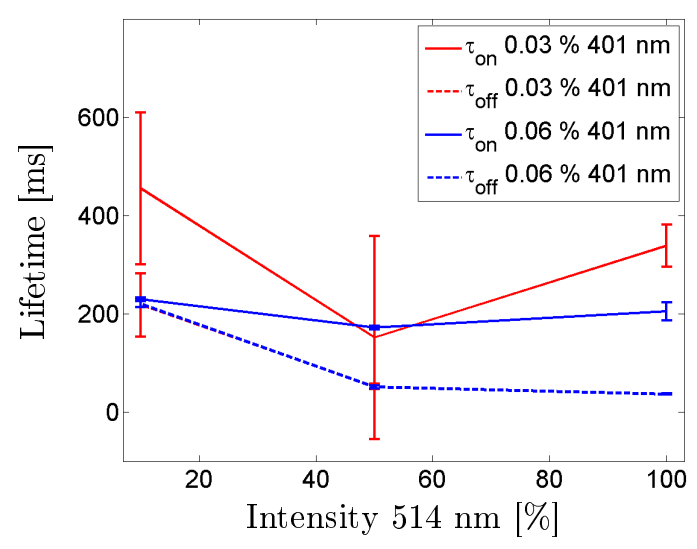

(b) 514 and $401 \mathrm{~nm}$ LED

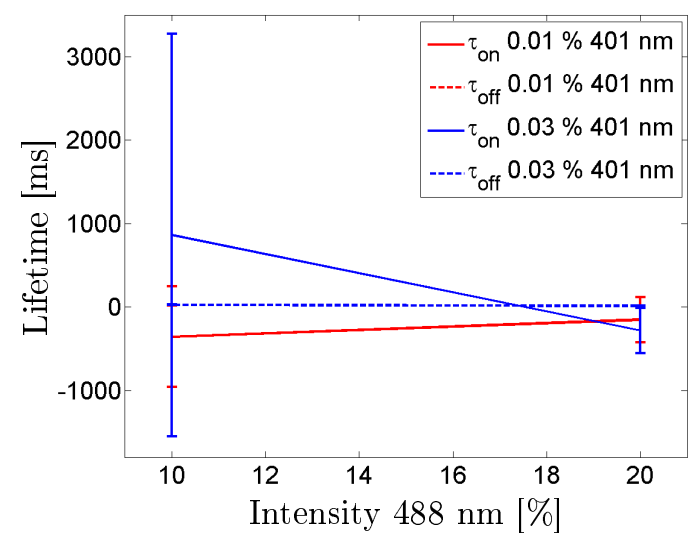

(c) 488 and $401 \mathrm{~nm}$ laser

Figure 42. rsEGFP photoswitching kinetics under different irradiation conditions. 
identical for different intensities of $375 \mathrm{~nm}$, thus showing all estimated dependencies. This was not the case for $\tau_{o n}$, where the values were not independent of the offswitching intensity, contrary to expectations. Moreover, $\tau_{\text {on }}$ values were frequently negative. This is physically impossible and indicates large deviations from the model for chromophore switching that the analysis was based on.

\subsection{2 rsEGFP2}

rsEGFP2 showed interesting photoswitching under 488 and $401 \mathrm{~nm}$ LED irradiation (Fig. $43 \mathrm{a}$ and Fig. $43 \mathrm{~b}$ ). While the $\tau_{\text {off }}$ values at the lower $401 \mathrm{~nm}$ intensity $(0.04$

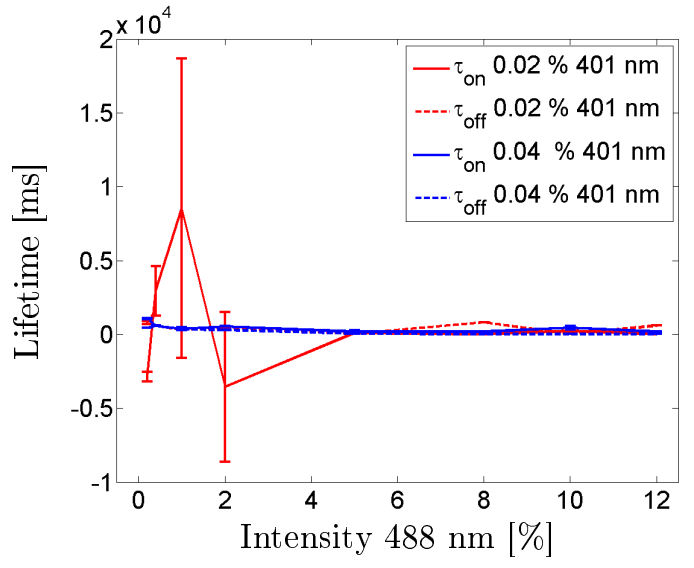

(a) 488 and $401 \mathrm{~nm}$ LED

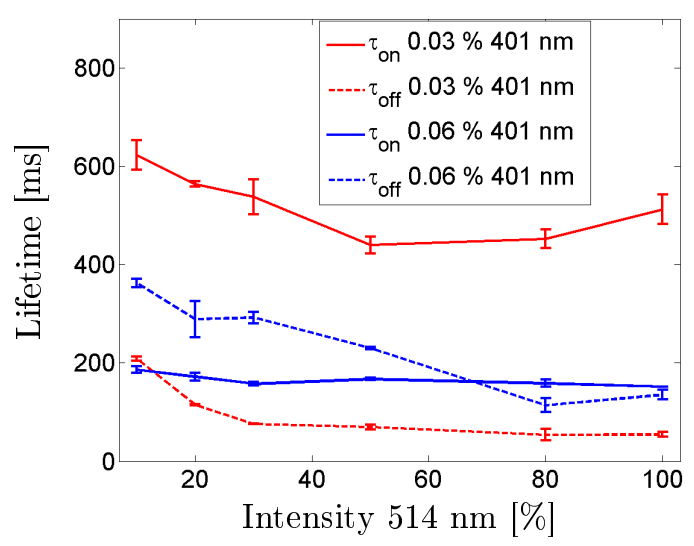

(c) 514 and $401 \mathrm{~nm}$ LED

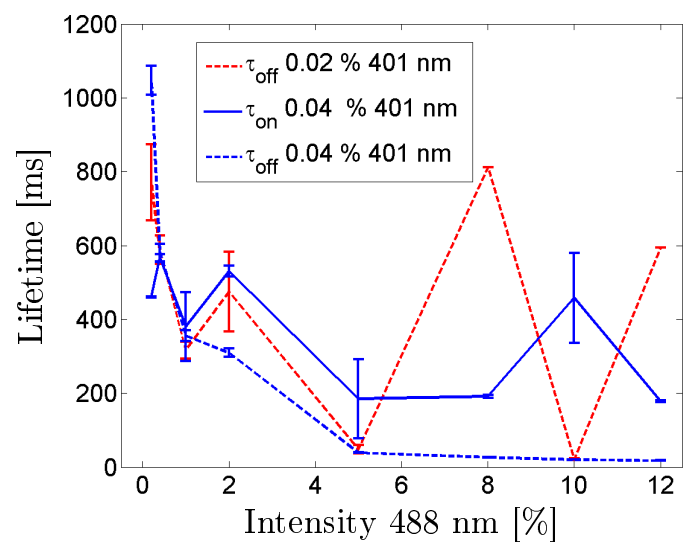

(b) 488 and $401 \mathrm{~nm}$ LED (without $\tau_{\text {on }}$ of $0.02 \% 401 \mathrm{~nm}$ )

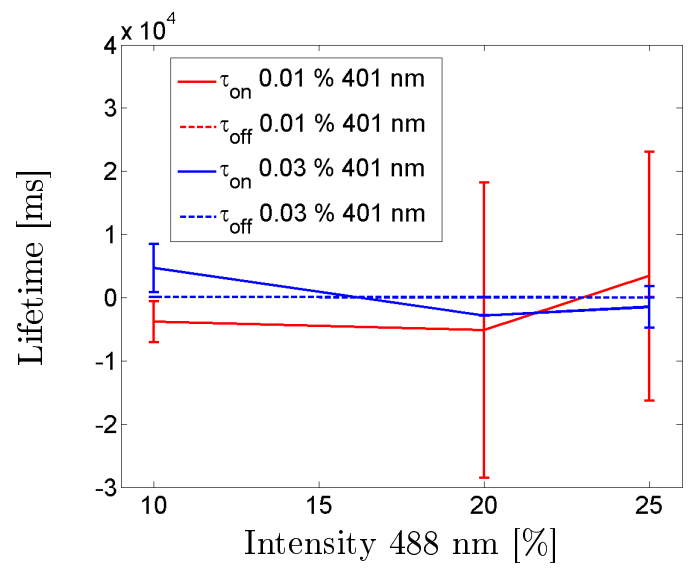

(d) 488 and $401 \mathrm{~nm}$ laser

Figure 43. rsEGFP2 photoswitching under different irradiation conditions. 
$\%$ ) were roughly as expected, $\tau_{\text {off }}$ at the higher $401 \mathrm{~nm}$ intensity and $\tau_{\text {on }}$ at all intensities lacked any clear dependencies. Under 514 and $401 \mathrm{~nm}$ LED irradiation (Fig. 43r), the dependencies of the lifetimes on the irradiation intensities were closer to the estimations. A notable exception was the large difference between the values of $\tau_{\text {off }}$ for both $401 \mathrm{~nm}$ intensities. Under laser irradiation (Fig. $43 \mathrm{~d}$ ), both $\tau_{\text {off }}$ curves had similar values and showed the expected decrease (from 108 to $40 \mathrm{~ms}$ for $0.01 \%$ and from 113 to $39 \mathrm{~ms}$ for $0.03 \% 401 \mathrm{~nm}$ intensity). The values of $\tau_{\text {on }}$, on the other hand, were mostly negative and had large standard deviations.

\subsection{3 rsFastLime}

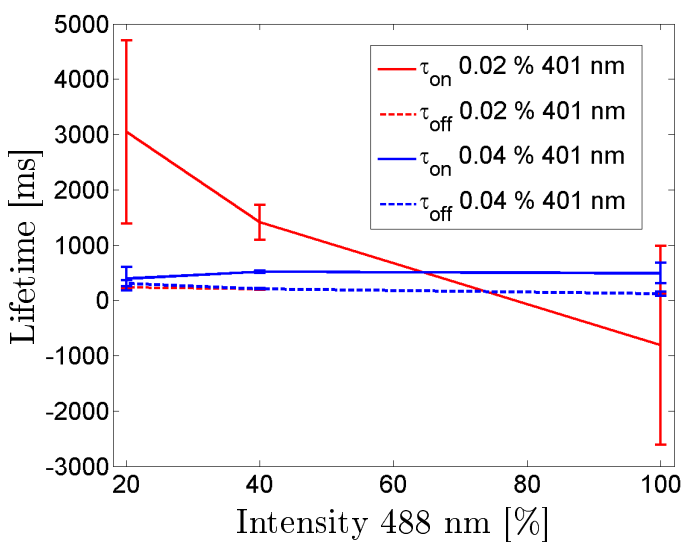

(a) 488 and $401 \mathrm{~nm}$ LED

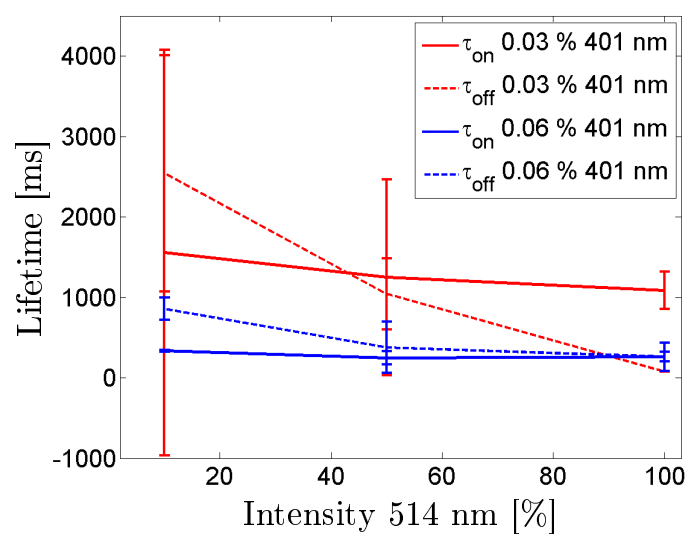

(b) 514 and $401 \mathrm{~nm}$ LED

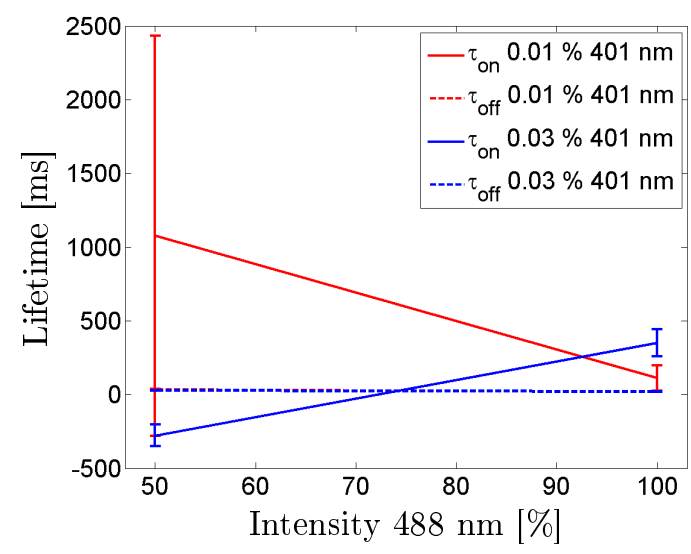

(c) 488 and $401 \mathrm{~nm}$ laser

Figure 44. rsFastLime photoswitching under different irradiation conditions. 
For all studied irradiation conditions, $\tau_{\text {off }}$ of rsFastLime showed the estimated dependence on the irradiation intensities (Fig. 44). The only exception was the difference of the curves for different intensities of $401 \mathrm{~nm}$ irradiation for 514 and $401 \mathrm{~nm}$ LED irradiation (Fig. 44 b). $\tau_{\text {on }}$ values were largely negative with large standard deviations, similar to the results obtained for rsEGFP and rsEGFP2 photoswitching.

\subsubsection{Dronpa(M159T)}

$\tau_{\text {off }}$ for Dronpa(M159T) showed the estimated dependence on 488 and $401 \mathrm{~nm}$ LED as well as laser irradiation (Fig. 45). For most intensities, however, $\tau_{\text {on }}$ was not constant with respect to $488 \mathrm{~nm}$ irradiation, but decreased with increasing intensity.

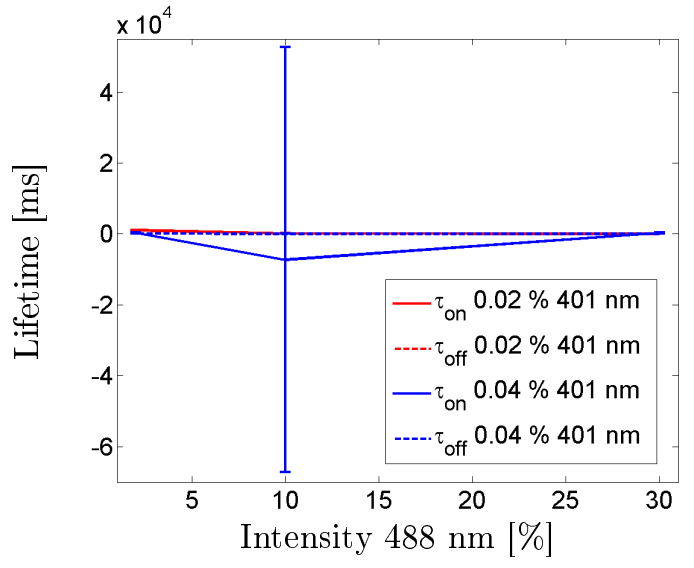

(a) 488 and $401 \mathrm{~nm}$ LED

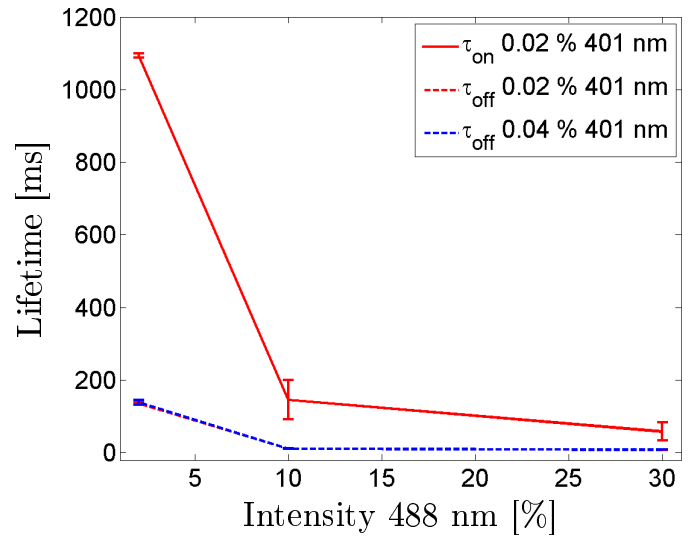

(b) 488 and $401 \mathrm{~nm}$ LED (without $\tau_{\text {on }}$ of $0.04 \% 401 \mathrm{~nm}$ )

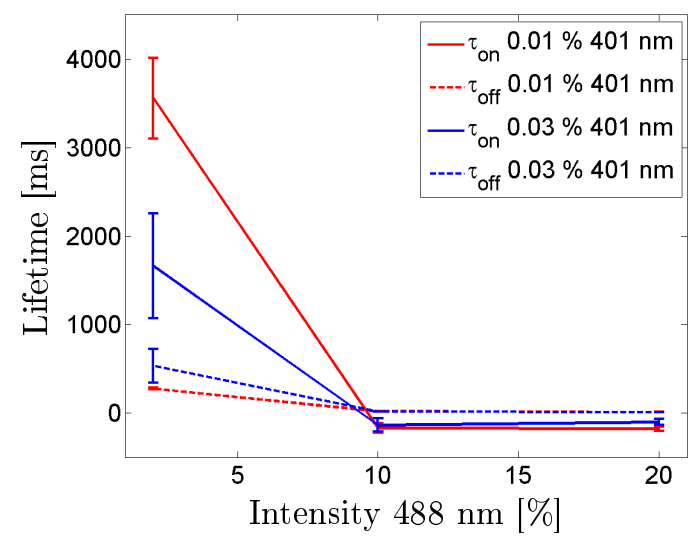

(c) 488 and $401 \mathrm{~nm}$ laser

Figure 45. Dronpa(M159T) photoswitching under different irradiation conditions. 


\subsubsection{Discussion}

The measured dependencies of the negative photoswitching of rsEGFP, rsEGFP2, rsFastLime, and Dronpa(M159T) on irradiation intensities deviated considerably from the estimations. This indicated additional effects that were not taken into account by the model. Although SOFI is flexible with regard to the intensity fluctuations of the labels, precise control of the photoswitching is favorable. In order to control the photoswitching, reliable measurements of the on- and off-times are necessary. If the perceived lifetimes of proteins deviate too much from the actual lifetimes, adjusting the on- and off-times to desired values is not possible. Proteins whose photoswitching severely deviates from the model are therefore not suitable as labels for SOFI.

The frequently observed decrease of $\tau_{\text {off }}$ with increasing intensity of $488 \mathrm{~nm}$ irradiation suggests extra on-switching, and differing $\tau_{\text {on }}$ curves for different intensities of $401 \mathrm{~nm}$ irradiation indicate thermal relaxation. Effects that impair the accuracy of the fits include bleaching as well as poor SNR, to which an ensemble of emitters with very long off-times is especially prone.

For proteins with negative switching, $\tau_{\text {rise }}$ is determined under irradiation with both on- and off-switching light, as the off-switching light is necessary to elicit fluorescence. As a result, $\tau_{\text {rise }}$ measures the competing processes of on- and off-switching. The lifetime of the off-state $\tau_{\text {on }}$ is calculated from $\tau_{\text {off }}=\tau_{\text {fall }}$ and $\tau_{\text {rise }}$ according to $1 / \tau_{\text {on }}=1 / \tau_{\text {rise }}-1 / \tau_{\text {fall }}$ (Eq. 15). The corresponding rate constants $k_{\text {off }}$ and $k_{\text {on }}$ then depend only on $488 \mathrm{~nm}$ and $401 \mathrm{~nm}$ irradiation, respectively. However, due to additional on-switching mediated by $488 \mathrm{~nm}$ irradiation, the rate constants measured in the experiments $\left(k_{o f f_{-} a p p}\right.$ and $\left.k_{\text {on_app }}\right)$ consist of the actual constants $\left(k_{o f f}\right.$ and $\left.k_{o n}\right)$ and an additional rate constant $k_{x}$, leading to

$$
k_{o f f_{-} a p p}=k_{o f f}-k_{x} \quad(17) \quad \text { and } \quad k_{\text {on_app }}=k_{\text {on }}+k_{x} .
$$

The efficiency of the on-switching is higher for shorter wavelengths. Consequently, the effect was strongest for $488 \mathrm{~nm}$ LED irradiation, which contained wavelengths as short as $475 \mathrm{~nm}$, and weak for $514 \mathrm{~nm}$ LED and $488 \mathrm{~nm}$ laser irradiation. Furthermore, the extra transitions to the on-state had the strongest effect on the $\tau_{\text {on }}$ curves for low $401 \mathrm{~nm}$ intensities, because there the ratio of additional on-switching mediated by the off-switching light (488 or $514 \mathrm{~nm}$ ) to the desired on-switching mediated by $401 \mathrm{~nm}$ irradiation is the largest. The effect of the additional on-switching on $\tau_{\text {off }}$ is not obvious from the plots, as $\tau_{\text {off }}$ depends on the $488 \mathrm{~nm}$ intensity to begin with. 


\section{Appendix}

\section{Bibliography}

[1] J. R. Lakowicz, Principles of Fluorescence Spectroscopy, Springer Science + Business Media, Third edition (2006).

[2] B. Amos, G. McConnell, T. Wilson, "2.2 Confocal Microscopy", In Edward H. Egelman, editor, Comprehensive Biophysics, pp. 3 - 23. Elsevier Amsterdam (2012).

[3] T. Tanaami, S. Otsuki, N. Tomosada, Y. Kosugi, M. Shimizu, H. Ishida, "Highspeed 1-frame/ms scanning confocal microscope with a microlens and Nipkow disks" Appl. Opt. (2002) 41(22), 4704-4708.

[4] A. Nakano, "Spinning-disk confocal microscopy - A cutting-edge tool for imaging of membrane traffic" Cell Struct. Funct. (2002) 27(5), 349-355.

[5] E. Abbe, "Beiträge zur Theorie des Mikroskops und der mikroskopischen Wahrnehmung" Archiv für mikroskopische Anatomie (1873) 9(1), 456-468.

[6] S. W. Hell, J. Wichmann, "Breaking the diffraction resolution limit by stimulated emission: stimulated-emission-depletion fluorescence microscopy" Opt. Lett. (1994) 19(11), 780-782.

[7] D. Wildanger, B. R. Patton, H. Schill, L. Marseglia, J. P. Hadden, S. Knauer, A. Schönle, J. G. Rarity, J. L. O’Brien, S. W. Hell, J. M. Smith, "Solid Immersion Facilitates Fluorescence Microscopy with Nanometer Resolution and SubÅngström Emitter Localization" Adv. Mater. (2012) 24(44), OP309-OP313.

[8] M. Hofmann, C. Eggeling, S. Jakobs, S. W. Hell, "Breaking the diffraction barrier in fluorescence microscopy at low light intensities by using reversibly photoswitchable proteins" Proc. Natl. Acad. Sci. USA (2005) 102(49), 1756517569.

[9] M. G. Gustafsson, "Surpassing the lateral resolution limit by a factor of two using structured illumination microscopy" J. Microsc. (2000) 198(Pt 2), 82-87. 
[10] C. B. Müller, J. Enderlein, "Image Scanning Microscopy" Phys. Rev. Lett. (2010) 104(19).

[11] O. Schulz, C. Pieper, M. Clever, J. Pfaff, A. Ruhlandt, R. H. Kehlenbach, F. S. Wouters, J. Großhans, G. Bunt, J. Enderlein, "Resolution doubling in fluorescence microscopy with confocal spinning-disk image scanning microscopy" Proc. Natl. Acad. Sci. USA (2013) 110(52), 21000-21005.

[12] M. J. Rust, M. Bates, Xiaowei Zhuang, "Sub-diffraction-limit imaging by stochastic optical reconstruction microscopy (STORM)" Nat. Methods (2006) 3(10), 793-796.

[13] E. Betzig, G. H. Patterson, R. Sougrat, O. W. Lindwasser, S. Olenych, J. S. Bonifacino, M. W. Davidson, J. Lippincott-Schwartz, H. F. Hess, "Imaging Intracellular Fluorescent Proteins at Nanometer Resolution" Science (2006) 313(5793), 1642-1645.

[14] S. T. Hess, T. P.K. Girirajan, M. D. Mason, "Ultra-High Resolution Imaging by Fluorescence Photoactivation Localization Microscopy" Biophys. J. (2006) 91(11), 4258-4272.

[15] M. Heilemann, S. van de Linde, M. Schüttpelz, R. Kasper, B. Seefeldt, A. Mukherjee, P. Tinnefeld, M. Sauer, "Fluoreszenzmikroskopie unterhalb der optischen Auflösungsgrenze mit konventionellen Fluoreszenzsonden" Angew.Chem. (2008) 120, 6266-6271.

[16] T. Dertinger, R. Colyer, G. Iyer, S. Weiss, J. Enderlein, "Fast, background-free, 3D super-resolution optical fluctuation imaging (SOFI)" Proc. Natl. Acad. Sci. USA (2009) 106(52), 22287-92.

[17] T. Dertinger, R. Colyer, R. Vogel, J. Enderlein, S. Weiss, "Achieving increased resolution and more pixels with Superresolution Optical Fluctuation Imaging (SOFI)" Opt. Express (2010) 18(18), 18875-18885.

[18] W. Gerstner, W. M. Kistler, Spiking Neuron Models: Single Neurons, Populations, Plasticity, Cambridge University Press (2002).

[19] R. S. Swenson, Review of Clinical and Functional Neuroscience, Dartmouth Medical School (2006). 
[20] G. L. Collingridge, J. T. Isaac, Y. T. Wang, "Receptor trafficking and synaptic plasticity" Nat. Rev. Neurosci. (2004) 5(12), 952 - 962.

[21] S. Geissbuehler, C. Dellagiacoma, T. Lasser, "Comparison between SOFI and STORM" Biomed. Opt. Express (2011) 2(3), 408-420.

[22] L. Brus, "Quantum crystallites and nonlinear optics" Appl. Phys. A (1991) 53(6), 465-474.

[23] B. O. Dabbousi, J. Rodriguez-Viejo, F. V. Mikulec, J. R. Heine, H. Mattoussi, R. Ober, K. F. Jensen, M. G. Bawendi, "(CdSe)ZnS Core-Shell Quantum Dots: Synthesis and Characterization of a Size Series of Highly Luminescent Nanocrystallites" J. Phys. Chem. B (1997) 101(46), 9463-9475.

[24] T. Dertinger, J. Xu, O. Naini, R. Vogel, S. Weiss, "SOFI-based 3D superresolution sectioning with a widefield microscope" Opt. Nanoscopy (2012) 1(1), 2 .

[25] A. Huss, O. Ramírez, F. Santibáñez, A. Couve, S. Härtel, J. Enderlein, "SOFI of GABAB neurotransmitter receptors in hippocampal neurons elucidates intracellular receptor trafficking and assembly" Proc. SPIE (2013) 8590, 85900N$85900 \mathrm{~N}-7$.

[26] M. E. Gallina, J. Xu, T. Dertinger, A. Aizer, Y. Shav-Tal, S. Weiss, "Resolving the spatial relationship between intracellular components by dual color super resolution optical fluctuations imaging (SOFI)" Opt. Nanoscopy (2013) 2(1), 2.

[27] S. F. Lee, M. A. Osborne, "Brightening, Blinking, Bluing and Bleaching in the Life of a Quantum Dot: Friend or Foe?" ChemPhysChem (2009) 10(13), 2174-2191.

[28] K. T. Shimizu, R. G. Neuhauser, C. A. Leatherdale, S. A. Empedocles, W. K. Woo, M. G. Bawendi, "Blinking statistics in single semiconductor nanocrystal quantum dots" Phys. Rev. B (2001) 63(20), 205316:1-5.

[29] M. Kuno, D. P. Fromm, H. F. Hamann, A. Gallagher, D. J. Nesbitt, "Nonexponential "blinking" kinetics of single CdSe quantum dots: A universal power law behavior" J. Chem. Phys. (2000) 112(7), 3117-3120.

[30] S. J. Thuault, J. T. Brown, S. A. Sheardown, S. Jourdain, B. Fairfax, J. P. Spencer, S. Restituito, J. H.L. Nation, S. Topps, A. D. Medhurst, A. D. Randall, 
A. Couve, S. J. Moss, G. L. Collingridge, Me. N. Pangalos, C. H. Davies, A. R. Calver, "The $\mathrm{GABA}_{\mathrm{B} 2}$ subunit is critical for the trafficking and function of nativeGABA $A_{B}$ receptors" Biochem. Pharmacol. (2004) 68(8), 1655 - 1666.

[31] O. A. Ramírez, R. L. Vidal, J. A. Tello, K. J. Vargas, S. Kindler, S. Härtel, A. Couve, "Dendritic Assembly of Heteromeric $\gamma$-Aminobutyric Acid Type B Receptor Subunits in Hippocampal Neurons" J. Biol. Chem. (2009) 284(19), 13077-13085.

[32] K. J. Seymour-Laurent, M. E. Barish, "Inositol 1,4,5-trisphosphate and ryanodine receptor distributions and patterns of acetylcholine-induced and caffeineinduced calcium-release in cultured mouse hippocampal-neurons" J. Neurosci. (1995) 15(4), 2592-2608.

[33] J. S. Fitzpatrick, A. M. Hagenston, D. N. Hertle, K. E. Gipson, L. BertettoD’Angelo, M. F. Yeckel, "Inositol-1,4,5-trisphosphate receptor-mediated Ca2+ waves in pyramidal neuron dendrites propagate through hot spots and cold spots" J. Physiol. (2009) 587(7), 1439-1459.

[34] I. I. Serysheva, "Toward a high-resolution structure of IP3R channel" Cell Calcium (2014) 56(3), 125 - 132.

[35] F. Van Petegem, "Ryanodine Receptors: Structure and Function" J. Biol. Chem. (2012) 287(38), 31624-31632.

[36] T. K. Kim, J. H. Eberwine, "Mammalian cell transfection: the present and the future" Anal. Bioanal. Chem. (2010) 397(8), 3173-3178.

[37] D. M. Chudakov, M. V. Matz, S. Lukyanov, K. A. Lukyanov, "Fluorescent Proteins and Their Applications in Imaging Living Cells and Tissues" Physiol. Rev. (2010) 90(3), 1103-1163.

[38] Alexey A. Pakhomov, Vladimir I. Martynov, " $\{$ GFP $\}$ Family: Structural Insights into Spectral Tuning" Chemistry \& Biology (2008) 15(8), 755 - 764.

[39] O. Shimomura, F. H. Johnson, Y. Saiga, "Extraction, Purification and Properties of Aequorin, a Bioluminescent Protein from the Luminous Hydromedusan, Aequorea" J. Cell. Physio. (1962) 59(3), 223-239.

[40] D. C. Prasher, V. K. Eckenrode, W. W. Ward, F. G. Prendergast, M. J. Cormier, "Primary structure of the Aequorea victoria green-fluorescent protein" Gene (1992) 111(2), $229-233$. 
[41] M. Chalfie, Y. Tu, G. Euskirchen, W. W. Ward, D. C. Prasher, "Green fluorescent protein as a marker for gene expression" Science (1994) 263(5148), 802-805.

[42] G. H. Patterson, S. M. Knobel, W. D. Sharif, S. R. Kain, D. W. Piston, "Use of the green fluorescent protein and its mutants in quantitative fluorescence microscopy" Biophys. J. (1997) 73(5), 2782-2790.

[43] R. E. Campbell, O. Tour, A. E. Palmer, P. A. Steinbach, G. S. Baird, D. A. Zacharias, R. Y. Tsien, "A monomeric red fluorescent protein" Proc. Natl. Acad. Sci. USA (2002) 99(12), 7877-7882.

[44] N. C. Shaner, R. E. Campbell, P. A. Steinbach, B. N. G. Giepmans, A. E. Palmer, R. Y. Tsien, "Improved monomeric red, orange and yellow fluorescent proteins derived from Discosoma sp red fluorescent protein" Nat. Biotechnol. (2004) 22(12), 1567-1572.

[45] J. Wiedenmann, S. Ivanchenko, F. Oswald, F. Schmitt, C. Rocker, A. Salih, K. D. Spindler, G. U. Nienhaus, "EosFP, a fluorescent marker protein with UV-inducible green-to-red fluorescence conversion" Proc. Natl. Acad. Sci. USA (2004) 101(45), 15905-15910.

[46] S. Wang, J. R. Moffitt, G. T. Dempsey, X. S. Xie, X. Zhuang, "Characterization and development of photoactivatable fluorescent proteins for single-moleculebased superresolution imaging" Proc. Natl. Acad. Sci. USA (2014) 111(23), 8452-8457.

[47] R. M. Dickson, A. B. Cubitt, R. Y. Tsien, W. E. Moerner, "On/off blinking and switching behaviour of single molecules of green fluorescent protein" Nature (1997) 388(6640), 355-358.

[48] D. M. Chudakov, V. V. Belousov, A. G. Zaraisky, Vl. V. Novoselov, D. B. Staroverov, D. B. Zorov, S. Lukyanov, K. A. Lukyanov, "Kindling fluorescent proteins for precise in vivo photolabeling" Nat. Biotechnol. (2003) 21(2), 191194.

[49] R. Ando, H. Mizunoi, A. Miyawaki, "Regulated Fast Nucleocytoplasmic Shuttling Observed by Reversible Protein Highlighting" Science (2004) 306(5700), $1370-1373$. 
[50] D. Bourgeois, V. Adam, "Reversible photoswitching in fluorescent proteins: A mechanistic view" IUBMB Life (2012) 64(6), 482-491.

[51] X. X. Zhou, M. Z. Lin, "Photoswitchable fluorescent proteins: ten years of colorful chemistry and exciting applications" Curr. Opin. Chem. Biol. (2013) $\mathbf{1 7}(4), 682-690$.

[52] T. Brakemann, A. C. Stiel, G. Weber, M. Andresen, I. Testa, M. Leutenegger T. Grotjohann, U. Plessmann, H. Urlaub, C. Eggeling, M. C. Wahl, S. W. Hell, S. Jakobs, "A reversibly photoswitchable GFP-like protein with fluorescence excitation decoupled from switching" Nat. Biotechnol. (2011) 29(10), 942-950.

[53] T. Grotjohann, I. Testa, M. Leutenegger, H. Bock, N. T. Urban, F. LavoieCardinal, K. I. Willig, C. Eggeling, S. Jakobs, S. W. Hell, "Diffractionunlimited all-optical imaging and writing with a photochromic GFP" Nature (2011) 478(7368), 204-208.

[54] M. Andresen, M. C. Wahl, A. C. Stiel, F. Gräter, L. V. Schäfer, S. Trowitzsch, G. Weber, C. Eggeling, H. Grubmüller, S. W. Hell, S. Jakobs, "Structure and mechanism of the reversible photoswitch of a fluorescent protein" Proc. Natl. Acad. Sci. USA (2005) 102(37), 13070-13074.

[55] M. Andresen, A.C. Stiel, S. Trowitzsch, G. Weber, C. Eggeling, M. C. Wahl, S. W. Hell, S. Jakobs, "Structural basis for reversible photoswitching in Dronpa" Proc. Natl. Acad. Sci. USA (2007) 104(32), 13005-13009.

[56] A. C. Stiel, S. Trowitzsch, G. Weber, M. Andresen, C. Eggeling, S. W. Hell, S. Jakobs, M. C. Wahl, "1.8 A bright-state structure of the reversibly switchable fluorescent protein Dronpa guides the generation of fast switching variants" Biochem. J. (2007) 402(1), 35-42.

[57] T. Grotjohann, I. Testa, M. Reuss, T. Brakemann, C. Eggeling, S. W. Hell, S. Jakobs, "rsEGFP2 enables fast RESOLFT nanoscopy of living cells" eLife (2012) 1 .

[58] A. Chmyrov, J. Keller, T. Grotjohann, M. Ratz, E. d'Este, S. Jakobs, C. Eggeling, S. W. Hell, "Nanoscopy with more than 100,000 'doughnuts", Nat. Methods (2013) 10(8), 737-740. 
[59] N. A. Jensen, J. G. Danzl, K. I. Willig, F. Lavoie-Cardinal, T. Brakemann, S. W. Hell, S. Jakobs, "Coordinate-Targeted and Coordinate-Stochastic SuperResolution Microscopy with the Reversibly Switchable Fluorescent Protein Dreiklang" ChemPhysChem (2014) 15(4, SI), 756-762.

[60] P. Dedecker, G. C. H. Mo, T. Dertinger, J. Zhang, "Widely accessible method for superresolution fluorescence imaging of living systems" Proc. Natl. Acad. Sci. USA (2012).

[61] B. Moeyaert, N. Nguyen Bich, E. De Zitter, S. Rocha, K. Clays, H. Mizuno, L. van Meervelt, J. Hofkens, P. Dedecker, "Green-to-Red Photoconvertible Dronpa Mutant for Multimodal Super-resolution Fluorescence Microscopy" ACS Nano (2014) 8(2), 1664-1673.

[62] S. van de Linde, A. Löschberger, T. Klein, M. Heidbreder, S. Wolter, M. Heilemann, M. Sauer, "Direct stochastic optical reconstruction microscopy with standard fluorescent probes" Nat. Protoc. (2011) 6(7), 991-1009.

[63] G. T. Dempsey, J. C. Vaughan, K. H. Chen, M. Bates, X. Zhuang, "Evaluation of fluorophores for optimal performance in localization-based super-resolution imaging" Nat. Methods (2011) 8(12), 1027-1036.

[64] T. Dertinger, M. Heilemann, R. Vogel, M. Sauer, S. Weiss, "Superresolution Optical Fluctuation Imaging with Organic Dyes" Angew. Chem. Int. Ed. (2010) 49(49), 9441-9443.

[65] S. Geissbuehler, N. Bocchio, C. Dellagiacoma, C. Berclaz, M. Leutenegger, T. Lasser, "Mapping molecular statistics with balanced super-resolution optical fluctuation imaging (bSOFI)" Opt. Nanoscopy (2012) 1(1), 4.

[66] S. Wolter, M. Schüttpelz, M. Tscherepanow, S. Van de Linde, M. Heilemann, M. Sauer, "Real-time computation of subdiffraction-resolution fluorescence images" J. Microsc. (Oxf) (2010) 237(1), 12-22.

[67] M. H. P. Kole, G. J. Stuart, "Signal Processing in the Axon Initial Segment" Neuron (2012) 73(2), $235-247$.

[68] S. A. Buffington, M. N. Rasband, "The axon initial segment in nervous system disease and injury" Eur. J. Neurosci. (2011) 34(10), 1609-1619. 
[69] A. J. Baines, "The spectrin-ankyrin-4.1-adducin membrane skeleton: adapting eukaryotic cells to the demands of animal life" Protoplasma (2010) 244(1-4), 99-131.

[70] K. Xu, G. Zhong, X. Zhuang, "Actin, Spectrin, and Associated Proteins Form a Periodic Cytoskeletal Structure in Axons" Science (2013) 339(6118), 452-456.

[71] V. Bennett, A. J. Baines, "Spectrin and ankyrin-based pathways: metazoan inventions for integrating cells into tissues" Physiol. Rev. (2001) 81(3), 13531392.

[72] K. Goslin, G. Banker, editors, Culturing Nerve Cells, pp. 251-281. Massachusetts Institute of Technology, Cambridge, MA (1991).

[73] M. Jiang, , G. Chen, "High Ca2+-phosphate transfection efficiency in lowdensity neuronal cultures" Nat. Protoc. (2006) 1(2), 695-700.

[74] J. Chuang, T. Yeh, F. Bollati, C. Conde, F. Canavosio, A. Caceres, C. Sung, "The Dynein Light Chain Tctex-1 Has a Dynein-Independent Role in Actin Remodeling during Neurite Outgrowth" Dev. Cell (2005) 9(1).

[75] J. Morin-Leisk, S. G. Saini, X. Meng, A. M. Makhov, P. Zhang, T. H. Lee, "An intramolecular salt bridge drives the soluble domain of GTP-bound atlastin into the postfusion conformation" J. Cell. Biol. (2011) 195(4), 605-615.

[76] G. M. J. Beaudoin III, S. Lee, D. Singh, Y. Ng Y. Yuan, L. F. Reichardt, J. Arikkath, "Culturing pyramidal neurons from the early postnatal mouse hippocampus and cortex" Nat. Protoc. (2012) 7(9), 1741-1754.

[77] N. Goeden, A. Bonnin, "Ex vivo perfusion of mid-to-late-gestation mouse placenta for maternal-fetal interaction studies during pregnancy" Nat. Protoc. (2013) 8(1), 66-74.

[78] N. Pfennig, S Wagener, "An improved method of preparing wet mounts for photomicrographs of microorganisms" J. Mircrobiol. Meth. (1986) 4(5-6), 303306. 


\section{List of Figures}

$1 \quad$ Jablonski diagram $\ldots \ldots \ldots \ldots \ldots \ldots$

2 Widefield fluorescence image of a neuron . . . . . . . . . . . 5

$3 \quad$ PSF of single QDots . . . . . . . . . . . . . . . 7

$4 \quad$ SOFI schematic . . . . . . . . . . . . . . . . . . . . 13

5 Widefield, SOFI and Fourier-reweighted SOFI OTF and PSF . . . . 18

$6 \quad$ Evaluation of exposure time for QDot SOFT . . . . . . . . . . . 25

7 Evaluation of number of frames for QDot SOFT . . . . . . . . . . 25

$8 \quad$ Axial drift, widefield microscope equipped with LEDs . . . . . . . . . 26

$9 \quad$ Lateral drift, widefield microscope equipped with LEDs. . . . . . . . . 27

10 Comparison of widefield-, basic-SOFI-, and FR-SOFI-PSF of single

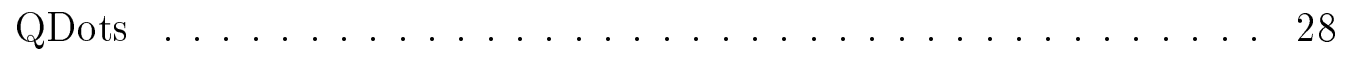

11 Comparison of different stages of SOFI processing on an image of QDots 30

12 Trafficking of $\mathrm{GABA}_{\mathrm{B}}$ receptor subunits $\ldots \ldots \ldots \ldots \ldots$. . . . . . . 32

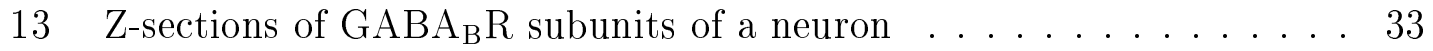

14 Complete z-stack of $\mathrm{GABA}_{\mathrm{B}} \mathrm{R}$ subunits of a neuron . . . . . . . . . 34

15 Comparison of SOFI and confocal microscopy . . . . . . . . . . 36

$16 \quad \mathrm{ER} \mathrm{IP}_{3}$ receptors and ryanodine receptors of a neuron $\ldots . . . . . .38$

$17 \quad \mathrm{IP}_{3}$ and ryanodine receptors of a neuron $\ldots \ldots \ldots \ldots \ldots$

18 3D SOFI projection of ryanodine receptors . . . . . . . . . . . . . 40

19 Illustration of negative and decoupled switching . . . . . . . . . . . 43

20 Time trace of $\operatorname{rsEGFP}(\mathrm{N} 205 \mathrm{~S})$. . . . . . . . . . . . . . . 47

21 Time trace of Dreiklang . . . . . . . . . . . . . . . . . . . . . . 48

22 Time traces of rsEGFP(N205S) at different irradiation intensity combinations . . . . . . . . . . . . . . . 5 50

23 Estimated dependence of switching kinetics on laser intensity . . . . . 51

24 Photoswitching kinetics of rsEGFP(N205S) under different irradiation conditions . . . . . . . . . . . . . . . 53

25 Photoswitching kinetics of Dreiklang . . . . . . . . . . . . . . 55

26 Widefield and basic SOFI image of rsEGFP(N205S)-labeled vimentin 59

27 Widefield and basic SOFI image of rsEGFP(N205S)-labeled vimentin 59

28 Widefield, basic SOFI and deconvolved SOFI image of Dreiklanglabeled vimentin . . . . . . . . . . . . . . . . 6 60

29 Widefield and deconvolved SOFI image of Dreiklang-labeled vimentin 61

30 Schematic of pulsed excitation . . . . . . . . . . . . . . 62 
31 SOFI of Dreiklang-labeled vimentin with pulsed excitation . . . . . . 63

32 Molecular structure of Alexa Fluor 647 . . . . . . . . . . . . . . . 67

33 Energy levels of organic dye photoswitching . . . . . . . . . . . . . 68

34 Schematic of the AIS organization . . . . . . . . . . . . 70

35 Spectrin with Alexa Fluor 647, STORM optimized . . . . . . . . . . . 72

$36 \quad$ Spectrin with Alexa Fluor 647, STORM optimized, cross section . . . 73

37 Spectrin with Alexa Fluor 647 , SOFI optimized . . . . . . . . . . . . 74

38 Spectrin with Alexa Fluor 647, SOFI optimized, zoom-ins . . . . . . . 75

39 Spectrin with Alexa Fluor 647, SOFI optimized, cross section . . . . . 75

40 Sodium channels with Alexa Fluor $488 \ldots \ldots$. . . . . . . . . . 76

41 Sodium channels with Alexa Fluor 488, cross section . . . . . . . . 77

$42 \quad$ Photoswitching kinetics of rsEGFP $\ldots \ldots \ldots \ldots$. . . . . . . . . 93

43 Photoswitching kinetics of rsEGFP2 $\ldots \ldots \ldots \ldots$. . . . . . . . 94

44 Photoswitching kinetics of rsFastLime . . . . . . . . . . . . . 95

45 Photoswitching kinetics of Dronpa(M159T) . . . . . . . . . . . 96 


\section{List of Tables}

1 Comparison of the PSF FWHM of single QDots for different stages of SOFI processing . . . . . . . . . . . . . . . . . . 29

$2 \quad$ Lifetimes of on- and off-states . . . . . . . . . . . . . . . . . 49

$3 \quad$ Approximate surface power densities . . . . . . . . . . . . . . . . 91 


\section{Acronyms}

AC-SOFI autocorrelation stochastic optical fluctuation imaging

AIS axon initial segment

AP action potential

ATL-2 atlastin-2

BSA bovine serum albumin

DIV days in vitro

dSTORM direct stochastic optical reconstruction microscopy

EGFP enhanced green fluorescent protein

ER endoplasmic reticulum

FP fluorescent protein

FPALM fluorescence photoactivation localization microscopy

FR-SOFI Fourier reweighted stochastic optical fluctuation imaging

fSOFI Fourier stochastic optical fluctuation imaging

FWHM full width at half maximum

GABA $\quad \gamma$-Aminobutyric acid

GABA $_{\mathbf{B}} \quad \gamma$-Aminobutyric acid receptor type $\mathrm{B}$

GABA $_{B}$ R1 $\quad \gamma$-Aminobutyric acid receptor type B subunit 1

$\mathrm{GABA}_{\mathrm{B}} \mathrm{R} 2 \quad \gamma$-Aminobutyric acid receptor type B subunit 2

GFP green fluorescent protein

HOMO highest occupied molecular orbital

IC internal conversion

IF immunofluorescence

$\mathbf{I P}_{\mathbf{3}} \quad$ inositol trisphosphate

$\mathbf{I P}_{\mathbf{3}} \mathbf{R} \mathbf{1} \quad$ inositol trisphosphate receptor type 1

ISC intersystem crossing

LUMO lowest unoccupied molecular orbital

NA numerical aperture

Nav sodium channels

OTF optical transfer function

PA-FP photoactivatable fluorescent protein

PALM photoactivated localization microscopy

PBS phosphate buffered saline

PSF point spread function

QDot Quantum Dot 
QD $_{525} \quad$ Quantum Dot with emission wavelength $525 \mathrm{~nm}$

QD $_{605} \quad$ Quantum Dot with emission wavelength $605 \mathrm{~nm}$

RESOLFT reversible saturable optical fluorescence transitions

RFP red fluorescent protein

rsEGFP reversibly photoswitchable ehanced green fluorescent protein

RSFP reversibly photoswitchable fluorescent protein

RT room temperature

RyR2 ryanodine receptor type 2

SNR signal-to-noise ratio

SOFI stochastic optical fluctuation imaging

STED stimulated-emission-depletion

STORM stochastic optical reconstruction microscopy

XC-SOFI crosscorrelation stochastic optical fluctuation imaging 


\section{Acknowledgments}

My heartfelt thanks go to:

Ich danke:

* Jörg Enderlein, for supervising my thesis, providing me with a multitude of projects, and giving me the opportunity to visit exciting conferences

* The members of my thesis committee, Fred S. Wouters and Stefan W. Hell, for their input and support

* Simon Christoph Stein, for his great work on the computational aspects of SOFI, and Olaf Schulz, for lots of technical assistance

* Melanie Dannemeyer, Elinor Lazarov, and Andreas Neef, for the interesting and enjoyable collaboration on AIS organization

* Nickels Jensen, for all those samples and for sharing his expert knowledge of fluorescent proteins, and Stefan Jakobs

* Omar Ramírez and Steffen Härtel, for our collaboration on SOFI applications and a great week in Santiago de Chile

* Stefan Geissbühler, Azat Sharipov, Marcel Leutenegger, and Theo Lasser for our joint work on SOFI with RSFPs and for welcoming me so warmly to Lausanne

* Sebastian van de Linde, for helping me to get the hang of dSTORM, and Markus Sauer

* The competent and helpful Elke Zech, Sabine Huhnold, Nicole Rehbein und Ursula Hahn-Wörgötter, with special thanks to Elke for expertly handling the paperwork for about a million trips

* The fast and precise people form the workshop and Thomas Geiling for IT support (especially his persistence that time Origin broke down)

* Frauke Bergmann, Antje Erdmann, and especially Tina Trost from the PBCS office, for tirelessly guiding me through the formalities

* All my colleagues, for all the mundane and invaluable help, like hunting for filters or chemicals, telling me that matlab command I was not sure existed, placing orders, and generally sharing their expertise and energy with me 
* All my colleagues again for all the fun, jokes, and the wonderful nights out, and for their support in the more dire times. Special thanks to:

Narain "Dude. Need a hug?" Karedla,

Jan "Wenn du besser wärst, dann könntest du das" Thiart,

Simon Christoph "Soup-er detective" Stein,

Daja "Would some chocolate help?" Ruhlandt,

Jan-Matthias "Also deine Brotdose ist schon toll..." Braun,

Michael "Muldenkipper" Fauth,

Simon "GUUUTEN MOORGEEN!!!" Reich

and Melanie "Kommst heut mit zum Sport?" Dannemeyer

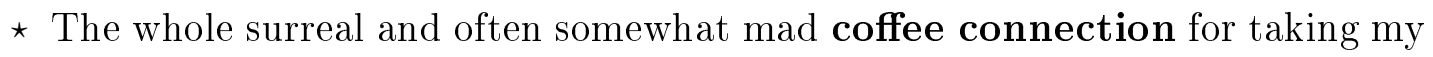
mind completely off science during lunch breaks

* My office mates Hao Cheng, for delicious green tea and pondering cultural peculiarities with me, and Dirk Hähnel, for technical support and the most colorful comparisons

* My former colleagues Kerstin Weiß, Mira Prior, and Phillip Kroehn, the only person I've ever met suffering from lame-pun-Tourette's

* Ancilla Neu and Alexey and Anna Chizhik, for encouraging me to take a leap of faith from chemistry to biophysics, and therefore to plenty of fascinating topics from computational data analysis over neurotoxins and membrane proteins to slime mold

* meinen Eltern Andrea und Rolf Huss und meiner ganzen Familie für ihre Unterstützung

* Florian Siepel, for emotional and practical support, comfort food, and for introducing me to so many power metal bands that the feeling of being on an incredibly important mission might never leave me again

* Natascha Czetto, for her support, keeping me (mostly) sane, and always sharing her cookies with me.

Don't blink. Blink and you're dead. Don't turn your back, don't look away, and don't blink. 